JOURNAL OF THE

AMERICAN MATHEMATICAL SOCIETY

Volume 14, Number 3, Pages 579-622

S 0894-0347(01)00363-0

Article electronically published on March 23, 2001

\title{
CONSTRUCTION OF TAME SUPERCUSPIDAL REPRESENTATIONS
}

\author{
JIU-KANG YU
}

\section{INTRODUCTION}

In this paper, we give a quite general construction of supercuspidal representations of $p$-adic groups. Let $F$ be a non-archimedean local field and let $F^{\mathrm{t}}$ be its maximal tamely ramified extension. Let $G$ be a connected reductive group over $F$. To construct a supercuspidal representation, we start with a triple $\left(\vec{G}, \pi_{0}, \vec{\phi}\right)$, where $\vec{G}=\left(G^{0}, \ldots, G^{d}\right)$ is a tower of algebraic subgroups of $G$,

$$
G^{0} \subsetneq \cdots \subsetneq G^{d}=G
$$

such that $Z\left(G^{0}\right) / Z(G)$ is anisotropic and each $G^{i} \otimes F^{\mathrm{t}}$ is split and is a Levi factor of a parabolic subgroup of $G \otimes F^{\mathrm{t}}, \pi_{0}$ is a supercuspidal representation of $G^{0}(F)$ with depth zero, and $\vec{\phi}=\left(\phi_{0}, \ldots, \phi_{d}\right)$ is such that $\phi_{i}$ is a linear character of $G^{i}(F)$ for each $i$. The main result is

Theorem 0.1. Suppose that $0<\operatorname{depth}\left(\phi_{0}\right)<\cdots<\operatorname{depth}\left(\phi_{d}\right)$ and $\phi_{i}$ is $G^{i+1}$ generic for $0 \leq i \leq d-1$. Then we can construct an irreducible supercuspidal representation $\pi$ of $G(F)$ from the triple $\left(\vec{G}, \pi_{0}, \vec{\phi}\right)$. This supercuspidal representation is compactly induced from an open subgroup which is compact modulo the center of $G(F)$.

The notion of depth is defined by Moy-Prasad [MP2]. The notion of a generic character will be defined in $\$ 9$ When $G=\mathrm{GL}_{n}$ or $G$ is the multiplicative group of a central division algebra of dimension $n^{2}$ with $(n, p)=1$, our generic characters are just the generic characters in $\mathrm{My}$ (where the definition is due to Kutzko). Moreover, in these cases, our construction literally specializes to Howe's construction as formulated in $[\mathrm{My}]$, and it is known that the construction yields all supercuspidal representations ( $[\mathrm{My}],[\mathrm{HM}])$.

Notice that the initial datum in our construction consists of very simple objects: linear characters and supercuspidal representations of depth zero. The latter are well understood by the work of Moy, Prasad, and Morris (see \$3).

By the work of Howe, irreducible supercuspidal representations of $\mathrm{GL}_{n}(F)$ with $(n, p)=1$ are parametrized by certain characters of $E^{*}$ as $E$ varies through

Received by the editors August 30, 1999 and, in revised form, November 13, 2000.

2000 Mathematics Subject Classification. Primary 22E50, 11F70; Secondary 20G25.

Key words and phrases. Supercuspidal representation, Hecke algebra.

The author was supported in part by grant DMS 9801633 from the National Science Foundation. 
extensions over $F$ of degree $n$. Notice that such an $E^{*}$ is a maximal torus of $\mathrm{GL}_{n}(F)$. If $D$ is a central division algebra of dimension $n^{2}$ over $F$ with $(n, p)=1$, irreducible (supercuspidal) representations of $D^{*}$ are parametrized by certain characters of $E^{*}$ as $E$ varies through extensions over $F$ of degree dividing $n$. After the work of Howe, several authors have been attempting to construct supercuspidal representations from characters of a (not necessarily maximal) torus which is compact (modulo center). But in view of the local Langlands conjecture, it is more natural to consider linear characters of the centralizer of such a torus. This is the point of view of our approach.

The adjective "tame" in the title refers to the tamely ramified nature of our construction. Howe called the supercuspidal representations he constructed for $\mathrm{GL}_{n}$ with $(n, p)=1$ "tamely ramified supercuspidal representations". Today it seems more appropriate to reserve "tamely ramified representations" for "depth 0 representations" and it is customary to simply refer to the case considered by Howe as "the tame case". Howe's method actually produces some supercuspidal representations for $\mathrm{GL}_{n}$ for any $n$, but it exhausts all supercuspidal representations only when $(n, p)=1$. The Langlands parameters of supercuspidal representations constructed by Howe's method should be the tame ones, as defined by Koch and Zink [KZ].

Similarly our method produces some supercuspidal representations for every $p$ adic reductive group which splits over $F^{\mathrm{t}}$, and they should correspond to "tame" Langlands parameters. At this time it is not clear how one can define "tameness" in terms of the representations themselves. But it is possible that our method yields all supercuspidal representations that deserve to be called "tame". For reductive groups of a fixed type (i.e. associated to a fixed root datum in the sense of $[\mathrm{Sp}]$ ), all supercuspidal representations of groups of that type should be "tame" when $p$ is large enough. In particular, our method should yield all supercuspidal representations when $p$ is large enough relative to the type of $G$.

Our construction has a very nice inductive structure: from the datum $\left(\vec{G}, \pi_{0}, \vec{\phi}\right)$, we actually get an irreducible supercuspidal representation $\pi_{i}$ on each member $G^{i}$ of the tower $\vec{G}$. Therefore, it is very possible that these representations can be analyzed by using Hecke algebra isomorphisms as done by Howe-Moy [HM] for the case of $\mathrm{GL}_{n}$. The required Hecke algebra isomorphisms are given in a conjectural form in 917 . In fact, the conjecture puts the current work into a much wider perspective: it implies that there is a natural bijection between certain representations of $G^{i}$ and certain representations of $G^{i+1}$, sending $\pi_{i}$ to $\pi_{i+1}$. Moreover, these Hecke algebra isomorphisms can be used to study and classify all irreducible admissible representations, as in $\mathrm{HM}$. This paper is written with this goal in mind.

A well-known folklore conjecture says that every supercuspidal representation $\pi$ of $G$ is compactly induced from some $(K, \rho)$, where $K$ is compact modulo center and $\rho$ is a representation of $K$. Moy, Prasad, and Morris (MP2, Mo1]) have shown that the depth zero representation $\pi_{0}$ is compactly induced from some $\left(K^{0}, \rho_{0}\right)$. As stated in the main theorem, our $\pi=\pi\left(\vec{G}, \pi_{0}, \vec{\phi}\right)$ is compactly induced from certain $(K, \rho)$ constructed out of $\left(K^{0}, \rho_{0}\right)$ and other data. Moreover, the following should be true:

Conjecture 0.2. There is a support-preserving algebra isomorphism

$$
\check{\mathcal{H}}(G(F), \rho) \simeq \check{\mathcal{H}}\left(G^{0}(F), \rho_{0}\right) .
$$


It can be shown that there is a support-preserving vector space isomorphism (cf. Corollary [15.5). Notice that $\check{\mathcal{H}}\left(G^{0}(F), \rho_{0}\right)$ is the Hecke algebra of a type of depth 0 (see [BK2] for the theory of types). Lusztig and Kazhdan-Lusztig (see e.g. [L]) have made very deep studies of such Hecke algebras and their representation theory in many important cases. Morris [Mo4] has calculated generators and relations of such a Hecke algebra. The above conjecture implies that these Hecke algebras also control the representation theory of types of positive depth.

As mentioned before, for $\mathrm{GL}_{n}$ our theory specializes to Howe's theory, and can be expressed simply in terms of arithmetic of extensions of $F$. It is quite easy to do the same for all classical groups. We will treat this in another paper. A recent work of Julee Kim [Ki] also constructs supercuspidal representations of some classical groups, with more restrictions on the base field. It seems that all her representations can be obtained by our method, though we have not made a careful comparison.

We now give more technical comments about the contents.

The tameness condition is used in several places: (i) in Proposition 2.2 and Proposition 13.4, it is used to ensure the vanishing of a Galois cohomology, which is very important in this paper; (ii) it is used in $\sqrt{2}$ to ensure that our theory is non-empty; (iii) it simplifies the formulations in the case of classical groups. The usage in (iii) is not crucial. The usage in (ii) can be by-passed by other arguments in many wild cases. The usage in (i) presents more serious difficulties for generalizations. However, the real difficulty in the "wild" case is that considerably different constructions should be involved - as revealed in the $\mathrm{GL}_{n}$ case by the work of Bushnell, Corwin, and Kutzko ([BK1], [Co]$)$.

Though minimal $K$-types are not explicitly used in this paper, the overwhelming influence of the work of Moy and Prasad should be obvious. We also rely heavily on Bruhat-Tits theory throughout the paper, in particular, the theory of concave functions and their associated groups.

The basic ideas of the construction and the proof of supercuspidality are quite simple and are presented in $\$$ in an axiomatic way. This construction has also been considered by Adler independently and the main theorem in $\$$ was known to him in special cases (private communication). The basic strategy of $₫ 4$ has been used in many works in the literature. But the argument is incomplete in some papers. The crucial condition SC3 is an important key to complete the argument.

The central notion of the construction is that of generic characters and their intertwining properties. These properties should be applicable to study all irreducible admissible representations, though we concentrate on the supercuspidal ones in this paper. The first intertwining property (see SC1 in 84 and Theorem 9.4) is now quite standard, and a large part of the proof can be found in the works of Adler and Roche (and can be traced back to the works of Howe and Moy). What is new here is that we use the dual Lie algebra throughout. This is more natural and enables us to relax the restriction on the residual characteristic (see Proposition 7.3 and Lemma 8.1).

The second intertwining property (see SC2 in $\$ 4$ and Theorem 11.5) involves representations of Heisenberg groups and groups acting on Heisenberg groups. Here we need to lift a natural projective representation to an ordinary representation. One can find two approaches to this problem in the literature: either by showing that the obstruction for lifting vanishes, or by saying "from the theory of Weil representations, we can lift the representation". The second approach has the 
advantage of singling out a canonically defined lifting. However, most authors have not properly justified their usage of the second approach. See \$10 for more discussion of this problem and $\$ 11$ for our solution to this problem.

The third intertwining property (see SC3 in $\$ 4$ and Theorem 14.2) is a very rigid and pleasant property of generic characters, and it is most difficult to prove. Our approach, presented in $\$ 12$, 4 , and $\$ 14$, is partly inspired by Howe's paper [Ho. In proving this property, it is very important to have precise control of the lifting constructed in SC2.

We complete the proof of the main theorem in 15 , In $\$ 17$ we give a general Hecke algebra isomorphism associated to certain generic characters. This generalizes Howe-Moy's Hecke algebra isomorphism for "separated minimal $K$-types of tame $\mathrm{GL}_{n}$ " in [HM], and gives a criterion of non-supercuspidality. We conjecture that a similar isomorphism exists for every generic character. In the most general case, we can show that there is a support-preserving vector space isomorphism.

J. Adler's paper [Ad] has been very useful and inspiring for me during the development of this work. The notion of generic elements in this paper is a modification of his notion of good elements. I would like to thank him for making the paper available to me before its publication and for answering several of my questions. I also thank J. Adler, S. Debacker, and G. Prasad for their careful reading of a draft of this paper and numerous suggestions, and R. Howe, W.T. Gan, B.H. Gross, F. Murnaghan, P. Sally, and G. Savin for their interest and comments on this work.

\section{NOTATION AND CONVENTIONS}

Let $F$ be a non-archimedean local field of residual characteristic $p$, and let $\bar{F}$ be a fixed algebraic closure of $F$. We make no assumption on the value group of the valuation ord: $F \rightarrow \mathbb{R} \cup\{\infty\}$. However, subextensions of $\bar{F} / F$ are always endowed with the valuation extending the valuation ord on $F$.

All algebraic groups are assumed to be smooth. If $G$ is a linear algebraic group over $F$, we denote by $G^{\circ}$ the identity component of $G, z G$ the center of $G, \mathcal{D} G$ the derived group of $G$, Lie $G$ the Lie algebra of $G$, and $\operatorname{Lie}^{*} G$ the dual of Lie $G$. We often denote Lie $G$ and Lie* $G$ by the corresponding German letters, i.e. $\mathfrak{g}$ and $\mathfrak{g}^{*}$. They are vector spaces over $F$. We often write $\mathfrak{g}(E)$ for $\mathfrak{g} \otimes_{F} E=\operatorname{Lie}\left(G \otimes_{F} E\right)$.

Suppose that $G$ is reductive. We denote by $\mathcal{B}(G, F)$ the enlarged Bruhat-Tits building of $G$ over $F$. Recall that the enlarged building is the direct product of the reduced building by a real affine space. For any point $y$ in the enlarged building, we denote by $[y]$ the projection of $y$ on the reduced building, and by $G(F)_{y}$ (resp. $G(F)_{[y]}$ ) the subgroup of $G(F)$ fixing $y$ (resp. [y]). Following MoyPrasad [MP2], we let $G(F)_{y, 0}$ denote the (connected) parahoric subgroup associated with $y$.

For any finite extension $E$ of $F$, let $(E)_{r}=\{x \in E: \operatorname{ord}(x) \geq r\}$ for all $r \in \mathbb{R},(E)_{+}=\bigcup_{r>0}(E)_{r}$, and $\mathcal{O}_{E}=(E)_{0}$. Similarly, we put $\left(E^{\times}\right)_{0}=\mathcal{O}_{E}^{\times}$, $\left(E^{\times}\right)_{r}=1+(E)_{r}$ for $r>0$, and $\left(E^{\times}\right)_{+}=\bigcup_{r>0}\left(E^{*}\right)_{r}$.

We choose once and for all an additive character $\Psi$ of $F$ which is trivial on $(F)_{+}$ and non-trivial on $(F)_{0}$.

Suppose that $K$ is a subgroup of a group $G$, and $g \in G$. We denote $g K g^{-1}$ by ${ }^{g} K$, and ${ }^{g} K \cap K$ by ${ }^{g \cap} K$. If $\rho$ is a complex representation of $K,{ }^{g} \rho$ denotes the representation $x \mapsto \rho\left(g^{-1} x g\right)$ of ${ }^{g} K$, and $I_{g}(\rho)$ denotes the vector space $\operatorname{Hom}_{g \cap}{ }_{K}\left({ }^{g} \rho, \rho\right)$. If $I_{g}(\rho)$ is non-zero, we say that $g$ intertwines $\rho$. 
We use 1 to denote the trivial representation. Therefore, a representation is 1-isotypic if and only if the group acts trivially on the underlying vector space.

\section{Results from Bruhat-Tits theory: The split CAse}

Assume that $E$ is a finite extension of $F$ and $G$ is a split connected reductive group over $E$.

Let $T$ be a maximal $E$-split torus of $G$, and let $\Phi=\Phi(G, T, E)$ be the corresponding root system. For each $a \in \Phi$, let $G_{a}$ be the root subgroup corresponding to $a$. Choose a base $\Delta \subset \Phi$. For each subset $I \subset \Delta$, let $T_{I}$ be the identity component of $\bigcap_{a \in I} \operatorname{ker} a$, and let $G_{I}$ be the centralizer of $T_{I}$. Then $G_{I}$ is a Levi subgroup of a parabolic subgroup of $G$, and the center of $G_{I}$ has identity component $T_{I}$. Clearly, if $I \subset J$, then $T_{I} \supset T_{J}$ and $G_{I} \subset G_{J}$. The root system $\Phi_{I}=\Phi\left(G_{I}, T, E\right)$ is a subset of $\Phi$ and consists of precisely those roots in $\Phi$ which are integral linear combinations of elements of $I$.

Now suppose that we have a sequence of subsets of $\Delta$ :

$$
\emptyset \subseteq I_{0} \subsetneq I_{1} \subsetneq \cdots \subsetneq I_{d}=\Delta
$$

and suppose that $G^{i}=G_{I_{i}}$ are the corresponding groups, so

$$
T \subseteq G^{0} \subsetneq G^{1} \subsetneq \cdots \subsetneq G^{d}=G .
$$

In the sequel, most constructions depend only on $\vec{G}=\left(G^{0}, \ldots, G^{d}\right)$ and the choice of $(T, \Delta)$ is irrelevant (though occasionally a construction may appear to depend on the choice of $T$ ). We call the datum $\vec{G}$ a split Levi sequence in $G$.

Put $\Phi_{i}=\Phi_{I_{i}} \cup\{0\}=\Phi\left(G^{i}, T, E\right) \cup\{0\}$ so that $\{0\} \subseteq \Phi_{0} \subsetneq \Phi_{1} \subsetneq \cdots \subsetneq \Phi_{d}=$ $\Phi \cup\{0\}$. For each $a \in \Phi_{d}$, let $G_{a}$ be the root subgroup of $G$ corresponding to $a$ if $a \neq 0$, and $G_{a}=T$ if $a=0$. Let $\mathfrak{g}(E)$ be the Lie algebra of $G$, and let $\mathfrak{g}^{*}(E)$ be the dual of $\mathfrak{g}(E)$. For each $a \in \Phi_{d}$, let $\mathfrak{g}_{a}(E)$ (resp. $\mathfrak{g}_{a}^{*}(E)$ ) be the a-eigenspace of $\mathfrak{g}(E)$ (resp. $\mathfrak{g}^{*}(E)$ ) as a rational representation of $T$. Then $\mathfrak{g}_{a}(E)$ is the Lie algebra of $G_{a}$, and $\mathfrak{g}_{a}^{*}(E)$ is the dual of $\mathfrak{g}_{-a}(E)$.

We refer the reader to [BT1, 6.4.1] for the definition of $\tilde{\mathbb{R}}=\mathbb{R} \cup\{r+: r \in$ $\mathbb{R}\} \cup\{\infty\}$. We reproduce the following definition from [BT1, 6.4.3]: an $\tilde{\mathbb{R}}$-valued function $f$ on $\Phi \cup\{0\}$ is called concave if for any non-empty finite family $\left(a_{i}\right)$ of elements in $\Phi \cup\{0\}$ such that $\sum_{i} a_{i} \in \Phi \cup\{0\}$, we have

$$
f\left(\sum_{i} a_{i}\right) \leq \sum_{i} f\left(a_{i}\right)
$$

Lemma 1.1. Suppose that $f$ is an $\tilde{\mathbb{R}}$-valued function on $\Phi \cup\{0\}$ such that $0 \leq$ $\frac{1}{2} f(0) \leq f(a)$ for all $a \in \Phi$. Then $f$ is concave if and only if $f(a+b) \leq f(a)+f(b)$ for all $a, b, a+b \in \Phi$.

Proof. The "only if" part is obvious from the definition.

Let $\left(a_{i}\right)$ be a non-empty sequence of elements in $\Phi \cup\{0\}$ such that $a=\sum a_{i}$ is also in $\Phi \cup\{0\}$. We have to show that $f(a) \leq \sum f\left(a_{i}\right)$.

If $a \neq 0$, then $f(a) \leq \sum_{a_{i} \neq 0} f\left(a_{i}\right)$ by [BT1, 6.4.5], therefore, $f(a) \leq \sum f\left(a_{i}\right)$.

If $a=0$, the inequality $f(a) \leq \sum f\left(a_{i}\right)$ is obvious.

Consider a sequence $\vec{r}=\left(r_{0}, \ldots, r_{d}\right)$ of elements in $\tilde{\mathbb{R}}$ for which there exists $\nu \in \mathbb{Z}$ so that $0 \leq \nu \leq d$ and

$$
0 \leq r_{0}=\cdots=r_{\nu}, \frac{1}{2} r_{\nu} \leq r_{\nu+1} \leq \cdots \leq r_{d}
$$


We call such a sequence admissible. Define $f=f_{\vec{r}}: \Phi_{d} \rightarrow \tilde{\mathbb{R}}$ as follows: $f(a)=r_{0}$ if $a \in \Phi_{0}, f(a)=r_{k}$ if $a \in \Phi_{k} \backslash \Phi_{k-1}, 1 \leq k \leq d$.

Lemma 1.2. If $\vec{r}$ is admissible, the function $f_{\vec{r}}$ is concave.

Proof. We apply the preceding lemma. Suppose that $a, b, a+b \in \Phi_{d} \backslash\{0\}$. Let $j=\min \left\{j: a \in \Phi_{j}\right\}, k=\min \left\{k: b \in \Phi_{k}\right\}$. We may assume that $j \geq k$. Then $a+b \in \Phi_{j}$ and hence $f(a+b) \leq \max \left(r_{j}, r_{0}\right) \leq r_{j}+r_{k}=f(a)+f(b)$.

Let $y$ be in the apartment $A(G, T, E) \subset \mathcal{B}(G, E)$. Recall that $y$ determines filtration subgroups $\left\{G_{a}(E)_{y, r}\right\}_{r \in \tilde{\mathbb{R}}, r \geq 0}$ of $G_{a}(E)$, lattices $\left\{\mathfrak{g}_{a}(E)_{y, r}\right\}_{r \in \tilde{\mathbb{R}}}$ in $\mathfrak{g}_{a}(E)$, and lattices $\left\{\mathfrak{g}_{a}^{*}(E)_{y, r}\right\}_{r \in \tilde{\mathbb{R}}}$ in $\mathfrak{g}_{a}^{*}(E)_{y, r}$, for each $a \in \Phi_{d}$. If $a \neq 0$, the filtration of $G_{a}(E)$ can be extended to a filtration $\left\{G_{a}(E)_{y, r}\right\}_{r \in \tilde{\mathbb{R}}}$ indexed by the whole of $\tilde{\mathbb{R}}$.

For any $\tilde{\mathbb{R}}$-valued function $f$ on $\Phi_{d}$ such that $f(0) \geq 0$, let $G(E)_{y, f}$ be the subgroup generated by $G_{a}(E)_{y, f(a)}$ for all $a \in \Phi_{d}$, and let $\mathfrak{g}(E)_{y, f}\left(\operatorname{resp.} \mathfrak{g}^{*}(E)_{y, f}\right)$ be the lattice generated by $\mathfrak{g}_{a}(E)_{y, f(a)}\left(\right.$ resp. $\left.\mathfrak{g}_{a}^{*}(E)_{y, f(a)}\right)$ for all $a \in \Phi_{d}$. We will denote $G_{y, f_{\vec{r}}}$ by $\vec{G}(E)_{y, \vec{r}}$ and $\mathfrak{g}(E)_{y, f_{\vec{r}}}\left(\right.$ resp. $\left.\mathfrak{g}^{*}(E)_{y, f_{\vec{r}}}\right)$ by $\overrightarrow{\mathfrak{g}}(E)_{y, \vec{r}}\left(\operatorname{resp} \cdot \overrightarrow{\mathfrak{g}}^{*}(E)_{y, \vec{r}}\right)$. The notation is chosen to remind ourselves that $\vec{G}(E)_{y, \vec{r}}$ depends on $(\vec{G}, y, \vec{r})$. Notice that $\vec{G}(E)_{y, \vec{r}}$ is just an open subgroup of $G(E)$, not a sequence of groups.

Here is an alternative description of the lattices. Let $V$ be $\mathfrak{g}(E)$ or $\mathfrak{g}^{*}(E)$. Then $V$ is the direct sum of its $a$-eigenspaces $V_{a}, a \in \Phi_{d}$. Let $V^{0}=\sum_{a \in \Phi_{0}} V_{a}$, $V^{i}=\sum_{a \in \Phi_{i} \backslash \Phi_{i-1}} V_{a}$ for $i \geq 1$. Since each $V_{a}$ is equipped with a filtration $\left\{V_{a, y, r}\right\}$ (defined in MP1, MP2]), each $V^{i}$ is equipped with a filtration $\left\{V_{y, r}^{i}=\right.$ $\left.\sum_{a \in \Phi_{i} \backslash \Phi_{i-1}} V_{a, y, r}\right\}$. The lattice $V_{y, \vec{r}}$ is simply $V_{y, r_{0}}^{0} \oplus \cdots \oplus V_{y, r_{d}}^{d}$.

Let $\vec{r}, \vec{s}$ be two admissible sequences of elements in $\tilde{\mathbb{R}}$. We write $\vec{r}<\vec{s}$ (resp. $\vec{r} \leq$ $\vec{s})$ if $r_{i}<s_{i}$ (resp. $r_{i} \leq s_{i}$ ) for $0 \leq i \leq d$.

To simplify the notation, we put

$$
\vec{G}(E)_{y, \vec{r}: \vec{s}}=\vec{G}(E)_{y, \vec{r}} / \vec{G}(E)_{y, \vec{s}} \quad \text { and } \quad \overrightarrow{\mathfrak{g}}(E)_{y, \vec{r}: \vec{s}}=\overrightarrow{\mathfrak{g}}(E)_{y, \vec{r}} / \overrightarrow{\mathfrak{g}}(E)_{y, \vec{s}}
$$

whenever $\vec{r} \leq \vec{s}$.

Lemma 1.3. If $\vec{r}, \vec{s}$ are two admissible sequences such that

$$
0<r_{i} \leq s_{i} \leq \min \left(r_{i}, \ldots, r_{d}\right)+\min (\vec{r}) \quad \text { for } 0 \leq i \leq d,
$$

then $\vec{G}(E)_{y, \vec{r}: \vec{s}}$ is abelian and isomorphic to $\overrightarrow{\mathfrak{g}}(E)_{y, \vec{r}: \vec{s}}$.

Proof. First notice that $\vec{G}(E)_{y, \vec{s}}$ is a normal subgroup of $\vec{G}(E)_{y, \vec{r}}$ by the preceding lemma. To show that the quotient is abelian, it suffices to show that the commutator subgroup of $\vec{G}(E)_{y, \vec{r}}$ is contained in $\vec{G}(E)_{y, \vec{s}}$. This will be a consequence of BT1 6.4.44]. To verify the hypothesis there, assume that $\left(a_{i}\right),\left(b_{j}\right)$ are non-empty sequences of elements in $\Phi \cup\{0\}$ such that $c=\sum a_{i}+\sum b_{j} \in \Phi \cup\{0\}$. We need to show that $f_{\vec{s}}(c) \leq \sum f_{\vec{r}}\left(a_{i}\right)+\sum f_{\vec{r}}\left(b_{j}\right)$.

Let $k=\min \left\{k: c \in \Phi_{k}\right\}$, so $f_{\vec{s}}(c)=s_{k}$. If $k=0$, the desired inequality is clear. If $k>0$, then either there is some $i_{0}$ such that $a_{i_{0}} \notin \Phi_{k-1}$ or there is some $j_{0}$ such that $b_{j_{0}} \notin \Phi_{k-1}$. Therefore, either $f_{\vec{r}}\left(a_{i_{0}}\right)$ or $f_{\vec{r}}\left(b_{j_{0}}\right)$ is greater than or equal to $\min \left(r_{k}, \ldots, r_{d}\right) \geq f_{\vec{s}}(c)-\min (\vec{r})$. Since $f_{\vec{r}}\left(b_{j}\right)$ and $f_{\vec{r}}\left(a_{i}\right)$ are always greater than or equal to $\min (\vec{r})$, the desired inequality always holds.

The second statement follows from the first statement and [BT1, 6.4.48].

We now discuss the dependence on $T$. Since the definitions of $\vec{G}(E)_{y, \vec{r}}$, etc. apparently depend on $T$, we temporarily use a subscript $T$ on the left to indicate 
this dependence, for example, ${ }_{T} \vec{G}(E)_{y, \vec{r}}$. We have assumed that $y \in A(G, T, E) \subset$ $\mathcal{B}(G, E)$. Therefore, $y$ determines a valuation of the root datum of $(G, T, E)$ in the sense of [BT1]. This valuation restricted on the root datum of $\left(G^{i}, T, E\right)$, is a valuation there. Therefore, it determines a point $y_{i}={ }_{T} y_{i}$ in $A\left(G^{i}, T, E\right)$ modulo the action of $X_{*}\left(\mathcal{Z}\left(G^{i}\right), E\right) \otimes \mathbb{R}$. A choice of $y_{i}$ determines an embedding $j_{i}={ }_{T} j_{i}: \mathcal{B}\left(G^{i}, E\right) \rightarrow \mathcal{B}(G, E)$ which is $G^{i}(E)$-equivariant and maps $y_{i}$ to $y$.

Now suppose that $T^{\prime}$ is another maximal $E$-split torus of $G^{0}$ such that $y \in$ $A\left(G, T^{\prime}, E\right)$. Then ${ }_{T} y_{0} \in{ }_{T} j_{0}^{-1} A\left(G, T^{\prime}, E\right)=A\left(G^{0}, T^{\prime}, E\right)$. Therefore, there exists $g \in G^{0}(E)$ such that $\left.g \cdot{ }_{T} y_{0}\right)={ }_{T} y_{0}, g \cdot y=y$, and $T^{\prime}=g T g^{-1}$. Now it is easy to see that ${ }_{T}^{\prime} y_{0}=g \cdot\left({ }_{T} y_{0}\right)={ }_{T} y_{0}$ and ${ }_{T^{\prime}} \vec{G}(E)_{y, \vec{r}}=g\left({ }_{T} \vec{G}(E)_{y, \vec{r}}\right) g^{-1}={ }_{T} \vec{G}(E)_{y, \vec{r}}$. Therefore, $\vec{G}(E)_{y, \vec{r}}$ is independent of the choice of $T$.

Similarly we can show that $\overrightarrow{\mathfrak{g}}(E)_{y, r}, \overrightarrow{\mathfrak{g}}^{*}(E)_{y, r}$ are independent of $T$, and the isomorphism $\vec{G}(E)_{y, \vec{r}: \vec{s}} \rightarrow \overrightarrow{\mathfrak{g}}(E)_{y, \vec{r}: \vec{s}}$ in Lemma 1.3 is independent of $T$ (cf. [Ad] 1.6.6]).

If $\vec{G}=\left(G^{0}\right), G^{0}=G$, then $\vec{G}(E)_{y, \vec{r}}$ is simply the Moy-Prasad group $G(E)_{y, r_{0}}$ (see [MP1], [MP2]). When $\vec{r}$ is increasing, we can relate the more general $\vec{G}(E)_{y, \vec{r}}$ to the Moy-Prasad groups as follows.

Lemma 1.4. If $\vec{r}$ is an admissible increasing sequence, we have

$$
\vec{G}(E)_{y, \vec{r}}=G^{0}(E)_{y, r_{0}} G^{1}(E)_{y, r_{1}} \ldots G^{d}(E)_{y, r_{d}} .
$$

Here we abuse notation and identify $\mathcal{B}\left(G^{i}, E\right)$ with its image in $\mathcal{B}(G, E)$ under $j_{i}$, hence we identify $y_{i}$ with $y$.

Proof. Inductively, we show that $G^{0}(E)_{y, r_{0}} \ldots G^{i}(E)_{y, r_{i}}$ is a group. This is clear for $i=0$. For $i>0, G^{0}(E)_{y, r_{0}} \ldots G^{i-1}(E)_{y, r_{i-1}}$ is a group by our induction hypothesis. It is clearly a subgroup of $G^{i}(E)_{y, 0}$. Since $G^{i}(E)_{y, 0}$ normalizes $G^{0}(F)_{y, r_{0}} \ldots$ $G^{i-1}(E)_{y, r_{i-1}}$, we see that $G^{0}(E)_{y, r_{0}} \ldots G^{i-1}(E)_{y, r_{i-1}} G^{i}(E)_{y, r_{i}}$ is a group.

It is clear that $G^{0}(E)_{y, r_{0}} \ldots G^{d}(E)_{y, r_{d}}$ is generated by $G_{a}(E)_{y, f_{\vec{r}}(a)}$ for all $a \in$ $\Phi_{d}$.

It is possible to use more general $\vec{r}$ to construct concave functions. However, the admissible sequences are enough for our purpose. In fact, we only need two cases: either $\vec{r}$ is increasing, or $d=1$ and $\vec{r}=\left(r_{0}, r_{1}\right)$ satisfies $r_{0} / 2 \leq r_{1}$.

\section{Bruhat-Tits theory: The general Case}

Let $G$ be a connected reductive group over $F$, and let $S$ be a maximal $F$-split torus of $G$. Let $S_{1}$ be a torus of $G$, defined over $F$, such that $S_{1} \supset S$ and $S_{1} \otimes F_{1}$ is a maximal $F_{1}$-split torus of $G$, where $F_{1}$ is the maximal unramified extension of $G$. Such a torus exists by [BT2, 5.1.12]. Let $T_{1}$ be the centralizer of $S_{1}$. Then $T_{1}$ is a maximal torus of $G$ because $G$ is quasi-split over $F_{1}$.

Lemma 2.1. The following conditions are equivalent:

(i) There exists a tamely ramified extension $E / F$ such that $G \otimes E$ is split.

(ii) The splitting field $E_{1}$ of $T_{1}$ is tamely ramified over $F$.

Suppose that these two conditions hold. If $E / F$ is such that $G \otimes E$ is split, then $E_{1} F_{1} \subset E F_{1}$. 
Proof. It is clear that (ii) implies (i).

Assume (i). We may and do assume that $E \supset F_{1}$. Then there exists a maximal $E$-split torus $T$ of $G \otimes E$ containing $S_{1} \otimes E$. Such a torus is certainly contained in the centralizer $T_{1}$ of $S_{1}$, hence is equal to $T_{1} \otimes E$. Therefore, the splitting field $E_{1}$ of $T_{1}$, being a subfield of $E$, is tamely ramified. Thus we have $E_{1} F_{1} \subset E=E F_{1}$.

We shall say that $G$ is tamely ramified if the two equivalent conditions in the preceding lemma hold. The following observation will be useful later ( 13 ): if $G$ is tamely ramified, for any apartment $A(G, S, F)$ of $\mathcal{B}(G, F)$, there is a maximal torus $T$ which is split over a tamely ramified extension $E$, and $A(G, S, F) \subset A(G, T, E)$. In fact, we can simply take $T=T_{1}$ and apply a result of Rousseau ([Ti 2.6.1]).

Consider a sequence of reductive subgroups $T \subseteq G^{0} \subsetneq G^{1} \subsetneq \ldots \subsetneq G^{d}=G$ in $G$. We say that $\vec{G}=\left(G^{0}, \ldots, G^{d}\right)$ is a twisted Levi sequence in $G$ if there is a finite extension $E / F$ such that $G^{0} \otimes E$ is split, and $\vec{G} \otimes E=\left(G^{0} \otimes E, \ldots, G^{d} \otimes E\right)$ is a split Levi sequence in $G \otimes E$ (in the sense of 11 ). We call such an extension $E$ a splitting field of $\vec{G}$. If there is a splitting field $E$ of $\vec{G}$ which is Galois and tamely ramified, we say that the twisted Levi sequence $\vec{G}$ is tamely ramified.

Suppose that $\vec{G}$ is a twisted Levi sequence in $G$ and $T$ is a maximal torus of $G^{0}$ such that $T \otimes E$ is split. Then $A(G, T, E)$ and $\mathcal{B}(G, F)$ are both subsets of $\mathcal{B}(G, E)$. We put $A(G, T, F)=A(G, T, E) \cap \mathcal{B}(G, F)$. Notice that if $E^{\prime}$ is an extension of $E$, then $A(G, T, E)=A\left(G, T, E^{\prime}\right)$ in $\mathcal{B}\left(G, E^{\prime}\right)$. Therefore, the definition of $A(G, T, F)$ does not depend on the splitting field $E$. It is possible that $A(G, T, F)$ is empty. However, if $T$ (hence $\vec{G}$ ) has a tamely ramified Galois splitting field $E$, then $\operatorname{Gal}(E / F)$ acts on $A(G, T, E)$ by affine automorphisms. The center of mass of a $\operatorname{Gal}(E / F)$-orbit in $A(G, T, E)$ is certainly fixed by $\operatorname{Gal}(E / F)$, and is a point of $A(G, T, F)$ by a result of Rousseau ([Ti, 2.6.1]; a simple proof of this result of Rousseau has been provided by G. Prasad $[\mathrm{P}]$ ). This observation has been used by Adler in $\mathrm{Ad}]$.

Let $y \in A(G, T, F)$, and let $\vec{r}$ be an ( $\tilde{\mathbb{R}}$-valued) admissible sequence of length $d+1$. Then we can consider $\vec{G}(E)_{y, \vec{r}}$ as defined in the preceding section. We define $\vec{G}(F)_{y, \vec{r}}$ to be $\vec{G}(E)_{y, \vec{r}} \cap G(F)$. If $E^{\prime}$ is an extension of $E$, then we have $\vec{G}\left(E^{\prime}\right)_{y, \vec{r}} \cap G(E)=\vec{G}(E)_{y, \vec{r}}$. Therefore, the definition of $\vec{G}(F)_{y, \vec{r}}$ does not depend on the choice of $E$. If $E / F$ is Galois, then it is easy to see that $\vec{G}(E)_{y, \vec{r}}$ is Galois stable. Also, we can say that $\vec{G}(F)_{y, \vec{r}}$ is the group of Galois-fixed elements in $\vec{G}(E)_{y, \vec{r}}$.

The lattices $\overrightarrow{\mathfrak{g}}(F)_{y, \vec{r}}$ and $\overrightarrow{\mathfrak{g}}^{*}(F)_{y, \vec{r}}$ are defined in the same fashion. Again we define $\vec{G}(F)_{y, \vec{r}: \vec{s}}=\vec{G}(F)_{y, \vec{r}} / \vec{G}(F)_{y, \vec{s}}$ and define $\overrightarrow{\mathfrak{g}}(F)_{y, \vec{r}: \vec{s}}, \overrightarrow{\mathfrak{g}}^{*}(F)_{y, \vec{r}: \vec{s}}$ similarly. The quotient $\vec{G}(F)_{y, \vec{r}: \vec{s}}$ is abelian if $0<\vec{r} \leq \vec{s}$ and $s_{i} \leq \min \left(r_{i}, \ldots, r_{d}\right)+\min (\vec{r})$ for all $i$.

We would like to obtain results similar to those for split groups, at least when the twisted Levi sequence is tamely ramified.

In the statements of the following results, we are not assuming that $E / F$ is a splitting field of $\vec{G}$. However, for the rest of this section, we assume that $\vec{G}$ is tamely ramified.

Proposition 2.2. If $E / F$ is Galois and tamely ramified, and $\vec{s}>0$, then

$$
H^{1}\left(\operatorname{Gal}(E / F), \vec{G}(E)_{y, \vec{s}}\right) \text { and } H^{1}\left(\operatorname{Gal}(E / F), \overrightarrow{\mathfrak{g}}(E)_{y, \vec{s}}\right)
$$

are trivial. 
Corollary 2.3. If $E / F$ is Galois and tamely ramified, $0 \leq \vec{r} \leq \vec{s}$, and $\vec{s}>0$, then the natural homomorphisms

$$
\vec{G}(F)_{y, \vec{r}} \rightarrow\left(\vec{G}(E)_{y, \vec{r}: \vec{s})} \operatorname{Gal}(E / F)\right.
$$

and

$$
\overrightarrow{\mathfrak{g}}(F)_{y, \vec{r}} \rightarrow\left(\overrightarrow{\mathfrak{g}}(E)_{y, \vec{r}: \vec{s})} \operatorname{Gal}(E / F)\right.
$$

are surjective.

Corollary 2.4. If $0<\vec{r} \leq \vec{s}, s_{i} \leq \min \left(r_{i}, \ldots, r_{d}\right)+\min (\vec{r})$ for all $i$, and $E / F$ is a splitting field of $\vec{G}$ which is Galois and tamely ramified, then the isomorphism $\vec{G}(E)_{y, \vec{r}: \vec{s}} \rightarrow \overrightarrow{\mathfrak{g}}(E)_{y, \vec{r}: \vec{s}}$ induces an isomorphism

$$
\vec{G}(F)_{y, \vec{r}: \vec{s}} \rightarrow \overrightarrow{\mathfrak{g}}(F)_{y, \vec{r}: \vec{s}}
$$

Proof. The isomorphism $\vec{G}(E)_{y, \vec{r}: \vec{s}} \rightarrow \overrightarrow{\mathfrak{g}}(E)_{y, \vec{r}: \vec{s}}$ is $\operatorname{Gal}(E / F)$-equivariant. Therefore, it restricts to an isomorphism on the $\operatorname{Gal}(E / F)$-fixed subgroups. By the preceding corollary, the group of $\operatorname{Gal}(E / F)$-fixed points in $\vec{G}(E)_{y, \vec{r}: \vec{s}}\left(\right.$ resp. $\left.\overrightarrow{\mathfrak{g}}(E)_{y, \vec{r}: \vec{s}}\right)$ is precisely $\vec{G}(F)_{y, \vec{r}: \vec{s}}\left(\right.$ resp. $\overrightarrow{\mathfrak{g}}(F)_{y, \vec{r}: \vec{s}}$.

To prove the proposition, we need a few lemmas. The first two are general facts about the vanishing of $H^{1}$ (see [Se]).

Lemma 2.5. Suppose that $1 \rightarrow A \rightarrow B \rightarrow C \rightarrow 1$ is an exact sequence of $\operatorname{Gal}(E / F)$-groups. If $H^{1}(\mathrm{Gal}(E / F), A)$ and $H^{1}(\mathrm{Gal}(E / F), C)$ are both trivial, then $H^{1}(\mathrm{Gal}(E / F), B)$ is also trivial.

Lemma 2.6. Let $N / F$ be the maximal unramified subextension of $E / F$. Then we have an exact sequence $1 \rightarrow \operatorname{Gal}(E / N) \rightarrow \operatorname{Gal}(E / F) \rightarrow \operatorname{Gal}(N / F) \rightarrow 1$ of Galois groups and an exact sequence of cohomology sets

$$
1 \rightarrow H^{1}\left(\operatorname{Gal}(N / F), A^{\operatorname{Gal}(E / N)}\right) \rightarrow H^{1}(\operatorname{Gal}(E / F), A) \rightarrow H^{1}(\operatorname{Gal}(E / N), A),
$$

where $A$ is any topological group on which $\operatorname{Gal}(E / F)$ acts.

Therefore, in order to prove that $H^{1}\left(\operatorname{Gal}(E / F), A\right.$ ) (where $\left.A=\vec{G}(E)_{y, \vec{s}}\right)$ is trivial, it suffices to show that $H^{1}\left(\operatorname{Gal}(N / F), A^{\operatorname{Gal}(E / N)}\right)=H^{1}\left(\operatorname{Gal}(N / F), G(N)_{y, \vec{s}}\right)$ and $H^{1}(\operatorname{Gal}(E / N), A)$ are trivial. That is, it suffices to prove the statement when $E / F$ is unramified, and to prove it when $E / F$ is tamely and totally ramified.

Lemma 2.7. Suppose that $0<\vec{r} \leq \vec{s}, s_{i} \leq \min \left(r_{i}, \ldots, r_{d}\right)+\min (\vec{r})$ for all $i$. If $E / F$ is Galois and tamely ramified, then

$$
H^{1}\left(\operatorname{Gal}(E / F), \vec{G}(E)_{y, \vec{r}: \vec{s}}\right)=0 \text { and } H^{1}\left(\operatorname{Gal}(E / F), \overrightarrow{\mathfrak{g}}(E)_{y, \vec{r}: \vec{s}}\right)=0 .
$$

Lemma 2.8. Suppose that $\left\{A_{i}\right\}_{i \geq 0}$ is a decreasing sequence of $\operatorname{Gal}(E / F)$-groups such that each $A_{i}$ is normal in $A_{0}$ and $A_{0}$ is the inverse limit of $A_{0} / A_{i}$. If $H^{1}\left(\operatorname{Gal}(E / F), A_{0} / A_{i}\right)$ is trivial for all $i$, then $H^{1}\left(\operatorname{Gal}(E / F), A_{0}\right)$ is trivial.

Proof. Let $\Gamma=\operatorname{Gal}(E / F)$, and let $\left(a_{\gamma}\right)_{\gamma \in \Gamma}$ be a 1-cocycle with value in $A_{0}$. Then we can find $b_{0} \in A_{0}$ such that $\left(b_{0}^{-1} a_{\gamma} \gamma\left(b_{0}\right)\right)_{\gamma \in \Gamma}$ is a 1 -cocycle with value in $A_{1}$. Proceeding inductively, we can find $b_{i} \in A_{0}$ such that $b_{i} \equiv b_{i-1}$ modulo $A_{i}$ and $\left(b_{i}^{-1} a_{\gamma} \gamma\left(b_{i}\right)\right)_{\gamma \in \Gamma}$ is a 1-cocycle with value in $A_{i+1}$. Let $b=\lim b_{i}$; then $\left(b^{-1} a_{\gamma} \gamma(b)\right)_{\gamma \in \Gamma}$ is the trivial 1-cocycle. This proves the lemma. 
Before proving Lemma 2.7, we show that Proposition 2.2 follows from Lemmas 2.5 2.8 By Lemma 2.5] and Lemma 2.7 we easily see that

$$
H^{1}\left(\operatorname{Gal}(E / F), \vec{G}(E)_{y, \vec{r}: \vec{s}}\right)
$$

is trivial for all $0<\vec{r} \leq \vec{s}$. Now the proposition follows by applying Lemma 2.8 with $A_{i}=\vec{G}(E)_{y, \vec{s}+i}$, where $\vec{s}+i$ is the sequence $\left(r_{0}+i, r_{1}+i, \ldots, r_{d}+i\right)$.

We first prove Lemma 2.7 when $E / F$ is tamely and totally ramified. The cohomology groups in question are abelian groups killed by a power of $p$ and also by the order of $\operatorname{Gal}(E / F)$, which is prime to $p$. Therefore, they are zero.

Now assume that $E$ is actually a splitting field of $\vec{G}$, and $N / F$ is the maximal unramified subextension of $E / F$. By the special case of Lemma 2.7 (and also of the proposition and its corollaries) that we just proved, we now know that $\vec{G}(N)_{y, \vec{r}: \vec{s}}$ is isomorphic to $\overrightarrow{\mathfrak{g}}(N)_{y, \vec{r}: \vec{s}}$, the isomorphism is $\operatorname{Gal}(N / F)$-equivariant, and the two groups are precisely the subgroups of $\vec{G}(E)_{y, \vec{r}: \vec{s}}$ and $\overrightarrow{\mathfrak{g}}(E)_{y, \vec{r}: \vec{s}}$ fixed by $\operatorname{Gal}(E / N)$. By Lemma 2.6, to finish the proof of Lemma 2.7 (in the case that $E$ is a splitting field), it suffices to show that $H^{1}\left(\operatorname{Gal}(N / F), \overrightarrow{\mathfrak{g}}(N)_{y, \vec{r}: \vec{s}}\right)=0$. This is a consequence of the following lemma.

Lemma 2.9. If $N / F$ is unramified, $V$ is an $F$-vector space, and $L \supset M$ are $\operatorname{Gal}(N / F)$-stable lattices in $V \otimes N$, then

$$
H^{1}(\operatorname{Gal}(N / F), L / M)=0 .
$$

Proof. A $\operatorname{Gal}(N / F)$-stable lattice $L$ is necessarily of the form $L_{0} \otimes_{\mathcal{O}_{F}} \mathcal{O}_{N}$ : this is a simple consequence of the fact that $H^{1}\left(\operatorname{Gal}(N / F), \operatorname{GL}_{n}\left(\mathcal{O}_{N}\right)\right)=0$, which is a simple consequence of Hilbert's theorem 90 over the residue field.

Let $L_{0}, M_{0}$ be lattices in $V$ such that $L_{0} \otimes_{\mathcal{O}_{F}} \mathcal{O}_{N}=L, M_{0} \otimes_{\mathcal{O}_{F}} \mathcal{O}_{N}=M$. We can find a sequence of lattices $L_{0} \supset L_{1} \supset \ldots \supset L_{k}=M_{0}$ in $V$ such that $\pi_{F} L_{i} \subset L_{i+1}$. By Lemma 2.5 it suffices to prove that $H^{1}\left(\operatorname{Gal}(N / F),\left(L_{i} \otimes_{\mathcal{O}_{F}} \mathcal{O}_{N}\right) /\left(L_{i+1} \otimes_{\mathcal{O}_{F}}\right.\right.$ $\left.\left.\mathcal{O}_{N}\right)\right)=0$ for all $i$. This is again just the (additive) Hilbert's theorem 90 over the residue field. The lemma is proved.

Now Lemma 2.7 (and the proposition and its corollaries) is proved when $E / F$ is a splitting field of $\vec{G}$. For a general (Galois and tamely ramified) extension $E / F$, let $N / F$ again be the maximal unramified subextension of $E / F$. Lemma 2.6 tells us that in order to prove Lemma 2.7 we only have to prove (i) $H^{1}(\operatorname{Gal}(E / N), A)=0$, and (ii) $H^{1}\left(\operatorname{Gal}(N / F), A^{\operatorname{Gal}(E / N)}\right)=0$, where $A$ is either $\vec{G}(E)_{y, \vec{r}: \vec{s}}$ or $\overrightarrow{\mathfrak{g}}(E)_{y, \vec{r}: \vec{s}}$.

We have proved (i) in all cases. From this and the case we have already handled, we now know that $A^{\operatorname{Gal}(E / N)}$ is isomorphic to $\overrightarrow{\mathfrak{g}}(N)_{y, \vec{r}: \vec{s}}$. Therefore, (ii) follows from Lemma 2.9 again. This concludes the proof of Lemma 2.7 and of the proposition.

If $d=0, G^{0}=G$, and $r_{0}=r>0$, then $\vec{G}(F)_{y, \vec{r}}$ is simply Moy-Prasad's $G(F)_{y, r}$ by [Ad]. In general, $G(E)_{y, 0} \cap G(F), G(F)_{y, 0}, G(F)_{y}$, and $G(F)_{[y]}$ are all different. Again we have the following relation, which is not needed in the sequel.

Lemma 2.10. If $\vec{G}$ is tamely ramified and $\vec{r}$ is increasing with $r_{0}>0$, we have

$$
\vec{G}(F)_{y, \vec{r}}=G^{0}(F)_{y, r_{0}} G^{1}(F)_{y, r_{1}} \ldots G^{d}(F)_{y, r_{d}} .
$$

Remark 2.11. Again we need to explain the notation. We have assumed that $y \in$ $\mathcal{B}(G, F) \cap A(G, T, E)$, where $E / F$ is Galois and tamely ramified, and $T$ is a maximal torus of $G^{0}$ over $F$ such that $T \otimes E$ is split. Therefore, $y$ determines a point $y_{i}$ on 
$A\left(G^{i}, T, E\right)$ modulo the action of $X_{*}\left(\mathcal{Z}\left(G^{i}\right), E\right) \otimes \mathbb{R}$ as before. We may assume that $y_{i}$ is fixed by $\operatorname{Gal}(E / F)$ (first choose any $y_{i}$, then replace it by the center of mass of its $\operatorname{Gal}(E / F)$-orbit). Then $y_{i}$ is a point on $\mathcal{B}\left(G^{i}, F\right)$ by a result of Rousseau. The embedding $j_{i}: \mathcal{B}\left(G^{i}, E\right) \rightarrow \mathcal{B}(G, E), j_{i}\left(y_{i}\right)=y$, is Galois equivariant, hence induces an embedding $\mathcal{B}\left(G^{i}, F\right) \rightarrow \mathcal{B}(G, F)$ (again by the result of Rousseau). Again we identify $\mathcal{B}\left(G^{i}, F\right)$ with its image in $\mathcal{B}(G, F)$. Therefore, we identify $y_{i}$ with $y$.

We now prove the lemma. As in Lemma 1.4, we see that the product in the statement of the proposition is a group. First we notice that the proposition is known if $\vec{G}$ is split or if $d=0$. We now perform an induction on $d$.

Define $\vec{G}^{\prime}=\left(G^{1}, \ldots, G^{d}\right), \vec{s}=\left(r_{1}, \ldots, r_{d}\right)$. Now let $g \in \vec{G}(E)_{y, \vec{r}} \cap G(F)$. Then the image of $g$ in

$$
\vec{G}(E)_{y, \vec{r}} / \vec{G}^{\prime}(E)_{y, \vec{s}}=G^{0}(E)_{y, r_{0}} / G^{0}(E)_{y, r_{1}}
$$

is fixed by $\operatorname{Gal}(E / F)$. Therefore, we can find $g_{0} \in G^{0}(F)_{y, r_{0}}$ such that $g_{0}^{-1} g$ is in $\vec{G}^{\prime}(E)_{y, \vec{s}}$ by Corollary 2.3 (we may assume that $r_{1}>0$ without any loss of generality). Clearly, $g_{0}^{-1} g$ is also in $G(F)$, hence $g_{0}^{-1} g$ is in $\prod_{i=1}^{d} G^{i}(F)_{y, r_{i}}$ by the induction hypothesis. Finally, we get $g=g_{0}\left(g_{0}^{-1} g\right)$ is in $\prod_{i=0}^{d} G^{i}(F)_{y, r_{i}}$. This completes the proof.

\section{The DATUm}

We first recall the following fundamental result about depth zero supercuspidal representations, which is due to Moy and Prasad [MP2] and independently to Morris Mo1.

Theorem 3.1. Let $\pi$ be an irreducible supercuspidal representation of $G(F)$ such that the depth of $\pi$ is zero. Then there exists a maximal parahoric subgroup $G(F)_{y, 0}$ such that $\pi$ is compactly induced from a representation $\rho$ of the normalizer of $G(F)_{y, 0}$ such that $\rho \mid G(F)_{y, 0+}$ is 1-isotypic.

Remark 3.2. In the statement above, we used Moy-Prasad's notation that $G(F)_{y, 0}$ is the (connected) parahoric subgroup associated to $y$, as introduced before \$1. We caution the reader that there is a slight conflict of notation here: $G(F)_{y, 0}$ is in general different from $\vec{G}(F)_{y, \vec{r}}$ with $\vec{r}=(0, \ldots, 0)$, even if $d=0$. Since it is always the parahoric subgroup that is more interesting to us, we now make the convention that $G(F)_{y, 0}$ always means the parahoric subgroup.

Lemma 3.3. Assume the notation of the theorem.

(i) The normalizer of $G(F)_{y, 0}$ is equal to $G(F)_{[y]}$, the fixer of $[y]$ under the action of $G(F)$ on the reduced building of $G$.

(ii) If $\pi$ is also compactly induced from a representation $\rho^{\prime}$ of $G(F)_{\left[y^{\prime}\right]}$, with $\rho^{\prime} \mid G(F)_{y^{\prime}, 0+}$ being 1-isotypic, then $\left[y^{\prime}\right]=g$. [y] for some $g \in G(F)$.

Proof. (i) If $g G(F)_{y, 0} g^{-1}=G(F)_{g \cdot y, 0}$ is equal to $G(F)_{y, 0}$, then $g \cdot[y]=[y]$ by BT2, the proof of 5.2.8].

(ii) Then $\pi$ contains an unrefined minimal $K$-type of the form $\left(G(F)_{y, 0}, \chi\right)$ and another of the form $\left(G(F)_{y^{\prime}, 0}, \chi^{\prime}\right)$. By [MP1], this implies that there exists $g \in$ $G(F)$ such that $G(F)_{y^{\prime}, 0} \cap G(F)_{g . y, 0}$ maps surjectively onto both $G(F)_{y^{\prime}, 0: 0+}$ and $G(F)_{g . y, 0: 0+}$. But if $\left[y^{\prime}\right] \neq g$. $[y]$, then $G(F)_{y^{\prime}, 0} \cap G(F)_{g . y, 0}$ lies in a non-maximal parahoric subgroup, and cannot map surjectively onto $G(F)_{y^{\prime}, 0: 0+}$.

Therefore, we must have $\left[y^{\prime}\right]=g \cdot[y]$ and (ii) is proved. 
As mentioned in the introduction, the datum we shall use to construct a supercuspidal representation is a triple $\left(\vec{G}, \pi_{0}, \vec{\phi}\right)$. But it will be convenient to work with a 5 -tuple $(\vec{G}, y, \vec{r}, \rho, \vec{\phi})$ instead for a while. We now explain the meaning of this 5-tuple. Later (Remark [3.7] and Theorem 15.7] in \$15) we will show how the 5-tuple determines a triple, and that the construction only depends on the triple.

D1 $\vec{G}$ is a tamely ramified twisted Levi sequence $\vec{G}=\left(G^{0}, \ldots, G^{d}\right)$ in $G$; we assume that $Z\left(G^{0}\right) / Z(G)$ is anisotropic;

D2 $y$ is a point in $\mathcal{B}(G, F) \cap A(G, T, E)$, where $T$ is a maximal torus of $G^{0}$, and $E$ is a Galois tamely ramified splitting field of $T$ (hence of $\vec{G}$ ); this point is fixed throughout and is used in all the constructions, and will be suppressed from the notation;

D3 $\vec{r}=\left(r_{0}, \ldots, r_{d}\right)$ is a sequence of real numbers satisfying $0<r_{0}<r_{1}<\ldots<$ $r_{d-1} \leq r_{d}$ if $d>0,0 \leq r_{0}$ if $d=0$

D4 $\rho$ is an irreducible representation of $K^{0}=G^{0}(F)_{[y]}$ such that $\left.\rho\right|_{G^{0}(F)_{0+}}$ is 1-isotypic (recall that $G^{0}(F)_{0+}$ means $G^{0}(F)_{y, 0+}$ by the convention introduced in D2) and the compactly induced representation $\pi_{0}=\operatorname{ind}_{K^{0}}^{G^{0}(F)} \rho$ is irreducible supercuspidal;

D5 $\vec{\phi}=\left(\phi_{0}, \ldots, \phi_{d}\right)$ is a sequence of quasi-characters, where $\phi_{i}$ is a quasicharacter of $G^{i}(F)$; we assume that $\phi_{i}$ is trivial on $G^{i}(F)_{r_{i}+}$ but non-trivial on $G^{i}(F)_{r_{i}}$ for $0 \leq i \leq d-1$. If $r_{d-1}<r_{d}$, we assume that $\phi_{d}$ is trivial on $G^{d}(F)_{r_{d}}+$ but non-trivial on $G^{d}(F)_{r_{d}}$, otherwise we assume that $\phi_{d}=\mathbf{1}$.

Remark 3.4. Condition D1 implies that we essentially have no freedom in choosing embeddings $\mathcal{B}\left(G^{i}, F\right) \hookrightarrow \mathcal{B}(G, F)$. The embedding is unique up to translation by an element of $X_{*}\left(\mathcal{Z}\left(G^{0}\right), F\right) \otimes \mathbb{R}=X_{*}(\mathcal{Z}(G), F) \otimes \mathbb{R}$, and this choice has no effect on the formation of the groups $\vec{G}(F)_{y, \vec{s}}$, etc.

Remark 3.5. Here is another consequence of D1. Let $z \in K^{0}$. Then $z \cdot y=y+v$ for some $v \in X_{*}\left(Z\left(G^{0}\right), F\right) \otimes \mathbb{R}=X_{*}(Z(G), F) \otimes \mathbb{R}$. It is easy to see that $\vec{G}(F)_{y+v, \vec{s}}=$ $\vec{G}(F)_{y, \vec{s}}$ for any admissible $\vec{s}$ and any $v \in X_{*}(\mathcal{Z}(G), F) \otimes \mathbb{R}$. Therefore, $z$ normalizes any group of the form $\vec{G}(F)_{y, \vec{s}}$.

Remark 3.6. The representation to be constructed will be of depth $r_{d}$. In case $d=0$, our construction simply gives the depth zero representation $\pi_{0}=\operatorname{ind}_{K^{0}}^{G^{0}(F)} \rho$.

Remark 3.7. Condition $\mathbf{D} 4$ implies that $G^{0}(F)_{y, 0}$ is a maximal parahoric subgroup of $G^{0}(F)$ by [MP2, Prop. 6.8]. In other words, $[y]$ is a vertex on the reduced building of $G^{0}(F)$. Lemma 3.3 says that $y$ is actually determined by $\pi_{0}$ (up to conjugacy by $\left.G^{0}(F)\right)$.

It is also clear that $\vec{r}$ is determined by $\vec{\phi}$ by condition D5. Therefore, the 5 -tuple $(\vec{G}, y, \vec{r}, \rho, \vec{\phi})$ determines the triple $\left(\vec{G}, \pi_{0}, \vec{\phi}\right)$, and the triple determines (up to conjugacy) 4 objects out of 5 in the 5 -tuple, namely $(\vec{G}, y, \vec{r}, \vec{\phi})$. It will be shown in Theorem 15.7 of \$15 that our construction only depends on the triple.

Remark 3.8. Combining Remark 3.4 and Remark 3.7, we see that there are only finitely many possible $y$ (even $\left(y,\left.\rho\right|_{G^{0}(F)_{y, 0}}\right)$ ) up to conjugation and translation by $X_{*}\left(Z\left(G^{0}\right), F\right) \otimes \mathbb{R}$.

To ease the notation, we put $T^{i}=\left(\mathcal{Z}\left(G^{i}\right)\right)^{\circ}, s_{i}=r_{i} / 2$. 
Using the datum, we define $K_{+}^{0}=G^{0}(F)_{0+}$, and for $1 \leq i \leq d$,

$$
\left\{\begin{array}{l}
K^{i}=K^{0} G^{1}(F)_{s_{0}} \cdots G^{i}(F)_{s_{i-1}}=G^{0}(F)_{[y]} \vec{G}^{(i)}(F)_{\left(0, s_{0}, \ldots, s_{i-1}\right)}, \\
K_{+}^{i}=K_{+}^{0} G^{1}(F)_{s_{0}+} \cdots G^{i}(F)_{s_{i-1}+}=\vec{G}^{(i)}(F)_{\left(0+, s_{0}+, \ldots, s_{i-1}+\right)}
\end{array}\right.
$$

where $\vec{G}^{(i)}$ is the twisted Levi sequence $\left(G^{0}, \ldots, G^{i}\right)$. We also put

$$
\left\{\begin{array}{l}
J^{i}=\left(G^{i-1}, G^{i}\right)(F)_{\left(r_{i-1}, s_{i-1}\right)}, \\
J_{+}^{i}=\left(G^{i-1}, G^{i}\right)(F)_{\left(r_{i-1}, s_{i-1}+\right)} .
\end{array}\right.
$$

Then we have for $1 \leq i \leq d$,

$$
K^{i-1} J^{i}=K^{i}, \quad K_{+}^{i-1} J_{+}^{i}=K_{+}^{i} .
$$

\section{The construction}

Suppose that $0 \leq i \leq d-1$.

We consider $\mathfrak{g}=\operatorname{Lie} G$ as a rational representation of $T^{i}$, and decompose it into isotypic subspaces. Then $\mathfrak{g}^{i}=\operatorname{Lie} G^{i}$ is the maximal subspace on which $T^{i}$ acts trivially. Let $\mathfrak{n}^{i}$ be the sum of the remaining isotypic subspaces. Then $\mathfrak{g}(F)_{s}$ is a direct sum $\mathfrak{g}^{i}(F)_{s} \oplus \mathfrak{n}^{i}(F)_{s}$ for all $s$, where $\mathfrak{n}^{i}(F)_{s}$ is a lattice in $\mathfrak{n}^{i}(F)$ (this is because $y \in A(G, S, F)$ and $\left.S \supset T^{0} \supset T^{i}\right)$.

Recall that $\phi_{i}$ is a linear character of $G^{i}(F)$ of depth $r_{i}$. We have

$$
G^{i}(F)_{s_{i}+: r_{i}+} \simeq \mathfrak{g}^{i}(F)_{s_{i}+: r_{i}+} \subset \mathfrak{g}^{i}(F)_{s_{i}+: r_{i}+} \oplus \mathfrak{n}^{i}(F)_{s_{i}+: r_{i}+} \simeq G(F)_{s_{i}+: r_{i}+} .
$$

We extend $\left.\phi_{i}\right|_{\mathfrak{g}^{i}(F)_{s_{i}+}}$ to a linear character on $\mathfrak{g}^{i}(F)_{s_{i}+r_{i}+} \oplus \mathfrak{n}^{i}(F)_{s_{i}+: r_{i}+}$ by letting it be trivial on $\mathfrak{n}^{i}(F)_{s_{i}+r_{i}+}$. The corresponding character of $G(F)_{s_{i}+}$ is denoted by $\hat{\phi}_{i}$.

It is clear that $\left.\phi_{i}\right|_{G^{i}(F)_{s_{i}+}}=\left.\hat{\phi}_{i}\right|_{G^{i}(F)_{s_{i}+}}$. Therefore, there is a unique character on the group $K^{0} G^{i}(F)_{0} G(F)_{s_{i}+}$ extending both $\left.\phi_{i}\right|_{G^{i}(F)_{s_{i}}}$ and $\hat{\phi}_{i}$. We shall denote this character again by $\hat{\phi}_{i}$. By convention we set $\hat{\phi}_{d}=\phi_{d}$. Notice that by construction, $\hat{\phi}_{i}$ is trivial on $\left(G^{i}, G\right)(F)_{\left(r_{i}+, s_{i}+\right)}$.

We now assume that for $0 \leq i \leq d-1, \phi_{i}$ satisfies the following condition:

$\mathbf{S C 1}_{i}$ If $g \in G^{i+1}(F)$ intertwines $\left.\hat{\phi}_{i}\right|_{J_{+}^{i+1}}$, then $g \in J^{i+1} G^{i}(F) J^{i+1}$.

Proposition 4.1. If $g \in G^{i}(F)$ intertwines $\theta_{i}=\left.\prod_{j=0}^{i} \hat{\phi}_{j}\right|_{K_{+}^{i}}$, then $g \in K^{i} G^{0}(F) K^{i}$.

Proof. We will prove the proposition by induction. The statement is trivial when $i=0$. Now suppose that $i \geq 1$.

Since $\left.\hat{\phi}_{i}\right|_{K_{+}^{i}}$ is the restriction of $\phi_{i}$, which is defined on the whole of $G^{i}(F), g$ also intertwines the character $\theta^{\prime}=\theta_{i} \hat{\phi}_{i}^{-1}=\left.\prod_{j=0}^{i-1} \hat{\phi}_{j}\right|_{K_{+}^{i}}$. Therefore, it also intertwines $\left.\theta^{\prime}\right|_{J_{+}^{i}}$. But $\hat{\phi}_{j}$ is trivial on $J_{+}^{i}$ for $j<i-1$. So $\left.\theta^{\prime}\right|_{J_{+}^{i}}=\left.\hat{\phi}_{i-1}\right|_{J_{+}^{i}}$. By $\mathbf{S C} \mathbf{1}_{i-1}$, there are $j_{1}, j_{2}, g^{\prime}$ such that $g=j_{1} g^{\prime} j_{2}$, and $j_{1}, j_{2} \in J^{i}, g^{\prime} \in G^{i-1}(F)$.

By Lemma 4.3 below, $g^{\prime}$ intertwines $\theta^{\prime}$ as well. Therefore, $g^{\prime}$ intertwines $\left.\theta^{\prime}\right|_{K_{+}^{i-1}}=$ $\theta_{i-1}$. By the induction hypothesis, $g^{\prime} \in K^{i-1} G^{0}(F) K^{i-1}$, and therefore, $g \in$ $K^{i} G^{0}(F) K^{i}$.

Lemma 4.2. With the notation of the above proof, we have

$$
\left[J^{i}, K_{+}^{i}\right] \subset\left(G^{i-1}, G^{i}\right)(F)_{\left(r_{i-1}+, s_{i-1}+\right)} \subset \operatorname{ker}\left(\theta^{\prime}\right) .
$$


Proof. It suffices to prove the first inclusion when $\vec{G}$ is split over $F$. In this case, the result is immediate from [BT1, 6.4.44]. In fact, we have $\left[J^{i}, G^{i}(F)_{0+}\right] \subset$ $\left(G^{i-1}, G^{i}\right)(F)_{\left(r_{i-1}+s_{i-1}+\right)}$. The second inclusion is obvious: $\hat{\phi}_{j}$ is trivial on $\left(G^{i-1}, G^{i}\right)(F)_{\left(r_{i-1}+s_{i-1}+\right)}$ for $0 \leq j \leq i-1$.

Lemma 4.3. Assume the notation is as in the proof of the proposition. If $g$ intertwines $\theta^{\prime}, j \in J^{i}$, then $g j$ and $j g$ also intertwine $\theta^{\prime}$.

Proof. Let $K=K_{+}^{i}$. Recall that $g$ intertwines $\theta^{\prime}$ if and only if $\theta^{\prime}\left(g^{-1} x g\right)=\theta^{\prime}(x)$ for all $x \in g K^{-1} \cap K$. It is clear from Lemma 4.2 that every $j \in J^{i}$ intertwines $\theta^{\prime}$. Lemma 4.2 also implies that $J^{i}$ normalizes $K$.

Suppose that $g$ intertwines $\theta^{\prime}$, and $j \in J^{i}$. Then for all $x \in(g j) K(g j)^{-1} \cap K=$ $g K g^{-1} \cap K$, we have $\theta^{\prime}\left((g j)^{-1} x(g j)\right)=\theta^{\prime}\left(j^{-1}\left(g^{-1} x g\right) j\right)=\theta^{\prime}\left(g^{-1} x g\right)=\theta^{\prime}(x)$. So $g j$ intertwines $\theta^{\prime}$. Similarly, for all $x \in(j g) K(j g)^{-1} \cap K=j\left(g K g^{-1} \cap K\right) j^{-1}$, we have $\theta^{\prime}\left((j g)^{-1} x(j g)\right)=\theta^{\prime}\left(g^{-1}\left(j^{-1} x j\right) g\right)=\theta^{\prime}\left(j^{-1} x j\right)=\theta^{\prime}(x)$. So $j g$ intertwines $\theta^{\prime}$. The lemma is proved.

Next, we assume that for $0 \leq i \leq d-1, \phi_{i}$ satisfies the following condition:

SC2 ${ }_{i}$ There is an irreducible representation $\tilde{\phi}_{i}$ of $K^{i} \ltimes J^{i+1}$ such that (i) the restriction of $\tilde{\phi}_{i}$ to $J_{+}^{i+1}=1 \ltimes J_{+}^{i+1}$ is $\left(\left.\hat{\phi}_{i}\right|_{J_{+}^{i+1}}\right)$-isotypic; and (ii) the restriction of $\tilde{\phi}_{i}$ to $K_{+}^{i} \ltimes 1$ is $\mathbf{1}$-isotypic.

We now construct a representation $\rho_{i}$ of $K^{i}$. The construction below depends on the choices of $\left(\tilde{\phi}_{i}\right)$ satisfying SC2. But later we will make canonical choices using the theory of Weil representations.

We will first construct a representation $\rho_{i}^{\prime}$ of $K^{i}$ such that $\left.\rho_{i}^{\prime}\right|_{G^{i}(F)_{r_{i}}}$ is 1-isotypic. We will then put $\rho_{i}=\rho_{i}^{\prime} \otimes\left(\left.\phi_{i}\right|_{K^{i}}\right)$. We proceed inductively. First, we put $\rho_{0}^{\prime}=\rho$ and $\rho_{0}=\rho_{0}^{\prime} \otimes\left(\left.\phi_{0}\right|_{K^{0}}\right)$.

Suppose that $\rho_{i-1}^{\prime}$ and $\rho_{i-1}$ are already constructed. We inflate $\left.\phi_{i-1}\right|_{K^{i-1}}$ to a representation $\inf \left(\phi_{i-1}\right)$ of $K^{i-1} \ltimes J^{i}$. We now claim that $\inf \left(\phi_{i-1}\right) \otimes \tilde{\phi}_{i-1}$ factors through the natural map $K^{i-1} \ltimes J^{i} \rightarrow K^{i-1} J^{i}=K^{i}$.

The kernel of this natural map consists of elements of the form $x \ltimes x^{-1}, x \in$ $K^{i-1} \cap J^{i}=G^{i-1}(F)_{r_{i-1}} \subset K_{+}^{i-1}$. For such an element, $\inf \left(\phi_{i-1}\right)\left(x \ltimes x^{-1}\right)$ is scalar multiplication by $\hat{\phi}_{i-1}(x)$, and $\tilde{\phi}_{i-1}\left(x \ltimes x^{-1}\right)$ is scalar multiplication by $\hat{\phi}_{i-1}\left(x^{-1}\right)$ by SC2. This proves the claim.

Let $\phi_{i-1}^{\prime}$ be the representation of $K^{i}$ whose inflation to $K^{i-1} \ltimes J^{i}$ is $\inf \left(\phi_{i-1}\right) \otimes$ $\tilde{\phi}_{i-1}$. Inflate $\rho_{i-1}^{\prime}$ to a representation $\inf \left(\rho_{i-1}^{\prime}\right)$ of $K^{i}=K^{i-1} J^{i}$ via the map $K^{i} \rightarrow K^{i-1} J^{i} / J^{i}=K^{i-1} /\left(K^{i-1} \cap J^{i}\right)$ (recall that $\rho_{i-1}^{\prime}$ restricted on $K^{i-1} \cap J^{i}$ is 1-isotypic). Now we define $\rho_{i}^{\prime}=\inf \left(\rho_{i-1}^{\prime}\right) \otimes \phi_{i-1}^{\prime}$, and $\rho_{i}=\rho_{i}^{\prime} \otimes\left(\left.\phi_{i}\right|_{K^{i}}\right)$. This completes the construction.

Proposition 4.4. $\left.\rho_{i}\right|_{K_{+}^{i}}$ is $\theta_{i}$-isotypic, where $\theta_{i}=\left.\prod_{j=0}^{i} \hat{\phi}_{j}\right|_{K_{+}^{i}}$.

Proof. We show this by induction. This is clear when $i=0$.

If $i \geq 1, x \in K_{+}^{i-1}, z \in J_{+}^{i}$, then $\inf \left(\rho_{i-1}^{\prime}\right)(x z)$ is scalar multiplication by $\theta_{i-1}(x) \hat{\phi}_{i-1}(x)^{-1}$ by the induction hypothesis, and $\phi_{i-1}^{\prime}(x z)$ is scalar multiplication by $\hat{\phi}_{i-1}(x) \hat{\phi}_{i-1}(z)$. This shows that $\rho_{i}^{\prime}(x z)$ is scalar multiplication by $\theta_{i-1}(x) \hat{\phi}_{i-1}(z)$. 
But $\hat{\phi}_{j}(z)=1$ for $j<i-1$. So $\rho_{i}^{\prime}(x z)$ is scalar multiplication by $\prod_{j=0}^{i-1} \hat{\phi}_{j}(x z)$, and $\rho_{i}(x z)$ is scalar multiplication by $\prod_{j=0}^{i} \hat{\phi}_{i}(x z)=\theta_{i}(x z)$. The proposition is proved.

Corollary 4.5. If $g \in G^{i}(F)$ intertwines $\rho_{i}$, then $g \in K^{i} G^{0}(F) K^{i}$.

Finally, we impose the following condition for $0 \leq i \leq d-1$ :

$\mathbf{S C 3}_{i}$ For all $g \in G^{0}(F)$, we have $\operatorname{dim} I_{g}\left(\left.\phi_{i}^{\prime}\right|_{J^{i+1}}\right)=\operatorname{dim} I_{g}\left(\left.\phi_{i}^{\prime}\right|_{K^{i+1}}\right)=1$.

Notice that we have a trivial inclusion: $I_{g}\left(\left.\phi_{i}^{\prime}\right|_{K^{i+1}}\right) \subset I_{g}\left(\left.\phi_{i}^{\prime}\right|_{J^{i+1}}\right)$. Therefore, condition $\mathbf{S C 3}_{i}$ asserts that these two spaces are equal and are both 1-dimensional.

Proposition 4.6. Suppose that $\mathbf{S C 1}_{i}, \mathbf{S C 2}_{i}, \mathbf{S C 3}_{i}$ are satisfied for $0 \leq i \leq d-$ 1. Then the compactly induced representation $\operatorname{ind}_{K^{i}}^{G^{i}(F)} \rho_{i}$ of $G^{i}(F)$ is irreducible supercuspidal of depth $r_{i}$ for $0 \leq i \leq d$.

Proof. The depth statement is easy.

By well-known results, it is necessary and sufficient to prove the following statement: if $g \in G^{i}(F)$ intertwines $\rho_{i}$, then $g \in K^{i}$. By Corollary 4.5 we may assume that $g \in G^{0}(F)$. We notice that $g$ intertwines $\rho_{i}$ if and only if $g$ intertwines $\rho_{i}^{\prime}$.

We proceed by induction using an argument in [BK1, 5.3.2]. Suppose that $f$ is a non-zero element in $I_{g}\left(\rho_{i}^{\prime}\right)$. Let $V$ and $W$ be the space of $\inf \left(\rho_{i-1}^{\prime}\right)$ and $\phi_{i-1}^{\prime}$ respectively. Then $V \otimes W$ is the space of $\rho_{i}^{\prime}$ and $f$ is an endomorphism of $V \otimes W$. We may write $f=\sum_{j} f_{j}^{\prime} \otimes f_{j}^{\prime \prime}$ such that $f_{j}^{\prime} \in \operatorname{End}(V)$ and $f_{j}^{\prime \prime} \in \operatorname{End}(W)$. Furthermore, we may assume that $\left\{f_{j}^{\prime}: j\right\}$ is a linearly independent set.

Let $x \in{ }^{g \cap} J^{i}$; then $\inf \left(\rho_{i-1}^{\prime}\right)(x)$ and ${ }^{g} \inf \left(\rho_{i-1}^{\prime}\right)(x)$ are both trivial. Since $f \in$ $I_{g}\left(\inf \left(\rho_{i-1}^{\prime}\right) \otimes \phi_{i-1}^{\prime}\right)$, we have

$\sum f_{j}^{\prime} \circ{ }^{g} \inf \left(\rho_{i-1}^{\prime}\right)(x) \otimes f_{j}^{\prime \prime} \circ{ }^{g} \phi_{i-1}^{\prime}(x)=\sum \inf \left(\rho_{i-1}^{\prime}\right)(x) \circ f_{j}^{\prime} \otimes \phi_{i-1}^{\prime}(x) \circ f_{j}^{\prime \prime}$.

Therefore, for $x \in g \cap J^{i}$ we have

$$
\sum f_{j}^{\prime} \otimes f_{j}^{\prime \prime} \circ{ }^{g} \phi_{i-1}^{\prime}(x)=\sum f_{j}^{\prime} \otimes \phi_{i-1}^{\prime}(x) \circ f_{j}^{\prime \prime} .
$$

The linear independence of the set $\left\{f_{j}^{\prime}: j\right\}$ implies that $f_{j}^{\prime \prime} \in I_{g}\left(\left.\phi_{i-1}^{\prime}\right|_{J^{i}}\right)$. Since $I_{g}\left(\left.\phi_{i-1}^{\prime}\right|_{J^{i}}\right)$ is 1-dimensional by SC3, we may assume that there is only one $j$. In other words, $f=f^{\prime} \otimes f^{\prime \prime}$, where $f^{\prime \prime}$ is a fixed basis of $I_{g}\left(\left.\phi_{i-1}^{\prime}\right|_{J^{i}}\right)=I_{g}\left(\left.\phi_{i-1}^{\prime}\right|_{K^{i}}\right)$.

It then follows easily that $f^{\prime}$ is a non-zero element in

$$
I_{g}\left(\inf \left(\rho_{i-1}^{\prime}\right)\right) \subset I_{g}\left(\rho_{i-1}^{\prime}\right)
$$

(observe that $\left.\left.\inf \left(\rho_{i-1}^{\prime}\right)\right|_{K^{i-1}}=\rho_{i-1}^{\prime}\right)$. By the induction hypothesis, this implies that $g \in G^{0}(F) \cap K^{i-1}=K^{0}$. This completes the proof.

\section{Duality And Intertwining}

Recall that if $L$ is a lattice in an $F$-vector space $V$, the dual lattice $L^{*}$ is defined to be $\left\{x \in V^{*}: x(L) \subset(F)_{0}\right\}$. We define $L^{\bullet}=L^{*} \otimes(F)_{+}$. If $L \subset M$ are lattices in $V$, then the Pontrjagin dual of $M / L$ can be identified with $L^{\bullet} / M^{\bullet}$ (via $\Psi$ ). Explicitly, every element $a \in L^{\bullet}$ defines a character $\chi=\chi_{a}$ on $M$ by $\chi_{a}(m)=\Psi(a(m))$. Clearly, $\chi_{a}$ factors through $M \mapsto M / L$ and $\chi_{a}$ depends on $a \bmod M^{\bullet}$ only. We say that a realizes the character $\chi$.

If $\vec{r}=\left(r_{0}, \ldots, r_{d}\right)$ is an $\mathbb{R}$-valued sequence, we define $\vec{r}+$ to be the sequence $\left(r_{0}+, \ldots, r_{d}+\right)$. Then $\mathfrak{g}^{*}(F)_{y, \vec{r}}$ is equal to $\left(\mathfrak{g}(F)_{y,(-\vec{r})+}\right)^{*} \otimes(F)_{+}$and $\mathfrak{g}^{*}(F)_{y, \vec{r}+}$ is equal to $\left(\mathfrak{g}(F)_{y,-\vec{r}}\right)^{*} \otimes(F)_{+}$. 
In fact, let $V$ be either $\mathfrak{g}(F)$ or $\mathfrak{g}^{*}(F)$, as a rational representation of a maximal torus $S$ of $G^{0}$. Let $T^{i} \subset S$ be the identity component of the center of $G^{i}$. Let $V_{i}$ be the maximal subspace of $V$ on which $T^{i}$ acts trivially, $V^{0}=V_{0}$, and let $V^{i}$ be the subspace of $V_{i}$ which is the direct sum of the non-trivial isotypic components under the action of $T^{i-1}$ if $i \geq 1$. Then $V=V^{0} \oplus \cdots \oplus V^{d}$. Each $V^{i}$ is equipped with an admissible filtration $\left\{V_{y, r}^{i}\right\}$, and $V_{y, \vec{r}}=V_{y, r_{0}}^{0} \oplus \cdots \oplus V_{y, r_{d}}^{d}$. For any $i$ and any real number $r,\left(V^{*}\right)_{y, r}^{i}$ is equal to $\left(V_{y,(-r)+}^{i}\right)^{*} \otimes(F)_{+}$, and $\left(V^{*}\right)_{y, r+}^{i}$ is equal to $\left(V_{y,-r}^{i}\right)^{*} \otimes(F)_{+}$.

Let $r>0$ and let $S$ be any group lying between $G(F)_{y,(r / 2)+}$ and $G(F)_{y, r}$. Let $\chi=\chi_{a}$ be a character of $S / G(F)_{y, r+} \simeq \mathfrak{s} / \mathfrak{g}(F)_{y, r+}$, where $\mathfrak{s}$ is a lattice between $\mathfrak{g}(F)_{y,(r / 2)+}$ and $\mathfrak{g}(F)_{y, r}$, and $a \in \mathfrak{g}^{*}(F)_{y,-r}=\left(\mathfrak{g}(F)_{y, r+}\right)^{\bullet}$. The following result is due to Adler $[\mathrm{Ad}]$.

Lemma 5.1. Let $g \in G$. Then $g$ intertwines $\chi_{a}$ if and only if $\operatorname{Ad}(g) \cdot\left(a+\mathfrak{s}^{\bullet}\right) \cap$ $\left(a+\mathfrak{s}^{\bullet}\right) \neq \emptyset$.

\section{Computing with a Chevalley basis}

In this section only, $E$ is an arbitrary field and $G$ is a split connected reductive group over $E$. Let $T$ be a maximal split torus of $G$ over $E$.

Then there exists a Chevalley system for $(G, T)$, which is a collection of isomorphisms $\left\{x_{a}: \mathbb{G}_{\mathrm{a}} \rightarrow G_{a}\right\}_{a \in \Phi(G, T, E)}$ with certain properties; see [BT2 or [Ad]. We now recall the basic facts and set up some notation.

For $a \in \Phi=\Phi(G, T, E)$, let $X_{a}=d x_{a}(1)$ and $H_{a}=\left[X_{a}, X_{-a}\right]=d a^{\vee}(1)\left(a^{\vee}\right.$ being the coroot of $a$ ). Then $X_{a}$ is a generator of the 1-dimensional space $\mathfrak{g}_{a}$. Let $X_{a}^{*}$ be the element of $\mathfrak{g}^{*}$ such that $X_{a}^{*}(\operatorname{Lie} T)=0, X_{a}^{*}\left(X_{b}\right)=\delta_{b,-a}$. Then $X_{a}^{*}$ is a generator of $\mathfrak{g}_{a}^{*}=\left(\mathfrak{g}^{*}\right)_{a}$, the $a$-eigenspace of $T$ (by the coadjoint action). Let $H_{a}^{*}$ be the element of $\mathfrak{g}^{*}$ such that $H_{a}^{*}\left(\mathfrak{g}_{b}\right)=0$ for all $b \in \Phi$, and $H_{a}^{*}(H)=d a(H)$ for $H \in \operatorname{Lie} T$.

As before, we shall write $G_{0}=T$ and $\mathfrak{g}_{0}=\operatorname{Lie} T$, the zero-eigenspace of $T$ acting on $\mathfrak{g}$, and we write $\left(\mathfrak{g}^{*}\right)_{0}$ for the zero-eigenspace of $T$ acting on $\mathfrak{g}^{*}$.

We then have the following formulas:

Lemma 6.1. Let $t \in T(E), a \in \Phi, u, v \in E^{*}$. Then

$$
\begin{array}{rlrl}
\operatorname{Int}(t) \cdot h & =h & & \text { for all } h \in G_{0}(E), \\
\operatorname{Int}(t) \cdot x_{b}(v) & =x_{b}(b(t) v) & & \text { for all } b \in \Phi, \\
\operatorname{Int}\left(x_{a}(u)\right) \cdot h & =x_{a}(u-a(h) \cdot u) h & & \text { for all } h \in G_{0}(E), \\
\operatorname{Int}\left(x_{a}(u)\right) \cdot x_{a}(v) & =x_{a}(v), & & \\
\operatorname{Int}\left(x_{a}(u)\right) \cdot x_{-a}(v) & =x_{-a}\left(v(1+u v)^{-1}\right) a^{\vee}(1+u v) & x_{a}\left(-u^{2} v(1+u v)^{-1}\right), \\
\operatorname{Int}\left(x_{a}(u)\right) \cdot x_{b}(v) & =\left(\prod_{i, j>0} x_{i a+j b}\left(C_{a, b ; i, j} u^{i} v^{j}\right)\right) x_{b}(v) & & \text { for all } b \in \Phi, b \neq \pm a .
\end{array}
$$

Lemma 6.2. Let $t \in T(E), a \in \Phi, u \in E^{*}$. Then

$$
\begin{aligned}
\operatorname{Ad}(t) \cdot H & =H & & \text { for all } H \in \mathfrak{g}_{0}, \\
\operatorname{Ad}(t) \cdot X_{b} & =b(t) X_{b} & & \text { for all } b \in \Phi, \\
\operatorname{Ad}\left(x_{a}(u)\right) \cdot H & =H-u H_{a}^{*}(H) X_{a} & & \text { for all } H \in \mathfrak{g}_{0}, \\
\operatorname{Ad}\left(x_{a}(u)\right) \cdot X_{a} & =X_{a}, & &
\end{aligned}
$$




$$
\begin{aligned}
\operatorname{Ad}\left(x_{a}(u)\right) \cdot X_{-a} & =X_{-a}+u H_{a}-u^{2} X_{a}, \\
\operatorname{Ad}\left(x_{a}(u)\right) \cdot X_{b} & =\sum_{i \geq 0} M_{a, b, i} u^{i} X_{i a+b} \quad \text { for all } b \in \Phi, b \neq \pm a .
\end{aligned}
$$

These results are well known. Here $C_{a, b ; i, j}$ and $M_{a, b, i}$ are integers, and $M_{a, b, 0}=$ 1. The product in Lemma 6.1 is taken in any fixed order. See [BT2, 3.2.3] for details. We put $M_{a, b, i}^{*}=M_{a,-(i a+b), i}$ in the next lemma. So $M_{a, b, 0}^{*}=1$ also.

Lemma 6.3. Let $t \in T(E), a \in \Phi, u \in E^{*}$. Then

$$
\begin{aligned}
\operatorname{Ad}(t) \cdot H^{*} & =H^{*} & & \text { for all } H^{*} \in\left(\mathfrak{g}^{*}\right)_{0}, \\
\operatorname{Ad}(t) \cdot X_{b}^{*} & =b(t) X_{b}^{*} & & \text { for all } b \in \Phi, \\
\operatorname{Ad}\left(x_{a}(u)\right) \cdot H^{*} & =H^{*}-u H^{*}\left(H_{a}\right) X_{a}^{*} & & \text { for all } H^{*} \in\left(\mathfrak{g}^{*}\right)_{0}, \\
\operatorname{Ad}\left(x_{a}(u)\right) \cdot X_{a}^{*} & =X_{a}^{*}, & & \\
\operatorname{Ad}\left(x_{a}(u)\right) \cdot X_{-a}^{*} & =X_{-a}^{*}+u H_{a}^{*}-u^{2} X_{a}^{*}, & & \\
\operatorname{Ad}\left(x_{a}(u)\right) \cdot X_{b}^{*} & =\sum_{i \geq 0} M_{a, b, i}^{*} u^{i} X_{i a+b}^{*} & & \text { for all } b \in \Phi, b \neq \pm a .
\end{aligned}
$$

Proof. This is immediate from the preceding lemma.

\section{Centralizers of Semisimple Elements}

In this section only, $E$ is an arbitrary algebraically closed field, $G$ is a connected reductive group over $E$, and $T$ is a maximal torus of $G$.

We refer to $[\mathrm{Sp}$ for the notion of root datum. (N.B. This notion is completely different from that in BT1.) We always assume that the root system of a root datum is reduced. Following [Sp], we write $\psi(G, T)=\left(X^{*}(T), \Phi(G, T), X_{*}(T), \Phi(G, T)^{\vee}\right)$ for the root datum of $(G, T)$ if $T$ is a maximal torus of $G$. The isomorphism class of $\psi(G, T)$ is independent of $T$, and is denoted by $\psi(G)$. The map $G \mapsto \psi(G)$ induces a natural bijection between isomorphism classes of connected reductive groups over $E$ and the isomorphism classes of root data.

We now consider a subset $\Theta$ of $G_{0}(E)=T(E)$ (resp. $\mathfrak{g}_{0}=$ Lie $T$, resp. $\left.\left(\mathfrak{g}^{*}\right)_{0}\right)$. Let $Z_{G}(\Theta)$ be the subgroup of $G$ fixing $\Theta$ pointwise under the (co)-adjoint action. Let $W=W(G, T)$ be the Weyl group of $(G, T)$ and let $z_{W}(\Theta)$ be the subgroup of $W$ fixing $\Theta$ pointwise. Let $\Phi_{\Theta}$ be the subset of $\Phi=\Phi(G, T, E)$ consisting of those $a$ such that $a(\Theta)=1$ (resp. $H_{a}^{*}(\Theta)=0$, resp. $\Theta\left(H_{a}\right)=0$ ) and let $\Phi_{\Theta}^{\vee}=\left\{a^{\vee}: a \in \Phi_{\Theta}\right\}$.

Let $\psi(G, T)=\left(X, \Phi, X^{\vee}, \Phi^{\vee}\right)$. For each $w \in W=N(T)(E) / T(E)$, let $n_{w}$ be an element of $N(T)(E)$ representing $w$.

Proposition 7.1. The group $\mathcal{Z}_{G}(\Theta)$ is generated by $T$, those $G_{a}$ such that $a \in$ $\Phi_{\Theta}$, and those $n_{w}$ such that $w \in z_{W}(\Theta)$. The identity component of $z_{G}(\Theta)$ is generated by $T$ and those $G_{a}$ such that $a \in \Phi_{\Theta}$, and is a reductive group with maximal torus $T$. The root datum of $\left(\mathcal{Z}_{G}(\Theta)^{\circ}, T\right)$ is $\left(X, \Phi_{\Theta}, X^{\vee}, \Phi_{\Theta}^{\vee}\right)$. The Weyl group $W\left(Z_{G}(\Theta)^{\circ}, T\right)=W\left(\Phi_{\Theta}\right)$ is a normal subgroup of $Z_{W}(\Theta)$. The quotient $z_{W}(\Theta) / W\left(\Phi_{\Theta}\right)$ is abelian, and is isomorphic to the group of connected components of $z_{G}(\Theta)$.

Proof. The case $\Theta \subset G_{0}(E)$ and the case $\Theta \subset \mathfrak{g}_{0}$ are well known ([St]). The case $\Theta \subset\left(\mathfrak{g}^{*}\right)_{0}$ follows from the same proof $([\mathrm{St}, 3.7])$ with the help of Lemma 6.3 , 
As a result, the list of all groups that arise as $Z_{G}(\Theta) / Z_{G}(\Theta)^{\circ}$ can be easily determined from the root datum $\psi(G, T)=\left(X, \Phi, X^{\vee}, \Phi^{\vee}\right)$. For example, to deal with the case $\Theta \subset \mathfrak{g}_{0}$, we observe that $\mathfrak{g}_{0}$ can be identified with $X^{\vee} \otimes_{\mathbb{Z}} E$. Given $\Theta \subset X^{\vee} \otimes_{\mathbb{Z}} E, z_{W}(\Theta) / W\left(\Phi_{\Theta}\right)$ can be computed without any reference to the reductive group $G$.

This observation is an essential tool in $[\mathrm{St}$, where connectedness results (see Proposition 7.2) are proved first for $\Theta \subset G_{0}(E)$, then transferred to the case $\Theta \subset \mathfrak{g}_{0}$. We now recall the result in the case $\Theta \subset \mathfrak{g}_{0}$, and transfer it to the case $\Theta \subset\left(\mathfrak{g}^{*}\right)_{0}$.

A prime number $p$ is called a torsion prime for $\left(X, \Phi, X^{\vee}, \Phi^{\vee}\right)$ if there is a $\mathbb{Z}$ closed subsystem $\Phi_{1}$ of $\Phi$ such that $X^{\vee} / \mathbb{Z}\left[\Phi_{1}^{\vee}\right]$ contains an element of order $p$. Here $\mathbb{Z}\left[\Phi_{1}^{\vee}\right]$ is the lattice contained in $\subset X^{\vee}$ and generated by $\Phi_{1}^{\vee}$.

Proposition 7.2. The centralizer $\mathcal{Z}_{G}(\Theta)$ is connected for all $\Theta \subset \mathfrak{g}_{0}$ if and only if the characteristic of $E$ is not a torsion prime of $\psi(G)$.

Proposition 7.3. The centralizer $Z_{G}(\Theta)$ is connected for all $\Theta \subset\left(\mathfrak{g}^{*}\right)_{0}$ if and only if the characteristic of $E$ is not a torsion prime of $\psi(G)^{\vee}$.

Here $\psi(G)^{\vee}$ is the dual root datum of $\psi(G)$, or equivalently, the root datum of the dual group $G^{\vee}$ of $G$. Here we take $G^{\vee}$ to be defined over $E$ (instead of over $\mathbb{C})$. The proof is immediate. In fact, let $\Theta \subset\left(\mathfrak{g}^{*}\right)_{0} \simeq X \otimes_{\mathbb{Z}} E$. Then we can identify $\Theta$ with a subset $\Theta^{\vee}$ of Lie $T^{\vee}=\left(\mathfrak{g}^{\vee}\right)_{0}$, where $T^{\vee}$ is a maximal torus of $G^{\vee}$. By Proposition 7 7.1, the component group of $Z_{G}(\Theta)$ is the same as that of $z_{G^{\vee}}\left(\Theta^{\vee}\right)$.

\section{Generic elements}

Let $G$ be a reductive group over $F$, let $\vec{G}=\left(G^{\prime}, G\right)$ be a twisted Levi sequence in $G$, let $Z^{\prime}=\mathcal{Z}\left(G^{\prime}\right)$, and let $T$ be a maximal torus of $G^{\prime}$.

We can regard $\operatorname{Lie}^{*}\left(Z^{\prime}\right)^{\circ}$ as a subspace of $\operatorname{Lie}^{*} G^{\prime}$ in a canonical way: let $V$ be the subspace of $\mathrm{Lie}^{*} G^{\prime}$ fixed by the coadjoint action of $G^{\prime}$. Each element of $V$ induces a linear function on $\operatorname{Lie}\left(Z^{\prime}\right)^{\circ} \subset \operatorname{Lie} G^{\prime}$ by restriction. This gives a linear bijection from $V$ to $\operatorname{Lie}^{*}\left(Z^{\prime}\right)^{\circ}$. We identify $\operatorname{Lie}^{*}\left(Z^{\prime}\right)^{\circ}$ with $V \subset \operatorname{Lie}^{*} G^{\prime}$.

We can also regard $\mathrm{Lie}^{*} G^{\prime}$ as a subspace of $\mathrm{Lie}^{*} G$ in a canonical way: if we consider the action of $\left(Z^{\prime}\right)^{\circ}$ on $\operatorname{Lie}^{*} G$, then the subspace fixed by $\left(Z^{\prime}\right)^{\circ}$ can be identified with $\mathrm{Lie}^{*} G^{\prime}$.

Recall that $\left(Z^{\prime}\right)^{\circ}(F)$, $\operatorname{Lie}\left(Z^{\prime}\right)^{\circ}$, $\operatorname{Lie}^{*}\left(Z^{\prime}\right)^{\circ}$ carry canonical filtrations (because $\left(Z^{\prime}\right)^{\circ}$ is a torus).

An element $X^{*}$ of $\left(\operatorname{Lie}^{*}\left(Z^{\prime}\right)^{\circ}\right)_{-r}$ is called G-generic of depth $r \in \mathbb{R}$ if two conditions GE1 and GE2 hold. The first one is very simple. The second one is quite technical, but it is implied by the first one in most cases (see Lemma 8.1). We first explain GE1 (recall that $H_{a}=d a^{\vee}(1)$ and $a^{\vee}: \mathbb{G}_{\mathrm{m}} \rightarrow T$ is the coroot of $a$ ).

GE1 $\operatorname{ord}\left(X^{*}\left(H_{a}\right)\right)=-r$ for all $a \in \Phi(G, T, \bar{F}) \backslash \Phi\left(G^{\prime}, T, \bar{F}\right)$.

To explain GE2, let $\psi(G, T)=\left(X, \Phi, X^{\vee}, \Phi^{\vee}\right)$, and let $W$ be the Weyl group of $\Phi(G, T, \bar{F})$. Recall that $\left(\operatorname{Lie}^{*} T\right) \otimes \bar{F}$ can be identified with $X \otimes_{\mathbb{Z}} \bar{F}$. Therefore, we can regard $\varpi_{r} X^{*} \in \operatorname{Lie}^{*}\left(Z^{\prime} \otimes \bar{F}\right)^{\circ} \subset \operatorname{Lie}^{*}(T \otimes \bar{F})$ as an element of $X \otimes_{\mathbb{Z}} \mathcal{O}_{\bar{F}}$, where $\varpi_{r}$ is an element of $\bar{F}^{*}$ of valuation $r$. The residue class $\tilde{X}^{*}$ of $\varpi_{r} X^{*}$ is an element of $X \otimes_{\mathbb{Z}} \bar{\kappa}$, where $\bar{\kappa}$ is the residue field of $\bar{F}$. Then $\tilde{X}^{*}$ is well defined up to a multiplicative constant in $\bar{\kappa}^{*}$. By GE1, $\Phi_{\tilde{X}^{*}}$ can be identified with $\Phi\left(G^{\prime}, T, \bar{F}\right)$.

GE2 The subgroup $z_{W}\left(\tilde{X}^{*}\right)$ of $W$ fixing $\tilde{X}^{*}$ is precisely the Weyl group of $\Phi_{\tilde{X}^{*}}=$ $\Phi\left(G^{\prime}, T, \bar{F}\right)$. 
Clearly, if the element $X^{*}$ is $G$-generic, then it is $(G \otimes E)$-generic as an element of $\operatorname{Lie}^{*}\left(Z^{\prime} \otimes E\right)_{-r}^{\circ}$ for any finite extension $E / F$. It is also easy to see that GE1 and GE2 do not depend on $T$.

Lemma 8.1. If the residual characteristic of $F$ is not a torsion prime for $\psi(G)^{\vee}$, then GE1 implies GE2.

Proof. This is an immediate consequence of Propositions 7.1 and 7.3 .

Lemma 8.2. Let $T$ be any maximal torus of $G$. For all $y \in \mathcal{B}(G, F), r \in \tilde{\mathbb{R}}$, we have

$$
\left\{\begin{array}{l}
\mathfrak{t}(F) \cap \mathfrak{g}(F)_{y, r} \subset \mathfrak{t}(F)_{r}, \\
\mathfrak{t}^{*}(F) \cap \mathfrak{g}^{*}(F)_{y, r} \subset \mathfrak{t}^{*}(F)_{r} .
\end{array}\right.
$$

Proof. We will give the proof of the first inclusion. The second one is analogous.

We first prove the lemma when $r$ is in $\operatorname{ord}\left(F^{*}\right) \otimes \mathbb{Q}$. Replacing $F$ by a finite extension if necessary, we may and do assume that $r \in \operatorname{ord}\left(F^{*}\right)$. Let $\pi_{r}$ be an element such that $\operatorname{ord}\left(\pi_{r}\right)=r$. Then $\mathfrak{t}(F)_{r}=\pi_{r} \mathfrak{t}(F)_{0}, \mathfrak{g}(F)_{y, r}=\pi_{r} \mathfrak{g}(F)_{y, 0}$. Therefore, it suffices to prove the lemma when $r=0$. But this case is obvious: an element in $\mathfrak{t}(F) \cap \mathfrak{g}(F)_{y, 0}$ is a compact element of $\mathfrak{t}(F)$, hence is in $\mathfrak{t}(F)_{0}$ (see [De] ).

If $r$ is any real number, then

$$
\left\{\begin{array}{l}
\mathfrak{g}(F)_{y, r}=\bigcap_{\substack{s<r \\
s \in \operatorname{ord}\left(F^{*}\right) \otimes \mathbb{Q}}} \mathfrak{g}(F)_{y, s}, \\
\mathfrak{t}(F)_{r}=\bigcap_{\substack{s<r \\
s \in \operatorname{ord}\left(F^{*}\right) \otimes \mathbb{Q}}} \mathfrak{t}(F)_{s} .
\end{array}\right.
$$

Therefore, the lemma in this case follows from the case $r \in \operatorname{ord}\left(F^{*}\right) \otimes \mathbb{Q}$.

If $r=s+$ for some real number $s$, then for $\epsilon>0$ small enough, we have $\mathfrak{g}(F)_{y, r}=$ $\mathfrak{t}(F)_{y, s+\epsilon}, \mathfrak{t}(F)_{r}=\mathfrak{t}(F)_{s+\epsilon}$. Therefore, the lemma in this case follows from the case $r \in \mathbb{R}$.

This proves the lemma completely.

We now assume that $\vec{G}=\left(G^{\prime}, G\right)$ is split by a tamely ramified extension $E / F$, and $T$ is a maximal torus of $G^{\prime}$ and is split over $E$. Let $y \in A(G, T, F)$. As in $\$ 2$ we consider $\mathcal{B}\left(G^{\prime}, F\right)$ as a subset of $\mathcal{B}(G, F)$ so that $y \in \mathcal{B}\left(G^{\prime}, F\right) \subset \mathcal{B}(G, F)$.

Lemma 8.3. Let $X^{*}$ be $G$-generic of depth $r, y \in \mathcal{B}\left(G^{\prime}, F\right) \subset \mathcal{B}(G, F)$. Suppose that

$$
Y_{1}^{*}, Y_{2}^{*} \in\left(\operatorname{Lie}^{*} G^{\prime}\right)(F)_{y,-r}
$$

are regular semisimple and satisfy

$$
Y_{1}^{*} \equiv Y_{2}^{*} \equiv X^{*} \quad\left(\bmod \left(\operatorname{Lie}^{*} G^{\prime}\right)(F)_{y,(-r)+}\right) .
$$

If $g \in G(F)$ is such that $\operatorname{Ad}(g) . Y_{1}^{*}=Y_{2}^{*}$, then $g \in G^{\prime}(F)$.

Proof. First observe that we can replace $F$ by any extension of $F$ without loss of generality. So we shall assume that the maximal torus $T_{i}$ fixing $Y_{i}^{*}$ is split, $i=1,2$. By multiplying $X^{*}, Y_{i}^{*}$ by a scalar of valuation $r$, we may also assume that $r=0$.

Let $\mathfrak{t}_{i}^{*}=$ Lie $^{*} T_{i}$. By Lemma $8.2 Y_{i}^{*} \in\left(\mathfrak{t}_{i}\right)_{0}^{*}$ and $Y_{i}^{*}-X^{*} \in\left(\mathfrak{t}_{i}\right)_{0+}^{*}$. 
Let $h \in G^{\prime}(F)$ be such that $h T_{1} h^{-1}=T_{2}$. Then $Y_{0}^{*}:=\operatorname{Ad}(h) . Y_{1}^{*}$ is in $\left(\mathfrak{t}_{2}\right)_{0}^{*}$, and we have

$$
Y_{0}^{*} \equiv \operatorname{Ad}(h) \cdot X^{*} \equiv X^{*} \equiv Y_{2}^{*} \quad\left(\bmod \left(\mathfrak{t}_{2}\right)_{0+}^{*}\right) .
$$

The element $w=g h^{-1}$ normalizes $T_{2}$, hence is in the Weyl group of $\left(G, T_{2}\right)$. We have $w \cdot Y_{0}^{*}=Y_{2}^{*}$.

By choosing a Chevalley basis, we can construct a Chevalley group scheme $\underline{G}$ over $\mathcal{O}_{F}$ such that the generic fiber of $\underline{G}$ is $G$, and the special fiber $G_{s}$ is reductive of the same root datum as $G$. The special fiber $\left(T_{2}\right)_{s}$ of the canonical integral model of $T_{2}$ is a maximal torus of $G_{s}$. There is a unique closed subgroup scheme $\underline{G^{\prime}}$ of $\underline{G}$ extending $G^{\prime}$, and is a Chevalley group scheme over $\mathcal{O}_{F}$ of the same root datum as $G^{\prime}$.

We can find $t \in T_{2}(F)$ such that $w t \in \underline{G}\left(\mathcal{O}_{F}\right)$. Then $w t$ reduces to an element $w^{\prime}$ of $G_{s}(\kappa)$, where $\kappa$ is the residue field of $\mathcal{O}_{F}$. We also reduce $X^{*}$ to an element $\tilde{X}^{*}$ of $\operatorname{Lie}^{*} G_{s}$. Since $\operatorname{Ad}(w t) \cdot Y_{0}^{*}=Y_{2}^{*}$, we have $\operatorname{Ad}\left(w^{\prime}\right) \cdot \tilde{X}^{*}=\tilde{X}^{*}$.

The genericity assumption precisely means that $w^{\prime}$ is in the Weyl group of $\left(G_{s}^{\prime},\left(T_{2}\right)_{s}\right)$. This implies that $w \in G^{\prime}(F)$ and hence $g=w h \in G^{\prime}(F)$.

Lemma 8.4. Suppose that $X^{*} \in\left(\operatorname{Lie}\left(Z^{\prime}\right)^{\circ}\right)_{-r}^{*}$ is G-generic of depth $r$. Let $s, t$ be real numbers such that $0<s \leq t$. The map

$$
\vec{G}(F)_{y,(s, t):(s, t+)} \rightarrow \overrightarrow{\mathfrak{g}}^{*}(F)_{y,(s-r, t-r):(s-r,(t-r)+), \quad g \mapsto g \cdot X^{*}-X^{*}}
$$

is well defined, and is a group isomorphism.

Proof. In view of Corollary 2.4 it is enough to prove this proposition under the additional hypothesis that $G, G^{\prime}$ are split. Let $\Phi=\Phi(G, T, F), \Phi^{\prime}=\Phi\left(G^{\prime}, T, F\right)$.

Choose a Chevalley system $\left\{x_{a}\right\}_{a \in \Phi}$. Recall that the Chevalley system determines a hyperspecial point $y_{0} \in A(G, T, F)$. The filtration subgroups can be described as follows (cf. [BT1, 6.2.5]):

$$
\left\{\begin{array}{l}
G_{a}(F)_{y, r}=\left\{x_{a}(u): u \in(F)_{r-a\left(y-y_{0}\right)}\right\}, \\
\mathfrak{g}_{a}(F)_{y, r}=\left\{u X_{a}: u \in(F)_{r-a\left(y-y_{0}\right)}\right\} \\
\mathfrak{g}_{a}^{*}(F)_{y, r}=\left\{u X_{a}^{*}: u \in(F)_{r-a\left(y-y_{0}\right)}\right\} .
\end{array}\right.
$$

For any $H^{*} \in \mathfrak{g}_{0}^{*}(F)$ and any $u \in F, x_{a}(u) . H^{*}-H^{*}=-u H^{*}\left(H_{a}\right) X_{a}^{*}$. Applying this to $H^{*}=X^{*}$, we see that $g \cdot X^{*}-X^{*}$ is in $\overrightarrow{\mathfrak{g}}^{*}(F)_{y,(s-r, t-r)}$ if $g$ is in $G_{a}(F)_{y,(s, t)}$ for some $a \in \Phi \cup\{0\}$. fore,

The co-adjoint action of $\vec{G}(F)_{y, 0+}$ on $\overrightarrow{\mathfrak{g}}^{*}(F)_{y,(s-r, t-r):(s-r,(t-r)+)}$ is trivial. There-

$$
\left(g_{1} g_{2}\right) \cdot X^{*}-X^{*}=g_{1}\left(g_{2} \cdot X^{*}-X^{*}\right)+\left(g_{1} \cdot X^{*}-X^{*}\right)=\left(g_{2} \cdot X^{*}-X^{*}\right)+\left(g_{1} \cdot X^{*}-X^{*}\right)
$$

modulo $\overrightarrow{\mathfrak{g}}^{*}(F)_{y,(s-r,(t-r)+)}$ for all $g_{1}, g_{2} \in \vec{G}(F)_{y,(s, t)}$ such that $\left(g_{2} . X^{*}-X^{*}\right) \in$ $\overrightarrow{\mathfrak{g}}^{*}(F)_{y,(s-r, t-r)}$. This together with [BT1, 6.4.48] shows that the map $\vec{G}(F)_{y,(s, t)} \rightarrow$ $\overrightarrow{\mathfrak{g}}^{*}(F)_{y,(s-r, t-r):(s-r,(t-r)+)}, g \mapsto g \cdot X^{*}-X^{*}$ is a well-defined group homomorphism.

Condition GE1 implies that the homomorphism is surjective with kernel $\vec{G}(F)_{y,(s, t+)}$. This completes the proof.

Lemma 8.5. Suppose that $X^{*} \in\left(\operatorname{Lie}\left(Z^{\prime}\right)^{\circ}\right)_{-r}^{*}$ is G-generic of depth $r>0$. Let $s$ be a real number strictly greater than $-r$, and let $Y^{*}$ be any element in $X^{*}+$ $\overrightarrow{\mathfrak{g}}^{*}(F)_{y,((-r)+, s)}$. Then $Y^{*}$ is conjugate to an element of $X^{*}+\overrightarrow{\mathfrak{g}}^{*}(F)_{y,((-r)+, s+)}$ by the coadjoint action of $G(F)_{y, s+r}$. 
Proof. Write $Y^{*}=Y_{1}^{*}+Y_{2}^{*}$ with $Y_{1}^{*} \in X^{*}+\left(\mathfrak{g}^{\prime}\right)^{*}(F)_{y,(-r)+}, Y_{2}^{*} \in \mathfrak{g}^{*}(F)_{y, s}$. It is enough to find $g \in G(F)_{y, s+r}$ such that $\operatorname{Ad}(g) . Y^{*}-Y_{1}^{*} \equiv 0\left(\bmod \overrightarrow{\mathfrak{g}}^{*}(F)_{y,(s, s+)}\right)$.

By the preceding lemma, we can find $g \in G(F)_{y, s+r}$ such that

$$
\operatorname{Ad}\left(g^{-1}\right) \cdot X^{*}-X^{*} \equiv Y_{2}^{*} \quad\left(\bmod \overrightarrow{\mathfrak{g}}^{*}(F)_{y,(s, s+)}\right) .
$$

Since $g \in G(F)_{y, s+r}$ and $X^{*}-Y_{1}^{*} \in \mathfrak{g}^{*}(F)_{y,(-r)+}$, we have $\operatorname{Ad}(g) \cdot\left(X^{*}-Y_{1}^{*}\right) \equiv$ $X^{*}-Y_{1}^{*}\left(\bmod \mathfrak{g}^{*}(F)_{y, s+}\right)$. Therefore,

$$
\begin{aligned}
\operatorname{Ad}(g) . Y^{*}-Y_{1}^{*} & \equiv \operatorname{Ad}(g) \cdot Y_{1}^{*}-Y_{1}^{*}+\operatorname{Ad}(g) \cdot Y_{2}^{*} \\
& \equiv \operatorname{Ad}(g) \cdot Y_{1}^{*}-Y_{1}^{*}+X^{*}-\operatorname{Ad}(g) \cdot X^{*} \\
& \equiv \operatorname{Ad}(g)\left(Y_{1}^{*}-X^{*}\right)-\left(Y_{1}^{*}-X^{*}\right) \equiv 0 \quad\left(\bmod \overrightarrow{\mathfrak{g}}^{*}(F)_{y,(s, s+)}\right) .
\end{aligned}
$$

The lemma is proved.

Lemma 8.6. With the notation and hypotheses of the preceding lemma, $Y^{*}$ is conjugate to an element of $X^{*}+\left(\mathfrak{g}^{\prime}\right)^{*}(F)_{y,(-r)+}$ by $G(F)_{y, s+r}$.

Proof. Easy from the preceding lemma.

\section{Generic Characters}

We maintain all the notation of the last section. In addition, we put $s=r / 2$ to simplify the notation.

A character $\phi$ of $G^{\prime}(F)_{y, r: r+}$ is called $G$-generic if it is realized (in the sense of (5) by an element $X^{*} \in\left(\operatorname{Lie}^{*}\left(Z^{\prime}\right)^{\circ}\right)_{-r} \subset\left(\operatorname{Lie}^{*} G^{\prime}\right)_{y,-r}$ which is $G$-generic of depth $r$.

A quasi-character $\phi$ of $G^{\prime}(F)$ is called $G$-generic (relative to $y$ ) of depth $r$ if $\phi$ is trivial on $G^{\prime}(F)_{y, r+}$, non-trivial on $G^{\prime}(F)_{y, r}$, and $\phi$ restricted to $G^{\prime}(F)_{y, r: r+}$ is $G$-generic of depth $r$ in the sense just defined.

Remark 9.1. In many cases, the point $y$ is unimportant. For example, if we have $G^{\prime}(F)_{y, r}=\left(\mathcal{D} G^{\prime}\right)(F)_{y, r} \mathcal{Z}\left(G^{\prime}\right)^{\circ}(F)_{r}$ for all $y$ and all $r$, and if $\phi$ is trivial on $\left(\mathcal{D} G^{\prime}\right)(F)$, then the $G$-genericity of $\phi$ is completely independent of $y$.

From now on, assume that $\phi$ is a $G$-generic character of $G^{\prime}(F)_{y, r}$. Put $J=$ $\left(G^{\prime}, G\right)(F)_{y,(r, s)}, J_{+}=\left(G^{\prime}, G\right)(F)_{y,(r, s+)}$. As in $4, \phi$ determines a character $\hat{\phi}$ on $J_{+}$, trivial on $\left(G^{\prime}, G\right)(F)_{y,(r+, s+)}$.

Remark 9.2. The following observation will be useful. Let $E$ be a finite extension of $F$. Since $F /(F)_{+} \rightarrow E /(E)_{+}$is injective, we can extend our additive character $\Psi: F / F_{+} \rightarrow \mathbf{C}^{*}$ to a character $\Psi^{E}$ on $E / E_{+}$. Since $\Psi$ is non-trivial on $(F)_{0}$, $\Psi^{E}$ is non-trivial on $(E)_{0}$. The character $\phi_{E}$ of $G^{\prime}(E)_{y, r: r+} \simeq \mathfrak{g}^{\prime}(E)_{y, r: r+}$ realized (via $\Psi^{E}$ ) by $X^{*} \in \operatorname{Lie}^{*}\left(Z^{\prime}\right)^{\circ} \subset\left(\operatorname{Lie}^{*}\left(Z^{\prime}\right)^{\circ}\right) \otimes E$ is simply $\phi$ when restricted to $G^{\prime}(F)_{y, r: r+}$. We now can put $J(E)=\vec{G}(E)_{y,(r, s)}$, etc. and do all the constructions over $E$. In several places, we will prove results first over a splitting field $E$, then reduce the general case to the split case.

Notice that even if $\phi$ is a character of the whole $G^{\prime}(F)$, the character $\phi_{E}$ constructed above may not be extendable to a character of $G^{\prime}(E)$.

Lemma 9.3. Let $g \in G^{\prime}(F)$. Then $\hat{\phi}\left(g^{-1} x g\right)=\hat{\phi}(x)$ for all $x \in{ }^{g \cap} J_{+}$. 
Proof. By Remark 9.2, it suffices to do this with the additional assumption that $T$ is split.

Choose an ordering on $\Phi(G, T, F)$ and define $J_{+}(+)$(resp. $\left.J_{+}(-)\right)$to be the subgroup generated by $G_{a}(F)_{y, s+}$ for all positive (resp. negative) $a \in \Phi(G, T, F)$ such that $a \notin \Phi\left(G^{\prime}, T, F\right)$.

Then we have $J_{+}=G^{\prime}(F)_{y, r} J_{+}(+) J_{+}(-)$, and every element $x$ of $J_{+}$can be decomposed as $x=x^{\prime} j_{1} j_{2}$ for unique $x^{\prime} \in G^{\prime}(F)_{y, r}, j_{1} \in J_{+}(+)$and $j_{2} \in J_{+}(-)$. The uniqueness can be deduced from [BT1, 6.4.48], but it is also a consequence of the following: Let $N(+)$ (resp. $N(-)$ ) be the subgroup generated by $G_{a}(F)$ for all positive (resp. negative) $a \in \Phi(G, T, F)$ such that $a \notin \Phi\left(G^{\prime}, T, F\right)$. Then $G^{\prime}(F) \times N(+) \times N(-) \rightarrow G(F),\left(x^{\prime}, j_{1}, j_{2}\right) \mapsto x^{\prime} j_{1} j_{2}$ is an injection (to prove this statement, just observe that $G^{\prime}(F) N(+)$ is a group, $G^{\prime}(F) N(+) \cap N(-)=\{1\}$, and $\left.G^{\prime}(F) \cap N(+)=\{1\}\right)$.

Now suppose that $x \in J_{+} \cap{ }^{g} J_{+}$. Write $x=x^{\prime} j_{1} j_{2}$ as above. Then $g^{-1} x g$ is equal to

$$
\left(g^{-1} x^{\prime} g\right)\left(g^{-1} j_{1} g\right)\left(g^{-1} j_{2} g\right)
$$

and this expression is necessarily the decomposition of $g^{-1} x g$. Therefore, we have $x^{\prime} \in{ }^{g \cap} G^{\prime}(F)_{y, r}, j_{1} \in{ }^{g \cap} J_{+}(+), j_{2} \in{ }^{g \cap} J_{+}(-)$.

Now it is clear that $\hat{\phi}(x)=\phi\left(x^{\prime}\right)$ and $\hat{\phi}\left(g^{-1} x g\right)=\phi\left(g^{-1} x^{\prime} g\right)$. It suffices to show $\phi\left(x^{\prime}\right)=\phi\left(g^{-1} x^{\prime} g\right)$, which is a consequence of [Ad, 1.6.7] and the fact that $\operatorname{Ad}(g) \cdot X^{*}=X^{*}$.

Theorem 9.4. Let $g \in G(F)$. Then $g$ intertwines $\hat{\phi}$ if and only if $g \in J G^{\prime}(F) J$.

Proof. Suppose that $g$ intertwines $\hat{\phi}$. By Lemma [5.1, we can find $Y_{1}^{*}, Y_{2}^{*}$ in $X^{*}+$ $\overrightarrow{\mathfrak{g}}^{*}(F)_{y,((-r)+,-s)}$ such that $\operatorname{Ad}(g) \cdot Y_{1}^{*}=Y_{2}^{*}$. We may assume that $Y_{1}^{*}$ and $Y_{2}^{*}$ are regular semisimple.

By Lemma 8.6 we can find $k_{1}, k_{2} \in G(F)_{y, s}$ such that

$$
Z_{i}^{*}=\operatorname{Ad}\left(k_{i}\right) \cdot Y_{i}^{*} \in X^{*}+\left(\mathfrak{g}^{\prime}\right)^{*}(F)_{y,(-r)+} .
$$

Let $g_{1}=k_{2} g k_{1}^{-1}$. Then $\operatorname{Ad}\left(g_{1}\right) \cdot Z_{1}^{*}=Z_{2}^{*}$.

By Lemma 8.3, $g_{1} \in G^{\prime}(F)$. Therefore, $g \in G(F)_{y, s} G^{\prime}(F) G(F)_{y, s}=J G^{\prime}(F) J$. This proves the "only if" part of the proposition.

Using arguments similar to those for Lemmas 4.2 and 4.3 , the reverse implication is reduced to the following statement: every $g \in G^{\prime}(F)$ intertwines $\hat{\phi}$, which is precisely Lemma 9.3

\section{Heisenberg groups and the Weil Representations}

Because an important subtlety has been neglected in most of the literature, we give a detailed discussion here.

Let $\kappa$ be a finite field of odd characteristic $p$. Let $V=(V,\langle\rangle, C$,$) be a finite-$ dimensional symplectic space over $\kappa$, where the pairing takes values in the 1dimensional vector space $C$ over $\kappa$. We allow $V$ to be zero-dimensional. The Heisenberg group $V^{\sharp}$ of $V$ is defined to be the set $V \times C$ with the group law $(v, a) \cdot(w, b)=\left(v+w, a+b+\frac{1}{2}\langle v, w\rangle\right)$. The symplectic group $\operatorname{Sp}(V)$ acts on $V^{\sharp}$ by $g \cdot(v, a)=(g \cdot v, a)$. The center of $V^{\sharp}$ is simply $\{(0, a): a \in C\}$ and is equal to the commutator subgroup of $V^{\sharp}$ when $\operatorname{dim} V>0$. Given a non-trivial character $\psi$ of $C$, there is a unique irreducible representation $\omega_{\psi}$ (called the Heisenberg representation) of $V^{\sharp}$ with central character $\psi$. It follows that $\omega_{\psi}$ extends to a projective 
representation of $\operatorname{Sp}(V) \ltimes V^{\natural}$. The theory in Ge shows that it actually extended to a representation $\omega_{\psi}$ (called the Weil representation) of $\operatorname{Sp}(V) \ltimes V^{\sharp}$ in a canonical way.

Now let $H$ be a finite $p$-group, where $p$ is an odd prime. We say that $H$ is a Heisenberg $p$-group if there exists a symplectic space $V=(V,\langle\rangle, C$,$) over \mathbb{F}_{p}$ such that $H$ is isomorphic to $V^{\sharp}$. For such a group $H$, there is a natural, non-degenerate, symplectic pairing $\langle$,$\rangle on V_{H}=H / C_{H}$ with values in $C_{H},(v, w) \mapsto v w v^{-1} w^{-1}$, where $C_{H}$ is the center of $H$. Let $H_{1}, H_{2}$ be Heisenberg $p$-groups. We say that an isomorphism $f: H_{1} \rightarrow H_{2}$ is a symplectic isomorphism if $f(\langle v, w\rangle)=\langle f(v), f(w)\rangle$ for all $v, w \in H_{1} / C_{H_{1}}$.

Let $H$ be a Heisenberg $p$-group and let $\operatorname{Sp}(H)$ be the set of symplectic isomorphisms $\sigma: H \rightarrow H$ such that $\left.\sigma\right|_{C}$ is the identity. Let $\operatorname{Sp}^{0}(H)$ be the kernel of the natural (surjective) map $\operatorname{Sp}(H) \rightarrow \operatorname{Sp}\left(V_{H}\right)$. Then $\operatorname{Sp}^{0}(H)=\left\{\sigma_{v}: v \in V_{H}\right\}$, where $\sigma_{v}(h)=h\langle h, v\rangle$. It is easy to see that $\operatorname{Sp}^{0}(H)$ is isomorphic to $V_{H}$ by $\sigma_{v} \mapsto v$.

Let $(V,\langle\rangle, C)=,\left(V_{H},\langle\rangle,, C_{H}\right)$. Call an isomorphism $j: H \rightarrow V^{\sharp}$ special if $\left.j\right|_{C}$ is the identity and $j$ induces the identity map from $V=V_{H} / C_{H}$ to $V^{\sharp} / C=V$. The set of special isomorphisms is a principal homogeneous space of $\operatorname{Sp}^{0}(H) \simeq V$.

Now let $K$ be a group acting on $H$ by automorphisms. Suppose that $K$ acts trivially on $C$, and $\langle g . v, g . w\rangle=\langle v, w\rangle$ for all $g \in K, v, w \in H$. We then have a homomorphism $f: K \rightarrow \operatorname{Sp}(H)$.

In the literature, people often write: then we have homomorphisms $K \rightarrow \operatorname{Sp}(V)$ and $K \ltimes H \rightarrow \mathrm{Sp}(V) \ltimes H$, and we can pull back the Weil representation (after choosing a central character).

This is not true. For an abstract Heisenberg $p$-group, there is no natural way of making $\operatorname{Sp}(V)$ act on $H$. To get such an action, we need to choose a splitting $s: \mathrm{Sp}(V) \rightarrow \mathrm{Sp}(H)$ of the exact sequence

$$
1 \rightarrow \mathrm{Sp}^{0}(H) \rightarrow \mathrm{Sp}(H) \rightarrow \mathrm{Sp}(V) \rightarrow 1
$$

In order to pull back the Weil representation, we need more: we need to choose a splitting $s$ for which there exists a special isomorphism $j: H \rightarrow V^{\sharp}$ which transforms $s$ to the splitting of $\operatorname{Sp}\left(V^{\sharp}\right) \rightarrow \operatorname{Sp}(V)$ defined by the natural action of $\operatorname{Sp}(V)$ on $V^{\sharp}$. The set of all such splittings is a principal homogeneous space of $\operatorname{Sp}^{0}(H) \simeq V$.

In order to get a homomorphism $K \ltimes H \rightarrow \operatorname{Sp}(V) \ltimes H$, the composition $K \rightarrow$ $\mathrm{Sp}(H) \rightarrow \operatorname{Sp}(V)$ followed by $s: \operatorname{Sp}(V) \rightarrow \operatorname{Sp}(H)$ has to be identical to $f: K \rightarrow$ $\operatorname{Sp}(H)$. This is clearly impossible if $f(K) \cap \operatorname{Sp}^{0}(H) \neq\{1\}$. If $f(K) \cap \operatorname{Sp}^{0}(H)=\{1\}$, there is still an obstruction for finding an allowable $s$, and that obstruction is represented by a class in $H^{1}\left(f(K), \mathrm{Sp}^{0}(H)\right)=H^{1}(f(K), V)$. This obstruction class can be non-trivial.

Even if all the obstructions vanish, we still can't say that the pull back of $\omega_{\psi}$ is "canonical". In general the isomorphism class of the pull back still depends on the choice of the special isomorphism mentioned above.

Therefore, we introduce the following notion: let $H$ be an abstract Heisenberg p-group with center $C, V=H / C$, and let $K$ be another group. A symplectic action of $K$ on $H$ is a pair $(f, j)$ such that $f: K \rightarrow \operatorname{Sp}(H)$ is a homomorphism, $j: H \rightarrow V^{\sharp}$ is a special isomorphism, and the map $\left.K \rightarrow \operatorname{Sp} V^{\sharp}\right), g \rightarrow j \circ f(g) \circ j^{-1}$ has image in $\left.\operatorname{Sp}(V) \subset \operatorname{Sp} V^{\sharp}\right)$. In this case, $K \ltimes H \rightarrow \operatorname{Sp}(V) \ltimes V^{\sharp}, g \ltimes h \mapsto j \circ f(g) \circ j^{-1} \ltimes j(h)$ is a group homomorphism. We define the Weil representation of $K \ltimes H$ to be the pull back of $\omega_{\psi}$.

We now give a way to recognize Heisenberg $p$-groups and symplectic actions. 
Let $H$ be a finite $p$-group with center $C$ such that $[H, H] \subset C$, and $C$ is of order $p$. We also assume that the natural symplectic pairing $\langle$,$\rangle on V=H / C$ with values in $C$ is non-degenerate. Let $\pi$ be the natural map $H \rightarrow V$. Suppose that there are subgroups $W_{1}, W_{2}$ of $H$ such that $W_{1} \cap C=W_{2} \cap C=\{1\}$, and $\pi\left(W_{1}\right)$ and $\pi\left(W_{2}\right)$ form a complete polarization of $V$.

It follows that every element of $H$ can be expressed uniquely as $w_{1} w_{2} c$, with $w_{1} \in W_{1}, w_{2} \in W_{2}, c \in C$. Define $j=j_{W_{1}, W_{2}}: H \rightarrow V^{\sharp}$ by

$$
j\left(w_{1} w_{2} c\right)=\left(\pi\left(w_{1}\right)+\pi\left(w_{2}\right), c+\frac{1}{2}\left\langle\pi\left(w_{1}\right), \pi\left(w_{2}\right)\right\rangle\right) .
$$

Lemma 10.1. The group $H$ is a Heisenberg p-group and the map $j$ is a special isomorphism.

Proof. We have $\left(w_{1} w_{2} c\right) \cdot\left(w_{1}^{\prime} w_{2}^{\prime} c^{\prime}\right)=\left(w_{1} w_{1}^{\prime}\right)\left(w_{2} w_{2}^{\prime}\right)\left(c+c^{\prime}-\left\langle w_{1}^{\prime}, w_{2}\right\rangle\right)$. It is easy to check that $j$ is a group isomorphism. It is clear that $\left.j\right|_{C}$ is trivial and $j$ induces the identity $V=H / C \rightarrow V=V^{\sharp} / C$.

Lemma 10.2. Maintain the above situation. Suppose that $K$ is a group and $f$ : $K \rightarrow \operatorname{Sp}(H)$ is a homomorphism. If $f(g) . W_{1} \subset W_{1}$ and $f(g) . W_{2} \subset W_{2}$ for all $g \in K$, then $(f, j)$ is a symplectic action.

Proof. Let $\bar{f}$ be the composite of $f: K \rightarrow \operatorname{Sp}(H)$ and $\operatorname{Sp}(H) \rightarrow \operatorname{Sp}(V)$. We have to show that for all $w_{1} \in W_{1}, w_{2} \in W_{2}, c \in C$,

$$
\begin{array}{r}
j \circ f(g) \circ j^{-1}\left(\pi\left(w_{1}\right)+\pi\left(w_{2}\right), c+\frac{1}{2}\left\langle\pi\left(w_{1}\right), \pi\left(w_{2}\right)\right\rangle\right) \\
=\left(\bar{f}(g)\left(\pi\left(w_{1}\right)+\pi\left(w_{2}\right)\right), c+\frac{1}{2}\left\langle\pi\left(w_{1}\right), \pi\left(w_{2}\right)\right\rangle\right) .
\end{array}
$$

By assumption, $\bar{f}(g)\left(\pi\left(w_{i}\right)\right)=\pi\left(f(g) w_{i}\right), i=1,2$. Therefore,

$$
j\left(f(g)\left(w_{1}\right) f(g)\left(w_{2}\right) f(g)(c)\right)=\left(\bar{f}(g)\left(\pi\left(w_{1}\right)+\pi\left(w_{2}\right)\right), c+\frac{1}{2}\left\langle\pi\left(w_{1}\right), \pi\left(w_{2}\right)\right\rangle\right) .
$$

Therefore, the formula we want to prove is equivalent to

$$
f(g)\left(w_{1} w_{2} c\right)=f(g)\left(w_{1}\right) f(g)\left(w_{2}\right) f(g)(c),
$$

which is obviously true.

Lemma 10.3. Let $K$ be a group, let $H$ be a Heisenberg p-group with center $C$, and let $(f, j)$ be a symplectic action of $K$ on $H$. Let $V_{1}$ be a non-degenerate subspace of $V=H / C$, and let $H_{1}$ be the preimage of $V_{1}$ in $H$. Then $H_{1}$ is a Heisenberg p-group. Suppose that $K_{1}$ is a subgroup of $K$ such that $K_{1} \cdot H_{1} \subset H_{1}$. Define $f_{1}: K_{1} \rightarrow \operatorname{Sp}\left(H_{1}\right)$ by $f_{1}\left(g_{1}\right)=\left.f\left(g_{1}\right)\right|_{H_{1}}$ and $j_{1}: H_{1} \rightarrow V_{1}^{\sharp}$ by $\left.j\right|_{H_{1}}$. Then $\left(f_{1}, j_{1}\right)$ is a symplectic action of $K_{1}$ on $H_{1}$.

Proof. It is clear that $j_{1}\left(H_{1}\right)=j\left(H_{1}\right)$ is the inverse image of $V_{1}$ in $V^{\sharp}$, and is equal to $V_{1}^{\sharp}$ by definition. Therefore, $H_{1}$ is a Heisenberg $p$-group and $j_{1}$ is a special isomorphism.

For any $g \in K_{1}, j_{1} \circ f_{1}(g) \circ j_{1}^{-1}$ is the restriction of $j \circ f(g) \circ j^{-1}$ to $H_{1}$. Therefore, it is of the form $(v, c) \mapsto(\bar{f}(g) \cdot v, c)$ for some $\bar{f}(g) \in \mathrm{Sp}(V)$. By assumption, $\bar{f}(g) . V_{1} \subset V_{1}$ and hence $\left.\bar{f}(g)\right|_{V_{1}} \in \operatorname{Sp}\left(V_{1}\right)$. This shows that $\left(f_{1}, j_{1}\right)$ is a symplectic action. 


\section{WEIL REPRESENTATIONS ARISING FROM GENERIC CHARACTERS}

We continue with the notation and assumptions from \$9 From now on, we assume that the residual characteristic $p$ of $F$ is odd.

Let $N=\operatorname{ker} \hat{\phi}$. We define $\langle a, b\rangle=\hat{\phi}\left(a b a^{-1} b^{-1}\right)$ for $a, b \in J$. This is well defined because $[J, J] \subset J_{+}$. Since $\left[J, J_{+}\right] \subset \vec{G}(F)_{y,(r+, s+)} \subset N$, we easily see that $\langle a, b\rangle$ depends on $a J_{+}$and $b J_{+}$only (the statement about the commutator group is a special case of Lemma 4.21). It is also easy to see that the induced function $\langle$,$\rangle on$ $J / J_{+} \times J / J_{+}$is bi-additive and symplectic (in the sense that $\langle a, a\rangle=0$ for all $a$ ).

Lemma 11.1. The pairing $\langle$,$\rangle is non-degenerate on J / J_{+}$.

Proof. Recall that $J / J_{+}$is naturally isomorphic to $\overrightarrow{\mathfrak{g}}(F)_{y,(r, s):(r, s+)}$. We may consider the pairing $\langle$,$\rangle as being defined on the latter group. From the definitions, it$ is easy to see that if $A, B \in \overrightarrow{\mathfrak{g}}(F)_{y,(r, s)}$, then $\langle A, B\rangle=\Psi \circ X^{*}([A, B])$.

The function $B \mapsto X^{*}([A, B])$ on $\mathfrak{g}(F)$ is linear, hence is an element of $\mathfrak{g}^{*}$. We denote this element by $\left[X^{*}, A\right]$. So we have $\left[X^{*}, A\right](B)=X^{*}([A, B])$.

Now suppose that $\Psi \circ\left[X^{*}, A\right](B)=1$ for all $B \in \overrightarrow{\mathfrak{g}}(F)_{y,(r, s)}$. Then $\left[X^{*}, A\right] \in$ $\overrightarrow{\mathfrak{g}}^{*}(F)_{y,((-r)+,(-s)+)}$ by duality.

By the next lemma, this implies that $A \in \overrightarrow{\mathfrak{g}}(F)_{y,(0+, s+)}$. Therefore,

$$
A \in \overrightarrow{\mathfrak{g}}(F)_{y,(0+, s+)} \cap \overrightarrow{\mathfrak{g}}(F)_{y,(r, s)}=\overrightarrow{\mathfrak{g}}(F)_{y,(r, s+)} .
$$

This proves the non-degeneracy.

Lemma 11.2. For any real numbers $u, v$, the map $A \mapsto\left[X^{*}, A\right]$ induces an isomorphism

$$
\overrightarrow{\mathfrak{g}}(F)_{y,(u, v):(u, v+)} \simeq \overrightarrow{\mathfrak{g}}^{*}(F)_{y,(u-r, v-r):(u-r,(v-r)+) .}
$$

Proof. This is similar to and easier than Lemma 8.4

Observe that if $g \in G^{\prime}(F) \cap G(F)_{[y]}$, then $g$ normalizes $J$ and $J_{+}$, and we have

Lemma 11.3. For any $g \in G^{\prime}(F) \cap G(F)_{[y]}$, we have $\langle g a, g b\rangle=\langle a, b\rangle$ for all $a, b \in J$, and $\hat{\phi}\left(g_{a g}{ }^{-1}\right)=\hat{\phi}(a)$ for all $a \in J_{+}$.

Proof. It suffices to show that

$$
\Psi \circ X^{*}([\operatorname{Ad}(g) \cdot A, \operatorname{Ad}(g) \cdot B])=\Psi \circ X^{*}([A, B]) \quad \text { for } A, B \in \overrightarrow{\mathfrak{g}}(F)_{y,(r, s)},
$$

and

$$
\Psi \circ X^{*}(\operatorname{Ad}(g) \cdot A)=\Psi \circ X^{*}(A) \quad \text { for } A \in \overrightarrow{\mathfrak{g}}(F)_{y,(r, s+)} .
$$

Both are immediate from the fact that $X^{*} \circ \operatorname{Ad}(g)=\operatorname{Ad}\left(g^{-1}\right) \cdot X^{*}=X^{*}$.

Notice that Lemma 11.1 implies that the center of $J / N$ is $J_{+} / N$, which is also the commutator subgroup of $J / N$ if $J \neq J_{+}$.

Proposition 11.4. Suppose that the residual characteristic $p$ of $F$ is odd. Then $J / N$ is a Heisenberg $p$-group, and there is a canonical special isomorphism $j$ : $J / N \rightarrow\left(J / J_{+}\right)^{\sharp}$. Let $f: G^{\prime}(F) \cap G(F)_{[y]} \rightarrow \operatorname{Sp}(J / N)$ be the homomorphism induced by the conjugate action of $G^{\prime}(F) \cap G(F)_{[y]}$ on $J$. Then $(f, j)$ is a symplectic action on $J / N$. 
Proof. We will first prove the proposition in the case that $G$ and $G^{\prime}$ are split over $F$.

Let $S \subset G^{\prime}$ be a maximal torus which is split over $F$ and such that $y \in$ $A\left(G^{\prime}, S, F\right)$. We choose an ordering on $\Phi(G, S, F)$ and define $J(+)$ (resp. $J(-)$ ) to be the subgroup of $J$ generated by $G_{a}(F)_{y, s}$ for all positive (resp. negative) $a \in \Phi(G, S, F)$ such that $a \notin \Phi\left(G^{\prime}, S, F\right)$.

It is easy to show that $J(+) N \cap J_{+}=N$. Using Bruhat-Tits theory (BT1. 6.4.44]), we can show that

$$
[J(+) N, J(+) N] \subset\left[J(+) J_{+}, J(+) J_{+}\right] \subset\left(G^{\prime}, G\right)(F)_{y,(r+, s+)} \subset N .
$$

Therefore, $W(+)=J(+) N / N$ maps injectively into $J / J_{+}$, and the image $\bar{W}(+)$ is a totally isotropic subspace with respect to $\langle$,$\rangle . All these statements apply to$ $J(-)$ as well. Since $\bar{W}(+)+\bar{W}(-)=J / J_{+}$, and both $\bar{W}(+)$ and $\bar{W}(-)$ are totally isotropic, we conclude that $\bar{W}(+)$ and $\bar{W}(-)$ form a complete polarization of $J / J_{+}$. By Lemma 10.1 $J / N$ is a Heisenberg $p$-group, and there is a special isomorphism $j=j_{W(+), W(-)}$ from $J / N$ to $\left(J / J_{+}\right)^{\sharp}$.

It is clear that for all $g \in G^{\prime}(F) \cap G(F)_{[y]}$, we have $g J(+) N g^{-1}=J(+) N$ and $g J(-) N g^{-1}=J(-) N$. By Lemma 10.2, $(f, j)$ is a symplectic action.

It remains to show that the special isomorphism doesn't depend on the choice of $S$ and the choice of ordering. Observe that for any $a \in \Phi(G, S, F) \backslash \Phi\left(G^{\prime}, S, F\right)$ and any $u \in G_{a}(F)_{y, s}, j(u)$ is simply $\left(u J_{+}, 0\right) \in\left(J / J_{+}\right)^{\sharp}$. Since $\{u N: u \in$ $G_{a}(F)_{y, s}$ for some $\left.a \in \Phi(G, S, F) \backslash \Phi\left(G^{\prime}, S, F\right)\right\}$ together with $J_{+} / N$ generate $J / N$, we see that $j$ doesn't depend on the choice of ordering.

Let $B$ be the Borel subgroup of $G$ determined by the chosen ordering. Let $P$ be the parabolic subgroup containing $B$ such that $G^{\prime}$ is a Levi factor of $P$. Let $U$ be the unipotent radical of $P$. Then $U(F) \cap J=J(+)$. If we choose a different torus $S_{1}$, then there is an ordering on $\Phi\left(G, S_{1}, F\right)$ such that $U_{1}(F)=U(F)$ (where $B_{1}, P_{1}, U_{1}$ are the obvious counterparts of $\left.B, P, U\right)$. Therefore, we obtain the same $W(+)$ and $W(-)$ if we use $S_{1}$ in the construction. This shows that $j$ doesn't depend on the choice of the torus $S$.

The proof of the split case is now complete. We now reduce the general case to the split case.

Take $E$ to be a tamely ramified splitting field for $T$. From the split case, $J(E) / N(E)$ is a Heisenberg p-group, and there is a canonical special isomorphism $j: J(E) / N(E) \rightarrow\left(J(E) / J_{+}(E)\right)^{\sharp}$. Let $\iota$ be the injection $J / N \rightarrow J(E) / N(E)$. The image $\iota(J / N)$ in $J(E) / J_{+}(E)$ is isomorphic to $J / J_{+}$, and hence is a non-degenerate subspace. The action of $G^{\prime}(F) \cap G(F)_{[y]} \subset G^{\prime}(E) \cap G(E)_{[y]}$ stabilizes $\iota(J / N)$. By Lemma 10.3, we get a symplectic action $(f, j)$ of $G^{\prime}(F) \cap G(F)_{[y]}$ on the Heisenberg $p$-group $J / N$.

It is easy to check that this construction is independent of the choice of the splitting field $E$. This completes the proof of the general case.

Theorem 11.5. Let $K=G^{\prime}(F) \cap G(F)_{[y]}$ and let $\tilde{\phi}$ be the representation of $K \ltimes J$ which is the pull back of the Weil representation of $\operatorname{Sp}\left(J / J_{+}\right) \ltimes(J / N)$ via the symplectic action given by the preceding proposition. Then (i) the restriction of $\tilde{\phi}$ on $J_{+}=1 \ltimes J_{+}$is $\hat{\phi}$-isotypic; and (ii) the restriction of $\tilde{\phi}$ on $G^{\prime}(F)_{y, 0+} \ltimes 1$ is 1-isotypic.

Proof. (i) is clear from the definition of the Heisenberg representation. To prove (ii), it suffices to show that the action (by conjugation) of $G^{\prime}(F)_{y, 0+}$ on $J / J_{+}$is 
trivial. This follows from the fact $\left[G^{\prime}(F)_{y, 0+}, J\right] \subset J_{+}$, which is an easy consequence of [BT1, 6.4.44].

\section{Calculating intertwining: Counting arguments}

We begin with two general facts about Heisenberg representations.

Let $\kappa$ be a finite field of odd characteristic $p$. Let $V=(V,\langle\rangle, C$,$) be a finite-$ dimensional symplectic space over $\kappa$, let $\psi$ be a non-trivial character of $C$, and let $\omega=\omega_{V}$ be the Heisenberg representation of $V^{\sharp}$ with central character $\psi$.

Lemma 12.1. The character of $\omega$ is given by

$$
\operatorname{Tr} \omega(u)= \begin{cases}(\# V)^{1 / 2} \psi(u) & \text { if } u \in C, \\ 0 & \text { if } u \notin C .\end{cases}
$$

Proof. All we need to know are (i) the dimension of the space of $\omega$ is $(\# V)^{1 / 2}$, (ii) the central character of $\omega$ is $\psi$, and (iii) $\omega$ is irreducible.

(i) and (ii) imply $\operatorname{Tr} \omega(u)=(\# V)^{1 / 2} \psi(u)$ if $u \in C$. (iii) implies $\sum_{u \in V^{\sharp}}|\operatorname{Tr} \omega(u)|^{2}$ $=(\# V)(\# \kappa)$. But $\sum_{u \in C}|\operatorname{Tr} \omega(u)|^{2}=(\# V)(\# \kappa)$, and this forces $\operatorname{Tr} \omega(u)=0$ for $u \notin C$.

Let $U$ be a subspace of $V$, and let $U^{\sharp}$ be the preimage of $U$ in the projection $V^{\sharp} \rightarrow V$. We will analyze $\left.\omega_{V}\right|_{U^{\sharp}}$. Let $U_{0}=\{u \in U:\langle u, U\rangle=0\}$. Then $U / U_{0}$ is a non-degenerate symplectic space, and we can find a non-degenerate subspace $V_{1}$ such that $U=U_{0} \oplus V_{1}$ (in general $V_{1}$ is not unique). Then $U^{\sharp}=U_{0}^{\sharp} V_{1}^{\sharp}$. We denote by $\left.\omega_{V}\right|_{U_{0}^{\sharp} \times V_{1}^{\sharp}}$ the inflation of $\left.\omega_{V}\right|_{U^{\sharp}}$ to $U_{0}^{\sharp} \times V_{1}^{\sharp}$.

Lemma 12.2. Let $N_{0}=\left(\#\left(V / V_{1}\right)\right)^{1 / 2} / \# U_{0}$. Then $\left.\omega_{V}\right|_{U_{0}^{\sharp} \times V_{1}^{\sharp}}$ decomposes into a sum over irreducible representations $\chi \otimes \omega_{V_{1}}$, each occurring with multiplicity $N_{0}$, where $\chi$ runs over all linear characters of $U_{0}^{\sharp}$ extending $\psi$, and $\omega_{V_{1}}$ is the Heisenberg representation of $V_{1}^{\sharp}$ with central character $\psi$.

Proof. By the preceding lemma,

$$
\left.\operatorname{Tr} \omega_{V}\right|_{U_{0}^{\sharp} \times V_{1}^{\sharp}}\left(\left(u_{0}, c_{0}\right) \times\left(v_{1}, c_{1}\right)\right)=N_{0}\left(\operatorname{Tr} \operatorname{reg}\left(u_{0}\right)\right) \psi\left(c_{0}\right)\left(\operatorname{Tr} \omega_{V_{1}}\left(v_{1}, c_{1}\right)\right),
$$

where reg is the regular representation of $U_{0}$. The lemma follows immediately.

Now we return to the notation and assumptions of the preceding section. As in Theorem 11.5 we put $K=G^{\prime}(F) \cap G(F)_{[y]}$ and let $\tilde{\phi}$ be the representation of $K \ltimes J$ which is the pull back of the Weil representation of $\operatorname{Sp}\left(J / J_{+}\right) \ltimes(J / N)$ via the symplectic action given by Proposition 11.4. Our goal is to prove the following proposition.

Proposition 12.3. For all $g \in G^{\prime}(F)$, we have $\operatorname{dim} I_{g}\left(\left.\tilde{\phi}\right|_{J}\right)=1$.

We will give a few counting arguments in this section and reduce this proposition to the existence of a certain decomposition. The required decomposition will be constructed in the next section.

Lemma 12.4. For any $g \in G^{\prime}(F), \operatorname{dim} I_{g}\left(\left.\tilde{\phi}\right|_{J}\right)=\left[J: J_{+}\right] /\left[{ }^{g \cap} J:{ }^{g \cap} J_{+}\right]$. In particular, $g$ always intertwines $\left.\tilde{\phi}\right|_{J}$. 
Proof. Let $p^{2 n}=\left[J: J_{+}\right]$. By Lemma 12.1, the character $\operatorname{Tr} \tilde{\phi} \mid g \cap J(x)$ is non-zero precisely when $x \in\left({ }^{g \cap} J\right) \cap J_{+}=J_{+} \cap\left({ }^{g} J\right)$. Similarly, $\left.\operatorname{Tr}{ }^{g} \tilde{\phi}\right|_{g \cap J}(x)$ is non-zero pre-

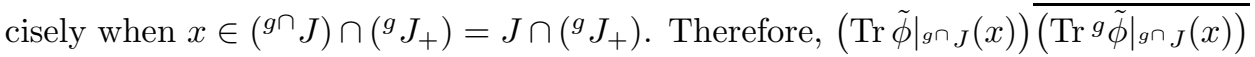
is non-zero precisely when $x \in J_{+} \cap\left({ }^{g} J_{+}\right)$, and in this case it takes the value

$$
p^{n} \hat{\phi}(x) \overline{p^{n} \hat{\phi}\left(g^{-1} x g\right)}=p^{2 n}
$$

by Lemma 9.3 From character theory, the intertwining number is equal to $p^{2 n} /\left[{ }^{g \cap} J:{ }^{g \cap} J_{+}\right]$.

Lemma 12.5. Let $g \in G^{\prime}(F)$. Then $\left[\left({ }^{g \cap} J\right) J_{+}:{ }^{g \cap} J\right]=\left[J_{+}: J_{+} \cap\left({ }^{g} J\right)\right]$ and $\left[\left(J \cap\left({ }^{g} J_{+}\right)\right) J_{+}: J_{+}\right]=\left[J \cap\left({ }^{g} J_{+}\right):{ }^{g \cap} J_{+}\right]$.

Proof. In this proof, if $B$ is a subgroup of $G^{\prime}(F)$ and $A$ is a subset of $G(F)$ such that $A B=A$, then we denote by $[A: B]$ the number of distinct cosets $a B$ in $A$. Recall the elementary index equality $[A B: B]=[A: A \cap B]$, valid for any two subgroups $A, B$ of $G(E)$.

Apply the equality with $A=\left({ }^{g \cap} J\right) J_{+}, B={ }^{g} J$ (note that $A$ is a group because $g \cap J$ is a subgroup of $J$ and $J_{+}$is a normal subgroup of $\left.J\right)$. We get $\left[\left({ }^{g \cap} J\right) J_{+}\left({ }^{g} J\right)\right.$ : $\left.{ }^{g} J\right]=\left[\left({ }^{g \cap} J\right) J_{+}:{ }^{g \cap} J\right]$. Observe that

$$
\left({ }^{g \cap} J\right) J_{+}\left({ }^{g} J\right)=J_{+}\left({ }^{g \cap} J\right)\left({ }^{g} J\right)=J_{+}\left({ }^{g} J\right) .
$$

Therefore, $\left[\left({ }^{g \cap} J\right) J_{+}\left({ }^{g} J\right):{ }^{g} J\right]=\left[J_{+}\left({ }^{g} J\right):{ }^{g} J\right]=\left[J_{+}: J_{+} \cap\left({ }^{g} J\right)\right]$. This proves the first part of the lemma. The second part is a straightforward application of the index equality.

Lemma 12.6. Fix $g \in G^{\prime}(F)$. The following are equivalent:

(i) $\operatorname{dim} I_{g}\left(\left.\tilde{\phi}\right|_{J}\right)=1$;

(ii) $\left[J: J_{+}\right]=\left[{ }^{g \cap} J:{ }^{g \cap} J_{+}\right]$;

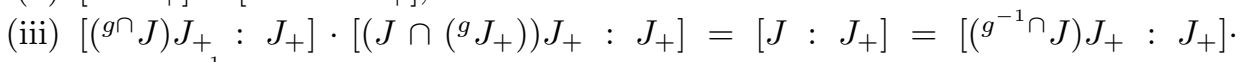
$\left[\left(J \cap\left(g^{-1} J_{+}\right)\right) J_{+}: J_{+}\right]$.

Proof. The equivalence of (i) and (ii) is immediate from Lemma 12.4

Now consider the diagram of inclusions

$$
\begin{array}{ccccc}
(g \cap J) J_{+} & \stackrel{p^{c}}{\supset} g \cap J & p^{m} & g \cap J_{+} \\
\bigcup p^{m-a-b} & & & \bigcap p^{b} \\
\left(J \cap\left(g J_{+}\right)\right) J_{+} & \stackrel{p^{a}}{\supset} & J_{+} & \stackrel{p^{c}}{\supset} & J_{+} \cap\left({ }^{g} J\right)
\end{array}
$$

The number by each inclusion symbol indicates the index. We have applied Lemma 12.5 to label two of the indices by the same number $p^{c}$.

Let $p^{2 n}=\left[J: J_{+}\right]$. We will show that $p^{m+a-b} \leq p^{2 n}$ in the remark following this proof. We now assume this inequality holds. Suppose that (ii) holds, i.e. $m=2 n$. Then $a \leq b$, i.e. $\left[J \cap\left({ }^{g} J_{+}\right):{ }^{g \cap} J_{+}\right] \leq\left[J_{+} \cap\left({ }^{g} J\right):{ }^{g \cap} J_{+}\right]$(we are applying the second part of Lemma 12.5) . However, (ii) also implies that $p^{2 n}=\left[g^{-1} \cap J: g^{-1} \cap J_{+}\right]$. So we have $\left[J \cap\left(g^{-1} J_{+}\right): g^{-1} \cap J_{+}\right] \leq\left[J_{+} \cap\left(g^{-1} J\right):{ }^{g^{-1} \cap} J_{+}\right]$. Applying the inner automorphism $x \mapsto g x g^{-1}$, we get $\left[{ }^{g} J \cap J_{+}:{ }^{g \cap} J_{+}\right] \leq\left[{ }^{g} J_{+} \cap J:{ }^{g \cap} J_{+}\right]$, i.e. $b \leq a$. We conclude that $a=b$. The two equalities $a=b$ and $m=2 n$ imply (iii).

Conversely, let's assume that (iii) holds. Now condition (iii) says precisely that $m+a-b=2 n$ and $m+b-a=2 n$. Therefore, we conclude $b=a$ and $m=2 n$, hence (ii). 
Remark 12.7. We can give a direct proof that (iii) implies (i). Let $U$ (resp. $U_{0}$ ) be the image of $J \cap\left({ }^{g} J\right)$ (resp. $\left.J \cap\left({ }^{g} J_{+}\right)\right)$in $V=J / J_{+}$. We claim that $U_{0} \perp U$. In fact, if $a \in J \cap\left({ }^{g} J\right), b \in J \cap\left({ }^{g} J_{+}\right)$, then $a b a^{-1} b^{-1} \in J_{+} \cap{ }^{g}\left[J, J_{+}\right]$and $\hat{\phi}\left(a b a^{-1} b^{-1}\right)=$ $\hat{\phi}\left(g^{-1}\left(a b a^{-1} b^{-1}\right) g\right)=1$ by Lemma 9.3 . In particular, $U_{0}$ is totally isotropic, and $\operatorname{dim} U_{0}+\operatorname{dim} U \leq 2 n$, i.e. $a+(m-b) \leq 2 n$. This justifies the statement used in the proof of equivalence of (ii) and (iii).

Remember that $\operatorname{dim} U_{0}+\operatorname{dim}\left(U_{0}\right)^{\perp}=\operatorname{dim} V$. Therefore, the first equality of (iii) is equivalent to $U=\left(U_{0}\right)^{\perp}$. We now assume that this is the case. By Lemma 12.2 this implies that $\left.\tilde{\phi}\right|_{g \cap J}$ decomposes into the direct sum of $d=\# U_{0}$ distinct irreducible representations $\pi_{1}, \ldots, \pi_{d}$. We assume that $\pi_{1}$ is the unique irreducible subrepresentation which is $\left(\left.{ }^{g} \hat{\phi}\right|_{J \cap\left({ }^{g} J_{+}\right)}\right)$-isotypic.

Let $f$ be a non-zero element in $I_{g}\left(\left.\tilde{\phi}\right|_{J}\right)$. The image of $f$ is a direct sum of a collection of $\pi_{i}$ 's. Let $v$ be a non-zero element in the space of $\pi_{i}$ such that $v$ is in the image of $f$, say $v=f\left(v^{\bullet}\right)$. Then since $v^{\bullet}$ is a ${ }^{g} \hat{\phi}$-eigenvector under the action of ${ }^{g} J_{+} \cap J, v$ must be the same kind of eigenvector. So we must have $i=1$.

Similarly, $\left.{ }^{g} \tilde{\phi}\right|_{g \cap J}$ decomposes into distinct representations $\pi_{1}^{\bullet}, \ldots, \pi_{d}^{\bullet}$. We assume that $\pi_{1}^{\bullet}$ is the unique irreducible subrepresentation on which $J_{+} \cap\left({ }^{g} J\right)$ acts $\hat{\phi}$-isotypically. The second equality in (iii) implies that if $v^{\bullet}$ is in the space of $\pi_{i}^{\bullet}$ such that $f\left(v^{\bullet}\right) \neq 0$, then $i=1$. Therefore, the homomorphism $f$ determines, and is determined by, an element of $\operatorname{Hom}_{g \cap J}\left(\pi_{1}^{\bullet}, \pi_{1}\right)$. We conclude that $\operatorname{dim} I_{g}\left(\left.\tilde{\phi}\right|_{J}\right)$ is at most one, hence is exactly one, by Lemma 12.4 This finishes our direct proof of (iii) implying (i).

This proof allows us to see the intertwining explicitly. Basically, both $\left.{ }^{g} \tilde{\phi}\right|_{g \cap J}$ and $\left.\tilde{\phi}\right|_{g \cap J}$ contain a unique irreducible subrepresentation which is the inflation of the Heisenberg representation of $\left(U / U_{0}\right)^{\sharp}$, and the only possible non-zero intertwining operators are isomorphisms between these two subrepresentations. This argument will be considerably refined in $\$ 14$.

Lemma 12.8. Fix $g \in G^{\prime}(F)$. Let $J_{1}, J_{2}, J_{3}$ be three subgroups of $J$, and let $V_{1}, V_{2}, V_{3}$ be their images in $V=J / J_{+}$. Suppose

(a) $V=V_{1} \oplus V_{2} \oplus V_{3}$;

(b) $V_{1} \perp V_{2}, V_{2} \perp V_{3}$;

(c) $V_{1}$ and $V_{3}$ are totally isotropic;

(d) $V_{1}=\left(J \cap\left({ }^{g} J_{+}\right)\right) J_{+} / J_{+}, V_{1} \oplus V_{2}=\left({ }^{g \cap} J\right) J_{+} / J_{+}$.

Then $\left[\left({ }^{g \cap} J\right) J_{+}: J_{+}\right] \cdot\left[\left(J \cap\left({ }^{g} J_{+}\right)\right) J_{+}: J_{+}\right]=\left[J: J_{+}\right]$.

Proof. By (d), we only have to show that $\operatorname{dim}\left(V_{1} \oplus V_{2}\right)+\operatorname{dim} V_{1}=\operatorname{dim} V$ (here $\operatorname{dim}=$ $\operatorname{dim}_{\mathbb{F}_{p}}$ ). By (a), this is equivalent to $\operatorname{dim} V_{1}=\operatorname{dim} V_{3}$. By (b), $V_{1} \oplus V_{3} \subset V_{2}^{\perp}$. By (a), this inclusion has to be an equality. Therefore, $V_{2}$ is a non-degenerate subspace, and so is $V_{1} \oplus V_{3}$. By (c), $\operatorname{dim} V_{1}, \operatorname{dim} V_{3} \leq \frac{1}{2} \operatorname{dim}\left(V_{1} \oplus V_{3}\right)$. So $\operatorname{dim} V_{1}=\operatorname{dim} V_{3}$. The lemma is proved.

\section{Calculating intertwining: Decompositions}

This section makes heavy use of the groups associated with concave functions by Bruhat-Tits theory. See $\$ 1$ for basic definitions and notation.

Let $\Phi$ be a root system of linear functions on a real vector space $V, \Phi^{0}=\Phi \cup\{0\}$. We first give a few ways to recognize concave functions on $\Phi^{0}$. Recall that our functions take values in $\tilde{\mathbb{R}}$. The proof of the next lemma is very easy. 
Lemma 13.1. In this lemma, "concave" means "concave as a function on $\Phi^{0}$ ".

(i) If $f$ is concave, $v \in V$, then $a \mapsto f(a)+a(v)$ is concave.

(ii) If $f, f^{\prime}$ are concave, so is $a \mapsto \max \left(f(a), f^{\prime}(a)\right)$.

(iii) If $f$ is concave and real-valued and $C \subset \Phi^{0}$ is closed under addition, then

$$
a \mapsto \begin{cases}f(a) & \text { if } a \in C, \\ f(a)+ & \text { if } a \notin C\end{cases}
$$

is also concave.

(iv) If $f$ is concave, and $C \subset \Phi^{0}$ is closed under addition, then

$$
a \mapsto \begin{cases}f(a) & \text { if } a \in C, \\ \infty & \text { if } a \notin C\end{cases}
$$

is also concave.

Let $G$ be a split connected reductive group over $E$, and let $T$ be a maximal $E$-split torus in $G$. Let $\Phi=\Phi(G, T, E)$, and $y$ a point on $A(G, T, E)$. Recall that to a concave function $f$ on $\Phi^{0}$, we can construct a subgroup $G(E)_{y, f}$ of $G(E)$ (the construction actually depends on $T$, but this is suppressed in the notation).

Lemma 13.2. Let $f, f^{\prime}$ be concave. Assume that $f(0)>0, f^{\prime}(0)>0$.

(i) The intersection $G(E)_{y, f} \cap G(E)_{y, f^{\prime}}$ is equal to $G(E)_{y, \max \left(f, f^{\prime}\right) \text {, where }}$ $\max \left(f, f^{\prime}\right)$ is the concave function $a \mapsto \max \left(f(a), f^{\prime}(a)\right)$.

(ii) If the function $\min \left(f, f^{\prime}\right): a \mapsto \min \left(f(a), f^{\prime}(a)\right)$ is concave, then $G(E)_{y, \min \left(f, f^{\prime}\right)}$ is the subgroup generated by $G(E)_{y, f}$ and $G(E)_{y, f^{\prime}}$.

Proof. Choose a system of positive roots $\Phi^{+}$of $\Phi$ and put $\Phi^{-}=\Phi \backslash \Phi^{+}$. It is known that for any fixed order of multiplication, the multiplication map

$$
\prod_{a \in \Phi^{+}} G_{a}(E)_{y, h(a)} \times T(E)_{h(0)} \times \prod_{a \in \Phi^{-}} G_{a}(E)_{y, h(a)} \rightarrow G(E)_{y, h}
$$

is bijective for any concave $h$ such that $h(0)>0$ [BT1 6.4.48]. We fix an order and write $g=\prod_{a \in \Phi^{0}} g_{a}$ to indicate this decomposition for $g \in G(E)_{y, h}$.

Now if $g \in G(E)_{y, f} \cap G(E)_{y, f^{\prime}}$, then $g$ has a decomposition $\prod g_{a}$ as an element of $G(E)_{y, f}$, and also a decomposition $\prod g_{a}^{\prime}$ as an element of $G(E)_{y, f^{\prime}}$. But it is also known that the multiplication map

$$
\prod_{a \in \Phi^{+}} G_{a}(E) \times T(E) \times \prod_{a \in \Phi^{-}} G_{a}(E) \rightarrow G(E)
$$

is injective $[\mathrm{Hu}, 28.1,28.5]$. Therefore the two decompositions must be the same: $g_{a}=g_{a}^{\prime}$ for all $a$. Therefore, $g_{a} \in G_{a}(E)_{y, f(a)} \cap G_{a}(E)_{y, f^{\prime}(a)}=G_{a}(E)_{y, \max \left(f(a), f^{\prime}(a)\right)}$. This shows that $G(E)_{y, f} \cap G(E)_{y, f^{\prime}} \subset G(E)_{y, \max \left(f, f^{\prime}\right)}$. The reverse inclusion is of course trivial. This proves (i).

(ii) is immediate from the definition.

Now assume that $G$ is a connected reductive group over $F$, and $E / F$ is a finite Galois extension such that $G \otimes E$ is split. Let $T$ be a maximal torus of $G$, defined over $F$ split over $E$. Let $y$ be a point in $A(G, T, E) \cap \mathcal{B}(G, F)$.

Under these assumptions, $\operatorname{Gal}(E / F)$ acts on $\Phi=\Phi(G, T, E)$. A function $f$ on $\Phi^{0}$ is called $\operatorname{Gal}(E / F)$-stable if $f(\gamma . a)=f(a)$ for all $a \in \Phi^{0}, \gamma \in \operatorname{Gal}(E / F)$. If $f$ is concave and $\operatorname{Gal}(E / F)$-stable, then $G(E)_{y, f}$ is $\operatorname{Gal}(E / F)$-stable, and we define $G(F)_{y, f}=G(E)_{y, f} \cap G(F)=G(E)_{y, f}^{\operatorname{Gal}(E / F)}$. 
Lemma 13.3. Let $f, f^{\prime}$ be $\operatorname{Gal}(E / F)$-stable concave functions such that $\min \left(f, f^{\prime}\right)$ is also concave, $f(0)>0, f^{\prime}(0)>0$, and $G(E)_{y, f}$ normalizes $G(E)_{y, f^{\prime}}$. Suppose that

$$
H^{1}\left(\operatorname{Gal}(E / F), G(E)_{y, \max \left(f, f^{\prime}\right)}\right)
$$

is trivial. Then $G(F)_{y, f} G(F)_{y, f^{\prime}}=G(F)_{y, \min \left(f, f^{\prime}\right)}$.

Proof. Consider the exact sequence of groups

$$
1 \rightarrow G(E)_{y, \max \left(f, f^{\prime}\right)} \rightarrow G(E)_{y, f} \ltimes G(E)_{y, f^{\prime}} \rightarrow G(E)_{y, \min \left(f, f^{\prime}\right)} \rightarrow 1,
$$

where the first map is $x \mapsto x \ltimes x^{-1}$ and the second map is $x \ltimes y \mapsto x y$. The Galois group $\operatorname{Gal}(E / F)$ acts on all three groups. Taking the exact sequence of cohomology groups, we obtain

$$
\begin{aligned}
1 \rightarrow G(F)_{y, \max \left(f, f^{\prime}\right)} \rightarrow G(F)_{y, f} \ltimes G(F)_{y, f^{\prime}} & \rightarrow G(F)_{y, \min \left(f, f^{\prime}\right)} \\
& \rightarrow H^{1}\left(\operatorname{Gal}(E / F), G(E)_{y, \max \left(f, f^{\prime}\right)}\right) .
\end{aligned}
$$

By assumption, the last term is trivial. Therefore,

$$
G(F)_{y, f} \ltimes G(F)_{y, f^{\prime}} \rightarrow G(F)_{y, \min \left(f, f^{\prime}\right)}
$$

is surjective. This proves the lemma.

In order to obtain the vanishing of $H^{1}$ in Lemma 13.3, we will need a slight generalization of Proposition 2.2

Proposition 13.4. Suppose additionally that $E / F$ is tamely ramified. Let $f$ be a $\operatorname{Gal}(E / F)$-stable concave function on $\Phi^{0}$ such that there is a real number $\epsilon>0$ and $f(a) \geq \epsilon$ for all $a \in \Phi^{0}$. Then

$$
H^{1}\left(\operatorname{Gal}(E / F), G(E)_{y, f}\right)
$$

is trivial.

Proof. By [BT1, 6.4.44], there is a $\operatorname{Gal}(E / F)$-stable concave function $f_{1}$ such that

$$
\left[G(E)_{y, f}, G(E)_{y, f}\right] \subset G(E)_{y, f_{1}}
$$

and $f_{1}(a) \geq 2 \epsilon$ for all $a \in \Phi^{0}$.

Inductively, we can find a sequence of $\operatorname{Gal}(E / F)$-stable concave functions $\left(f_{n}\right)_{n}$ on $\Phi^{0}$ such that $\left[G(E)_{y, f_{n}}, G(E)_{y, f_{n}}\right] \subset G(E)_{y, f_{n+1}}$ and $f_{n}(a) \geq 2^{n} \epsilon$ for all $a \in \Phi^{0}$.

By [BT1, 6.4.48], we have a $\operatorname{Gal}(E / F)$-equivariant isomorphism

$$
G(E)_{y, f_{n}} / G(E)_{y, f_{n+1}} \simeq \mathfrak{g}(E)_{y, f_{n}} / \mathfrak{g}(E)_{y, f_{n+1}} .
$$

The arguments in $\$ 2$ show that these two groups have vanishing first cohomology. The proof is then completed by using Lemma 2.8

We now return to the proof of Proposition 12.3. From now on, $g$ is a fixed element of $G^{\prime}(F)$. Let $T$ be a maximal $F$-torus of $G^{\prime}$ such that $T$ is split over a tamely ramified Galois extension $E$, and $y, g . y \in A\left(G^{\prime}, T, F\right)$. Such a torus exists by the fact that any two points of $\mathcal{B}\left(G^{\prime}, F\right)$ are contained in an apartment of $\mathcal{B}\left(G^{\prime}, F\right)$ and by the discussion in the beginning of $₫ 2$ We put $\Phi=\Phi(G, T, E)$.

Let

$$
\left\{\begin{array}{l}
\Phi_{1}=\{a \in \Phi(G, T, E): a(y-g . y)<0\} \\
\Phi_{2}=\{a \in \Phi(G, T, E): a(y-g . y)=0\} \cup\{0\}, \\
\Phi_{3}=\{a \in \Phi(G, T, E): a(y-g . y)>0\} .
\end{array}\right.
$$


Notice that each $\Phi_{i}$ is closed under addition. Let $\Phi_{i}^{\prime}=\Phi_{i} \cap\left(\Phi\left(G^{\prime}, T, E\right) \cup\{0\}\right)$, $\Phi_{i}^{\prime \prime}=\Phi_{i} \backslash \Phi_{i}^{\prime}, i=1,2,3$. Define $f_{i}: \Phi(G, T, E) \cup\{0\} \rightarrow \tilde{\mathbb{R}}$ as follows (recall that $s=r / 2)$ :

$$
f_{i}(a)= \begin{cases}r & \text { if } a \in \Phi_{i}^{\prime}, \\ s & \text { if } a \in \Phi_{i}^{\prime \prime}, \\ \infty & \text { if } a \notin \Phi_{i} .\end{cases}
$$

Lemma 13.1(iv) shows that $f_{1}, f_{2}, f_{3}$ are concave functions, because $f_{0}=f_{(r, s)}$ is.

We define $J_{i}(E)$ to be the subgroup $G(E)_{y, f_{i}}$ of $G(E)$ for $i=0,1,2,3$. We have $J_{0}(E)=J(E)=J_{1}(E) J_{2}(E) J_{3}(E)$ by [BT1, 6.4.48]. Notice also that $\Phi_{1} \cup \Phi_{2}$ and $\Phi_{2} \cup \Phi_{3}$ are closed under addition. Therefore, $J_{1}(E) J_{2}(E)$ and $J_{2}(E) J_{3}(E)$ are groups. Let $f_{+}=f_{(r, s+)}$ so that $G(E)_{y, f_{+}}=J_{+}(E)$.

Lemma 13.5. All of the following commutator subgroups are contained in $N(E)=$ $\operatorname{ker}\left(\phi_{E}: J_{+}(E) \rightarrow \mathbb{C}^{*}\right)$ :

$$
\left[J_{1}(E), J_{1}(E)\right],\left[J_{1}(E), J_{2}(E)\right],\left[J_{2}(E), J_{3}(E)\right],\left[J_{3}(E), J_{3}(E)\right] .
$$

Proof. First we note that these commutators are all contained in $J_{+}(E)$, and an element $g$ of $J_{+}(E)$ has a decomposition $\prod_{a \in \Phi^{0}} g_{a}$, where $g_{a} \in G_{a}(F)_{y, f_{+}(a)}$. By the construction of $\hat{\phi}, \hat{\phi}(g) \neq 1$ only when $g_{0} \notin G_{0}(E)_{r+}$.

We can apply [BT1, 6.4.44] to construct a concave function $h$ such that $\left[J_{1}(E)\right.$, $\left.J_{2}(E)\right] \subset G(E)_{y, h}$. By the preceding discussion, it suffices to show that $h(0)>r$. It follows from [BT1, 6.4.44] that $h(a) \neq \infty$ only when $a$ can be expressed as a sum $\sum_{i} a_{i}+\sum b_{j}$, where $\left(a_{i}\right)_{i} \subset \Phi_{1}$ and $\left(b_{j}\right)_{j} \subset \Phi_{2}$ are non-empty families. Clearly this condition implies that $a(y-g \cdot y)<0$. Therefore, $h(0)=\infty$ and $\hat{\phi}\left(\left[J_{1}(E), J_{2}(E)\right]\right)=1$.

The remaining three cases are proved similarly.

Define

$$
\left\{\begin{array}{l}
f_{g}(a)=f_{0}(a)+a(y-g \cdot y) \\
f_{g+}(a)=f_{+}(a)+a(y-g \cdot y) \\
f_{4}(a)=\max \left(f_{0}(a), f_{g}(a)\right) \\
f_{5}(a)=\max \left(f_{0}(a), f_{g+}(a)\right) \\
f_{6}(a)=\min \left(f_{4}(a), f_{+}(a)\right) \\
f_{7}(a)=\min \left(f_{5}(a), f_{+}(a)\right) \\
f_{8}(a)=\min \left(f_{1}(a), f_{2}(a)\right)
\end{array}\right.
$$

Lemma 13.1 shows that these functions are concave on $\Phi^{0}$ (the roman numeral following each function indicates the case of Lemma [13.1 to use).

Recall that for any $a \in \Phi(G, T, E) \cup\{0\}$, we have $G_{a}(E)_{g . y, r}=G_{a}(E)_{y, r+a(y-g . y)}$. It follows easily that $G(E)_{y, f_{g}}={ }^{g} J(E), G(E)_{y, f_{g+}}={ }^{g} J_{+}(E), G(E)_{y, f_{4}}=g \cap J(E)$, $G(E)_{y, f_{5}}=J(E) \cap\left({ }^{g} J_{+}(E)\right), G(E)_{y, f_{6}}=J_{1}(E) J_{2}(E) J_{+}(E)=\left({ }^{g \cap} J(E)\right) J_{+}(E)$, $G(E)_{y, f_{7}}=J_{1}(E) J_{+}(E)=\left(J(E) \cap\left({ }^{g} J_{+}(E)\right)\right) J_{+}(E)$, and $G(E)_{y, f_{8}}=J_{1}(E) J_{2}(E)$.

We will now descend to $F$. Notice that each of $\Phi_{i}, \Phi_{i}^{\prime}, \Phi_{i}^{\prime \prime}$ is Gal $(E / F)$-stable, and therefore $f_{i}(0 \leq i \leq 8), f_{+}, f_{g}, f_{g+}$ are all $\mathrm{Gal}(E / F)$-stable. Therefore, $J_{i}(E)$ is $\operatorname{Gal}(E / F)$-stable. We define $J_{i}=J_{i}(E) \cap G(F), i=1,2,3$.

Lemma 13.6. The four conditions (a)-(d) in Lemma12.8 are all satisfied. 
Proof. (b) and (c) are obvious from the preceding lemma. If $i, j, k$ are distinct, then $J_{i} J_{+} \cap J_{j} J_{k} J_{+} \subset J_{i}(E) J_{+}(E) \cap J_{j}(E) J_{k}(E) J_{+}(E) \subset J_{+}(E)$ by Lemma 13.2 Therefore, $J_{i} J_{+} \cap J_{j} J_{k} J_{+} \subset J_{+}(E) \cap G(F)=J_{+}$. Therefore, we know that the sum $V_{1}+V_{2}+V_{3}$ is direct.

It remains to show that $J_{1} J_{2} J_{3}=J, J_{1} J_{+}=\left(J \cap\left({ }^{g} J_{+}\right)\right) J_{+}$, and $J_{1} J_{2} J_{+}=$ $(g \cap J) J_{+}$.

It is easy to show that $J_{1} J_{2}=G(F)_{y, f_{8}}$ and $J_{1} J_{2} J_{3}=J$.

We now claim that $J_{1} J_{+}=G(F)_{y, f_{7}}=\left(J \cap\left({ }^{g} J_{+}\right)\right) J_{+}$. By Lemma 13.3, the two equalities are implied by

$$
H^{1}\left(\operatorname{Gal}(E / F), G(E)_{y, \max \left(f_{1}, f_{+}\right)}\right)=1 \quad \text { and } \quad H^{1}\left(\operatorname{Gal}(E / F), G(E)_{y, \max \left(f_{5}, f_{+}\right)}\right)=1 .
$$

The vanishing of these first cohomology groups, as well as those needed below, can be checked easily by Proposition 13.4 .

We claim that $G(F)_{y, f_{8}} J_{+}=G(F)_{y, f_{6}}=G(F)_{y, f_{4}} J_{+}$. This is implied by

$H^{1}\left(\operatorname{Gal}(E / F), G(E)_{y, \max \left(f_{8}, f_{+}\right)}\right)=1 \quad$ and $\quad H^{1}\left(\operatorname{Gal}(E / F), G(E)_{y, \max \left(f_{4}, f_{+}\right)}\right)=1$.

The lemma is proved completely.

Proof of Proposition 12.3. Combine the above lemma with Lemmas 12.6 and 12.8

We want to derive one more decomposition needed in the next section. Recall that $K=G^{\prime}(F) \cap G(F)_{[y]}$.

Lemma 13.7. For all $g \in G^{\prime}(F),{ }^{g \cap}(K J)=\left({ }^{g \cap} K\right)\left({ }^{g \cap} J\right)$.

Proof. Again we will prove the lemma first over $E$. We do all the constructions over $E$ to define $K(E), J(E)$, etc.

Choose an ordering of $\Phi=\Phi(G, T, E)$. We define $U(+)$ (resp. $U(-))$ to be the subgroup generated by $G_{a}(E)$ for all positive (resp. negative) $a \in \Phi(G, T, E)$ such that $a \notin \Phi\left(G^{\prime}, T, E\right)$. Then the multiplication map $G^{\prime}(E) \times U(+) \times U(-) \rightarrow G(E)$ is injective.

We also define $J(+)$ (resp. $J(-))$ to be the subgroup generated by $G_{a}(E)_{y, r / 2}$ for all positive (resp. negative) $a \in \Phi(G, T, E)$ such that $a \notin \Phi\left(G^{\prime}, T, E\right)$.

The group $K(E) J(E)$ is generated by $K(E), J(+)$, and $J(-)$. We claim that the multiplication map $K(E) \times J(+) \times J(-) \rightarrow K(E) J(E)$ is a bijection. Indeed, we have $K(E) J(+) J(-)=K(E) G^{\prime}(E)_{y, r} J(+) J(-)=K(E) J(E)$. So the map is surjective. The injectivity is obvious from the above discussion.

It is clear that $G^{\prime}(E)$ normalizes $G^{\prime}(E), U(+)$ and $U(-)$. Therefore, if $x \in$ $g \cap(K(E) J(E))$ is decomposed into $x_{1} x_{2} x_{3}$ as an element of $K(E) J(+) J(-)$, and decomposed into $x_{1}^{\prime} x_{2}^{\prime} x_{3}^{\prime}$ as an element of ${ }^{g} K(E)^{g} J(+)^{g} J(-)$, we must have $x_{1}=$ $x_{1}^{\prime} \in{ }^{g \cap} K(E), x_{2}=x_{2}^{\prime} \in{ }^{g \cap} J(+), x_{3}=x_{3}^{\prime} \in{ }^{g \cap} J(-)$. Therefore, we have $g \cap(K(E) J(E))=\left({ }^{g \cap} K(E)\right)(g \cap J(E))$.

As in the proof of Lemma[13.3 it remains to show that

$$
H^{1}(\operatorname{Gal}(E / F), K(E) \cap J(E))=1 \quad \text { and } \quad H^{1}\left(\operatorname{Gal}(E / F),{ }^{g \cap}(K(E) \cap J(E))\right)=1 .
$$

The first statement is a special case of Proposition 2.2 because $K(E) \cap J(E)=$ $G^{\prime}(E)_{y, r}$. The second statement can be handled by Proposition 13.4 


\section{Calculating intertwining: Conclusion}

So far, we have only assumed that $\phi$ is a $G$-generic-character of $G(F)_{y, r: r+\text {. }}$. Now we assume further that $\phi$ is a quasi-character defined on the whole of $G^{\prime}(F)$, $G$-generic relative to $y$, of depth $r$.

As in 4 , we inflate $\left.\phi\right|_{K}$ to a representation $\inf (\phi)$ of $K \ltimes J$. It can be shown as in $₫$ that the representation $\inf (\phi) \otimes \tilde{\phi}$ of $K \ltimes J$ is inflated from a representation $\phi^{\prime}$ of $K J$ via the map $K \ltimes J \rightarrow K J$.

Proposition 14.1. For all $g \in G^{\prime}(F)$, we have $\operatorname{dim} I_{g}\left(\left.\phi^{\prime}\right|_{K J}\right)=1$.

The proof will take up the whole section. We first record a consequence.

Theorem 14.2. For any subgroup $K^{\prime}$ such that $J \subset K^{\prime} \subset K J$, and for all $g \in$ $G^{\prime}(F)$, we have $\operatorname{dim} I_{g}\left(\left.\phi^{\prime}\right|_{K^{\prime}}\right)=1$.

Proof. Immediate from Proposition 12.3 and Proposition 14.1.

We now begin to prove Proposition 14.1. From Lemma 13.7 $\operatorname{Hom}_{g \cap(K J)}\left({ }^{g} \phi^{\prime}, \phi^{\prime}\right)$ is the same as

$$
\operatorname{Hom}_{g \cap K \ltimes g \cap J}\left({ }^{g} \inf (\phi) \otimes{ }^{g} \tilde{\phi}, \inf (\phi) \otimes \tilde{\phi}\right) .
$$

Clearly, ${ }^{g} \inf (\phi)=\inf (\phi)$ on ${ }^{g} K \ltimes{ }^{g} J$ ( $\phi$ is a 1-dimensional character on the whole of $\left.G^{\prime}(F)\right)$. Therefore, it suffices to show that $\operatorname{dim} \operatorname{Hom}_{g \cap K \ltimes g \cap J}\left({ }^{g} \tilde{\phi}, \tilde{\phi}\right)=1$. From Proposition 12.3, we know that this dimension is at most one.

The rest of the proof refines the discussion in Remark [12.7 We adopt the notation there: $U=\left({ }^{g \cap} J\right) J_{+} / J_{+}, U_{0}=\left(J \cap\left({ }^{g} J_{+}\right)\right) J_{+} / J_{+}$. Furthermore, let $U^{\#}$ and $U_{0}^{\#}$ be the preimage of $U$ and $U_{0}$ by the projection $V^{\#} \rightarrow V$.

The character $\left.\hat{\phi}\right|_{J_{+}}$agrees with the character $\left.{ }^{g} \hat{\phi}\right|_{\left(J \cap\left({ }^{g} J_{+}\right)\right)}$on the intersection of their domains of definition by Lemma 9.3 Together they determine a character of $\left(J \cap\left(J_{+}\right)\right) J_{+}$which is trivial on $N$. We denote this character by $\phi_{g}$. Our first task is to identify this character. Recall that $j: J / N \rightarrow\left(J / J_{+}\right)^{\sharp}$ is the special isomorphism defined in Proposition 11.4 .

Lemma 14.3. The character $\phi_{g}$ is trivial on $j^{-1}\left(U_{0} \times\{1\}\right)$, hence $\operatorname{ker} \phi_{g}$ is equal to $j^{-1}\left(U_{0} \times\{1\}\right)$.

Proof. The second statement follows from the first because

$$
\left(J \cap\left({ }^{g} J_{+}\right)\right) J_{+} / j^{-1}\left(U_{0} \times\{1\}\right) \simeq U_{0}^{\#} /\left(U_{0} \times\{1\}\right)
$$

is cyclic of order $p$ and $\phi_{g}$ is non-trivial.

We first prove the lemma for the split case. According to the proof of Proposition 11.4, $U_{0} \times\{1\}$ is generated by $j(u), u \in G_{a}(F)_{y, r / 2}, a \in \Phi_{1}^{\prime \prime}$, where $\Phi_{1}^{\prime \prime}$ is defined in the last section. It is clear that ${ }^{g} \hat{\phi}(u)=1$. This implies the lemma in the split case.

The general case is easily reduced to the split case by looking at the proof of Proposition 11.4.

Since ${ }^{g \cap} J$ is contained in $J$, the pairing $\langle\rangle:,(a, b) \mapsto \hat{\phi}\left(a b a^{-1} b^{-1}\right)$ is defined on $g \cap J$. The character $\phi$ is also $G$-generic relative to $g . y$, and the associated " $J$ "group is $\left(G^{\prime}, G\right)(E)_{g . y,(r, s)}={ }^{g} J$. The pairing ${ }^{g}\langle$,$\rangle on { }^{g} J$ is related to $\langle$,$\rangle by$ ${ }^{g}\left\langle g a g^{-1}, g b g^{-1}\right\rangle=\langle a, b\rangle$. However, for $a, b \in{ }^{g \cap} J$, we have $\langle a, b\rangle={ }^{g}\langle a, b\rangle$ by Lemma 9.3 
Let $\left({ }^{g \cap} J\right)_{+}=\left\{a \in{ }^{g \cap} J:\left\langle a,{ }^{g \cap} J\right\rangle=1\right\}$.

Lemma 14.4. $\left({ }^{g \cap} J\right)_{+}={ }^{g \cap} J \cap\left(\left({ }^{g} J_{+} \cap J\right) J_{+}\right)=\left({ }^{g} J \cap J_{+}\right)\left({ }^{g} J_{+} \cap J\right)$.

Proof. By Remark 12.7

$$
\frac{g \cap J}{(g \cap J)_{+}} \simeq \frac{(g \cap J) J_{+}}{\left(g J_{+} \cap J\right) J_{+}} \simeq \frac{g \cap J}{g \cap J \cap\left(\left({ }^{g} J_{+} \cap J\right) J_{+}\right)}
$$

(the second equality is from a standard isomorphism theorem). This proves the first equality in the lemma.

The second equality of the lemma can be verified by the method of the last section: first deal with the split case and write every group as a group associated to a concave function and apply Lemma 13.2 then reduce the general case to the split case.

We put $N_{g}=\operatorname{ker}\left(\left.\phi_{g}\right|_{(g \cap J)_{+}}\right), N_{g}^{\prime}=\operatorname{ker}\left(\phi_{g}\right)$, and summarize the situation with the following diagram:

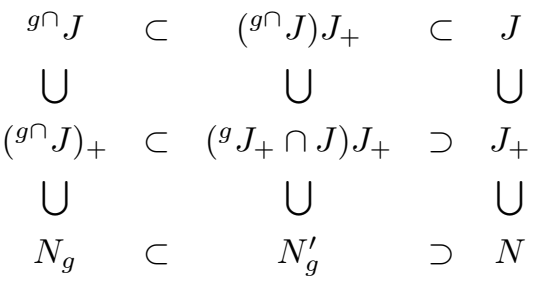

We obtain

$$
\frac{g \cap J}{N_{g}} \hookrightarrow \frac{(g \cap J) J_{+}}{N_{g}^{\prime}} \hookrightarrow \frac{J}{N_{g}^{\prime}} \stackrel{j}{\simeq} \frac{V^{\sharp}}{j\left(N_{g}^{\prime}\right)} .
$$

By Lemma 14.3 the image of the leftmost term in the rightmost term is $U^{\sharp} /$ $\left(U_{0} \times\{1\}\right)$.

Lemma 14.5. The action of ${ }^{g \cap} K$ on $J / J_{+}$stabilizes the subspace $U_{0}$.

Proof. This is obvious: $\left[{ }^{g} K \cap K,{ }^{g} J_{+} \cap J\right] \subset{ }^{g} J_{+} \cap J$.

We now digress to discuss a general property of Weil representations. Let $(V,\langle\rangle, C$,$) be a non-degenerate symplectic space over \mathbb{F}_{p}$, and let $(\omega, W)$ be the Weil representation of $\operatorname{Sp}(V) \ltimes V^{\sharp}$. Let $U_{0}$ be a totally isotropic subspace of $V$ and $U=U_{0}^{\perp}$. For any subspace $X$ of $V$, let $X^{\sharp}$ be the preimage of $X$ under $V^{\sharp} \rightarrow V$, i.e. $X^{\sharp}=X \times C$, and let $X^{0}$ be $X \times\{1\}$. Let $P_{0}=\left\{g \in \operatorname{Sp}(V): g \cdot U_{0} \subset U_{0}\right\}$ (this is a maximal parabolic subgroup of $\operatorname{Sp}(V))$. There is a surjection $P_{0} \rightarrow \operatorname{Sp}\left(U / U_{0}\right)$. Let $N_{0}$ be its kernel.

Lemma 14.6. The group $U^{\sharp} / U_{0}^{0}$ is naturally isomorphic to $\left(U / U_{0}\right)^{\sharp}$ by the map $(u, c)+U_{0}^{0} \mapsto\left(u+U_{0}, c\right)$. The subspace $W^{U_{0}^{0}}$ is stable under the action of $P_{0} \ltimes U^{\sharp}$ and the action of $N_{0} \ltimes U_{0}^{0}$ is trivial. Therefore, we can regard $W^{U_{0}^{0}}$ as a representation of $\operatorname{Sp}\left(U / U_{0}\right) \ltimes\left(U / U_{0}\right)^{\sharp}$. This representation is simply the Weil representation of $\operatorname{Sp}\left(U / U_{0}\right) \ltimes\left(U / U_{0}\right)^{\sharp}$.

Proof. The first claim, i.e. that $U^{\sharp} / U_{0}^{0}$ is naturally isomorphic to $\left(U / U_{0}\right)^{\sharp}$, is obvious.

It is also obvious that $W^{U_{0}^{0}}$ is stable under the action of $P_{0} \ltimes U^{\sharp}$, and $U_{0}^{0}$ acts trivially on this subspace. To show that $N_{0}$ acts trivially on $W^{U_{0}^{0}}$, we first notice 
that $W^{U_{0}^{0}}$ as a representation of $\left(U / U_{0}\right)^{\sharp}$ is simply the Heisenberg representation (Lemma 12.2), and therefore is irreducible. There is at most one way of extending this representation to a representation of $P_{0} \ltimes\left(U / U_{0}\right)^{\sharp}$ up to tensoring by a linear character of $P_{0}$. Therefore, $W^{U_{0}^{0}}$ as a representation of $P_{0} \ltimes\left(U / U_{0}\right)^{\sharp}$ is the (inflation of the) Weil representation tensored by a linear character of $P_{0}$. It is easy to show that $N_{0}$ is contained in the commutator subgroup of $P_{0}$. Therefore, $N_{0}$ acts trivially on $W^{U_{0}^{0}}$.

Let $V_{1}$ be a non-degenerate subspace of $V$ such that $U=U_{0} \oplus V_{1}$. Put $V_{2}=V_{1}^{\perp}$. It is well known (see $\mathrm{Ge}$ ) that the pull back of $\omega$ by the map

$$
\left(\operatorname{Sp}\left(V_{1}\right) \ltimes V_{1}^{\sharp}\right) \times\left(\operatorname{Sp}\left(V_{2}\right) \ltimes V_{2}^{\sharp}\right) \rightarrow \operatorname{Sp}(V) \ltimes V^{\sharp}
$$

is the external tensor product of the Weil representation $\omega_{1}$ of $\operatorname{Sp}\left(V_{1}\right) \ltimes V_{1}^{\sharp}$ with the Weil representation $\omega_{2}$ of $\operatorname{Sp}\left(V_{2}\right) \ltimes V_{2}^{\sharp}$. From this it follows that $W^{U_{0}^{0}}$ is the Weil representation of $\operatorname{Sp}\left(V_{1}\right) \ltimes V_{1}^{\sharp}$ (we identify $\operatorname{Sp}\left(V_{1}\right)$ with the first factor of $\operatorname{Sp}\left(V_{1}\right) \times$ $\left.\operatorname{Sp}\left(V_{2}\right) \subset \operatorname{Sp}(V)\right)$. Since $\operatorname{Sp}\left(V_{1}\right) \ltimes V_{1}^{\sharp}$ maps isomorphically onto $P_{0} / N_{0} \ltimes U^{\sharp} / U_{0}^{0}$, the space $W^{U_{0}^{0}}$ as a representation of $\operatorname{Sp}\left(U / U_{0}\right) \ltimes\left(U / U_{0}\right)^{\sharp}$ is the Weil representation. This proves the last statement of the lemma.

We now return to the main proof. Let $j_{1}$ be the composition

$$
\frac{g \cap J}{N_{g}} \simeq \frac{U^{\#}}{U_{0} \times\{1\}} \simeq\left(\frac{U}{U_{0}}\right)^{\#},
$$

where the first isomorphism is the one in the discussion preceding Lemma 14.5, and the second isomorphism is from the first statement of Lemma 14.6.

Proposition 14.7. (i) The group ${ }^{g \cap} J / N_{g}$ is a Heisenberg p-group with center $\left({ }^{g \cap} J\right)_{+} / N_{g}$, and $j_{1}:\left({ }^{g \cap} J\right) / N \rightarrow\left({ }^{g \cap} J /\left({ }^{g \cap} J\right)_{+}\right)^{\sharp}$ is a special isomorphism. (ii) Let $f_{1}:{ }^{g \cap} K \rightarrow \operatorname{Sp}\left(g \cap J / N_{g}\right)$ be the natural morphism induced by conjugation. Then $\left(f_{1}, j_{1}\right)$ is a symplectic action. (iii) Let $\tilde{\phi}$ be the Weil representation of $K \ltimes J$, and let $\pi_{1}$ be the $\left(\left.\phi_{g}\right|_{g} J_{+} \cap J\right)$-isotypic subspace of $\tilde{\phi}$. Then $\pi_{1}$ is naturally a representation of ${ }^{g \cap} K \ltimes^{g \cap} J$, and this representation is the Weil representation coming from the symplectic action $\left(f_{1}, j_{1}\right)$.

Proof. (i) is clear.

Let $k \in K_{1}$. Since $(f, j)$ is a symplectic action, there exists $\bar{f}(k) \in \operatorname{Sp}(V)$ such that $j \circ f(k) \circ j^{-1}(u, c)=(\bar{f}(k) . u, c)$. It follows that $j_{1} \circ f_{1}(k) \circ j_{1}^{-1}\left(u+U_{0}, c\right)=$ $\left(\bar{f}(k) \cdot u+U_{0}, c\right)$. By Lemma 14.5, $\bar{f}(k)$ stabilizes $U_{0}$ and $U=U_{0}^{\perp}$. Therefore, $\bar{f}(k)$ induces a symplectic automorphism on $U / U_{0}$. This shows that $\left(f_{1}, j_{1}\right)$ is a symplectic action and proves (ii).

By Lemma 14.3 the $\left(\left.\phi^{*}\right|_{g} \cap J_{+}\right)$-isotypic subspace of $\tilde{\phi}$ is the same as the subspace fixed by $j^{-1}\left(U_{0} \times\{1\}\right)$. Therefore, (iii) follows from the last statement of Lemma 14.6 .

Proof of Proposition 14.1. We can exchange the roles of $J$ and ${ }^{g} J$ in the discussion above. More precisely, we can replace $y$ by $y^{\bullet}=g . y$ and $g$ by $g^{\bullet}=g^{-1}$, and then apply the above discussion. This gives us another special isomorphism $j_{1}^{\bullet}$ : $\left({ }^{g \cap} J\right) / N_{g} \rightarrow\left({ }^{g \cap} J /\left({ }^{g \cap} J\right)_{+}\right)^{\sharp}$, a morphism $f_{1}^{\bullet}:{ }^{g \cap} K \rightarrow \operatorname{Sp}\left({ }^{g \cap} J / N_{g}\right)$, and a subspace $\pi_{1}^{\bullet}$ of ${ }^{g} \tilde{\phi}$ which is $\left(\left.\phi_{g} \bullet\right|_{g} J_{J_{+}}\right)$-isotypic and naturally the Weil representation of $g \cap K \ltimes{ }^{g \cap} J$ coming from the symplectic action $\left(f_{1}^{\bullet}, j_{1}^{\bullet}\right)$. 
Obviously, $f_{1}=f_{1}^{\bullet}$. By tracing the proofs of Proposition 11.4 and Proposition 14.7, we see that $j_{1}=j_{1}^{\bullet}$. Therefore, we have found a subspace $\pi_{1}$ of $\tilde{\phi}$ and a subspace $\pi_{1}^{\bullet}$ of ${ }^{g} \tilde{\phi}$ which are isomorphic as representations of ${ }^{g \cap} K \ltimes^{g \cap} J$. This completes the proof of Proposition 14.1

\section{Supercuspidal REPRESENTATIONS AND SUPERCUSPIDAL TYPES}

We now resume the situation of $\$ 3$ We say that the datum $(\vec{G}, y, \vec{r}, \rho, \vec{\phi})$ is generic if $\phi_{i}$ is $G^{i+1}$-generic of depth $r_{i}$ (relative to $y$ ) for $0 \leq i \leq d-1$.

Now suppose that the datum $(\vec{G}, y, \vec{r}, \rho, \vec{\phi})$ is generic. By Theorem 9.4, condition $\mathbf{S C 1}_{i}$ is satisfied. By Theorem 11.5, condition $\mathbf{S C 2}{ }_{i}$ is satisfied with canonically constructed $\tilde{\phi}_{i}$ 's, which do not depend on any choices. Finally, condition $\mathbf{S C 3}_{i}$ is also satisfied by Theorem 14.2, Applying Proposition 4.6, we obtain the following theorem.

Theorem 15.1. Let $(\vec{G}, y, \vec{r}, \rho, \vec{\phi})$ be a generic datum. Construct $\left(\rho_{i}\right)_{i}$ by the method of $\$ 4$ using $\left(\tilde{\phi}_{i}\right)_{i}$ constructed in $\$ 11$. Then the compactly induced representation $\pi_{i}=\operatorname{ind}_{K^{i}}^{G^{i}(F)} \rho_{i}$ of $G^{i}(F)$ is irreducible, supercuspidal, and of depth $r_{i}$ for $0 \leq i \leq d$.

We emphasize that the entire construction depends only on the generic datum. No choices are involved.

Remark 15.2. If $G^{0}$ is anisotropic modulo $\mathcal{Z}(G)$, the theorem follows directly from Corollary 4.5. If $J^{i}=J_{+}^{i}$ for $1 \leq i \leq d$, the proof can also be considerably simplified. These cases are known to J. Adler independently.

Corollary 15.3. Let $^{\circ} K^{0}=G^{0}(F)_{y},{ }^{\circ} K^{i}=\left({ }^{\circ} K^{0}\right) \vec{G}^{(i)}(F)_{y,\left(0, s_{0}, \ldots, s_{i-1}\right)}$, and ${ }^{\circ} \rho_{i}=$ $\left.\rho_{i}\right|_{{ }^{\circ} K^{i}}$. Then $\left({ }^{\circ} K^{i},{ }^{\circ} \rho_{i}\right)$ is an $\mathfrak{s}_{i}$-type in the sense of Bushnell-Kutzko ([BK2]), where $\mathfrak{s}_{i}$ is the inertial class of $\left[G^{i}, \pi_{i}\right]_{G^{i}}$.

Proof. This is immediate from [BK2, 5.4], and the fact that ${ }^{\circ} K^{i}$ is the maximal compact subgroup of $K^{i}$.

Remark 15.4. If we only want to construct the supercuspidal type $\left({ }^{\circ} K^{i},{ }^{\circ} \rho_{i}\right)$, we can proceed as follows. Start with a datum $\left(\vec{G}, y, \vec{r},{ }^{\circ} \rho, \vec{\phi}\right)$, satisfying D1, D2, D3, D5, and ${ }^{\circ} \mathbf{D} 4$ :

${ }^{\circ} \mathbf{D} 4{ }^{\circ} \rho$ is an irreducible representation of $G^{0}(F)_{y}$ such that $\left.{ }^{\circ} \rho\right|_{G(F) y, 0+}$ is $\mathbf{1 -}$ isotypic, $G^{0}(F)_{y, 0}$ is a maximal parahoric subgroup, ${ }^{\circ} \rho$ can be extended to a representation of $K^{0}=G^{0}(F)_{[y]}$, and $\left.{ }^{\circ} \rho\right|_{G^{0}(F)_{y, 0: 0+}}$ contains an irreducible cuspidal representation.

We then follow the construction in 4 , and replace $K^{i}$ by ${ }^{\circ} K^{i}, \rho$ by ${ }^{\circ} \rho$, and $\rho_{i}$ by ${ }^{\circ} \rho^{i}$ throughout.

Corollary 15.5. Assume the notation of the preceding corollary. If $g \in G^{i}(F)$ intertwines ${ }^{\circ} \rho_{i}$, then $g \in\left({ }^{\circ} K^{i}\right) G^{0}(F)\left({ }^{\circ} K^{i}\right)$. Moreover, an element $g$ of $G^{0}(F)$ intertwines ${ }^{\circ} \rho_{i}$ if and only if it intertwines ${ }^{\circ} \rho_{0}$. In fact, we have for $g \in G^{0}(F)$,

$$
I_{g}\left(\left.\rho_{i}\right|_{\circ K^{i}}\right)=I_{g}\left(\left.\rho_{0}\right|_{\circ} K^{0}\right) \otimes I_{g}\left(\tilde{\phi}_{0}\right) \otimes \cdots \otimes I_{g}\left(\tilde{\phi}_{i-1}\right),
$$

where $I_{g}\left(\tilde{\phi}_{j}\right)$ is 1-dimensional for $0 \leq j \leq d-1$. 
Proof. This follows by inspecting the proof of Proposition 4.6. At the end of that proof, we will have an equality $I_{g}\left(\inf \left({ }^{\circ} \rho_{i-1}^{\prime}\right)\right)=I_{g}\left({ }^{\circ} \rho_{i-1}^{\prime}\right)$ instead of just an inclusion. This follows from a slight variation of Lemma 13.7

$$
{ }^{g}\left({ }^{\circ} K^{i}\right)=\left({ }^{g}\left({ }^{\circ} K^{i-1}\right)\right)\left({ }^{g} J^{i}\right) .
$$

Each $I_{g}\left(\tilde{\phi}_{j}\right)$ is 1-dimensional by Proposition 12.3

Denote by $\pi(\rho)=\pi(\vec{G}, y, \vec{r}, \rho, \vec{\phi})$ the representation $\operatorname{ind}_{K^{d}}^{G(F)} \rho_{d}$ constructed in Theorem 15.1 We want to show that $\pi(\rho)$ is equivalent to $\pi\left(\rho^{\bullet}\right)$ if $\rho^{\bullet}$ is another representation of $K^{0} / K_{+}^{0}$ such that $\operatorname{ind}_{K^{0}}^{G^{0}(F)} \rho^{\bullet}=\pi_{0}$. By examining the construction, we see that there is a representation $\lambda$ of $K^{d}$ such that $\rho_{d}=\rho \otimes \lambda$, and $\rho_{d}^{\bullet}=\rho^{\bullet} \otimes \lambda$, where we are regarding $\rho$ and $\rho^{\bullet}$ as representations of $K^{d}$ by the natural isomorphism $K^{d} / K_{+}^{d} \simeq K^{0} / K_{0}^{+}$.

Lemma 15.6. If $g \in G^{0}(F)$ is such that

$$
\operatorname{Hom}_{g \cap} K^{0}\left({ }^{g} \rho, \rho^{\bullet}\right) \neq 0,
$$

then

$$
\operatorname{Hom}_{g \cap K^{d}}\left({ }^{g}(\rho \otimes \lambda), \rho^{\bullet} \otimes \lambda\right) \neq 0 .
$$

Proof. By (the proof of) the preceding corollary,

$$
\operatorname{dim} \operatorname{Hom}_{g \cap} K^{d}\left({ }^{g} \lambda, \lambda\right)=1
$$

for all $g \in G^{0}(F)$. The lemma follows immediately.

This lemma implies immediately that $\pi(\rho) \simeq \pi\left(\rho^{\bullet}\right)$. Therefore,

Theorem 15.7. For $0 \leq i \leq d$, the equivalence class of the representation $\pi$ constructed in Theorem 15.1 depends only on the triple $\left(\vec{G}, \pi_{0}, \vec{\phi}\right)$ determined by the 5-tuple $(\vec{G}, y, \vec{r}, \rho, \vec{\phi})$ via Remark 3.7.

In our definition of a twisted Levi sequence, successive members in the sequence are always distinct. Sometimes it is convenient to relax this condition. We call a sequence $\vec{G}=\left(G^{i}\right)_{0 \leq i \leq d}$ of connected reductive groups over $F$ a generalized twisted Levi sequence if $G^{0} \subset G^{1} \subset \cdots \subset G^{d}$ and $G^{i} \otimes \bar{F}$ is a Levi subgroup of $G^{j} \otimes \bar{F}$ for all $i \leq j$. A generalized twisted Levi sequence becomes a twisted Levi sequence if we remove the repeated members.

For example, $\left(G^{\prime}, G\right)$ is a generalized twisted Levi sequence if $G^{\prime}=G$. In this case, we regard any element of $\left(\operatorname{Lie}\left(Z^{\prime}\right)^{\circ}\right)_{-r}$ as generic of depth $r$, and we regard any character trivial on $G^{\prime}(F)_{y, r+}$ as $G$-generic of depth $r$ (in particular, the trivial character is regarded as $G$-generic of depth $r$ for all $r$ ). It is easy to see that Theorem 9.4 Theorem 11.5, and Theorem 14.2 remain true if we allow $\left(G^{\prime}, G\right)$ to be a generalized twisted Levi sequence.

We define a generalized datum to be a 5 -tuple $(\vec{G}, y, \vec{r}, \rho, \vec{\phi})$ satisfying D1-D5, except in $\mathbf{D} 1$ we only require $\vec{G}$ to be a tamely ramified generalized twisted Levi sequence, and in D5 we do not insist that $\phi_{i}$ is non-trivial on $G^{i}(F)_{y, r_{i}}$ (in particular, $\phi_{i}=\mathbf{1}$ is allowed).

Starting with a generalized datum $(\vec{G}, y, \vec{r}, \rho, \vec{\phi})$, it is easy to see that the whole construction of $\$ 4$ can be carried out. Moreover, if the datum is generic (which means that $\phi_{i}$ is $G^{i+1}$-generic of depth $r_{i}$ for $\left.0 \leq i \leq d-1\right)$, then $\pi_{i}=\operatorname{ind}_{K^{i}}^{G^{i}(F)} \rho_{i}$ is irreducible supercuspidal of depth $\leq r_{i}$ for $0 \leq i \leq d$. 
However, it is easy to see that we don't get any new supercuspidal representations using generic generalized data: what we get are exactly those which come from generic data. The point of this discussion is the following proposition, whose proof is easy.

Proposition 15.8. Let $G_{1}, \ldots, G_{n}$ be connected reductive groups, and let $G$ be the direct product $\prod_{j=1}^{n} G_{j}$.

(i) A generalized twisted Levi sequence $\vec{G}$ in $G$ is of the form $\left(\prod_{j} G_{j}^{i}\right)_{0 \leq i \leq d}$, where $\vec{G}_{j}=\left(G_{j}^{i}\right)_{0 \leq i \leq d}$ is a generalized twisted Levi sequence in $G_{j}$ for $1 \leq j \leq n$.

(ii) Let $(\vec{G}, y, \vec{r}, \rho, \vec{\phi})$ be a generalized datum. Then we can write $y=\left(y_{j}\right)_{1 \leq j \leq n}$, $\rho=\bigotimes_{j=1}^{n} \rho^{(j)}, \phi_{i}=\bigotimes_{j=1}^{n} \phi_{i}^{(j)}$, and $\left(\vec{G}_{j}, y_{j}, \vec{r}, \rho^{(j)}, \vec{\phi}^{(j)}\right)$ is a generalized $d a-$ tum for each $j$. Conversely, given a sequence $\left\{\left(\vec{G}_{j}, y_{j}, \vec{r}, \rho^{(j)}, \vec{\phi}^{(j)}\right)\right\}$ of generalized data, then we get a generalized datum for $G$.

(iii) The generalized datum $(\vec{G}, y, \vec{r}, \rho, \vec{\phi})$ is generic if and only if $\left(\vec{G}_{j}, y_{j}, \vec{r}, \rho^{(j)}, \vec{\phi}^{(j)}\right)$ is generic for each $j$.

(iv) The supercuspidal representation of $G(F)$ constructed from $(\vec{G}, y, \vec{r}, \rho, \vec{\phi})$ is the tensor product of the supercuspidal representations of $G_{j}(F)$ constructed from $\left(\vec{G}_{j}, y_{j}, \vec{r}, \rho^{(j)}, \vec{\phi}^{(j)}\right)$.

(v) A supercuspidal representation $\pi=\bigotimes \pi_{j}$ of $G(F)=\prod G_{j}(F)$ arises from our construction if and only if each $\pi_{j}$ does.

\section{A lemma ABout double Cosets}

Let $\left(G^{\prime}, G\right)$ be a tamely ramified twisted Levi sequence, $y \in \mathcal{B}\left(G^{\prime}, F\right) \subset \mathcal{B}(G, F)$. Let $r, s$ be real numbers such that $s \geq r / 2>0$. Put $J=\left(G^{\prime}, G\right)(F)_{y,(r, s)}$ and $J^{\prime}=G^{\prime}(F)_{y, r}=J \cap G^{\prime}(F)$.

Special cases of the following lemma have been proved in the works of Howe and Moy. The proof here follows their ideas. The new feature is that we have to use two tori, and instead of using the Iwahori decomposition, we use the decomposition in $\$ 13$

Lemma 16.1. For any $g \in G^{\prime}(F), J g J \cap G^{\prime}(F)=J^{\prime} g J^{\prime}$.

Corollary 16.2. The map

$$
J^{\prime} \backslash G^{\prime}(F) / J^{\prime} \rightarrow J \backslash\left(J G^{\prime}(F) J\right) / J, \quad J^{\prime} g J^{\prime} \mapsto J g J
$$

is a well-defined bijection.

Proof. Let $T$ be a maximal torus of $G^{\prime}$ such that $T$ is split over a tamely ramified Galois extension $E / F$, and let $y, g . y \in A\left(G^{\prime}, T, F\right)$. We form the groups $J_{1}(E), J_{2}(E), J_{3}(E)$ as in $\$ 13$ such that $J(E)=J_{1}(E) J_{2}(E) J_{3}(E)$. (In $\sqrt{13}$ we assume $s=r / 2$. But that hypothesis can be weakened to $s \geq r / 2$.)

Replace $(y, g, T)$ by $\left(y^{\bullet}, g^{\bullet}, T^{\bullet}\right)=\left(y, g^{-1}, g^{-1} T\right)$ (observe that $\left(y^{\bullet}, g^{\bullet} \cdot y^{\bullet}\right)=$ $g^{\bullet}$.(g.y,y) is a pair of elements lying on $\left.A\left(G^{\prime}, T^{\bullet}, F\right)\right)$. We form another decomposition $J(E)=J_{1}^{\bullet}(E) J_{2}^{\bullet}(E) J_{3}^{\bullet}(E)$.

We now compare these groups. The root system $\Phi^{\bullet}=\Phi\left(G, T^{\bullet}, E\right)$ is simply $\{\operatorname{Ad}(g) . a: a \in \Phi=\Phi(G, T, E)\}$, where $\operatorname{Ad}(g) . a: g^{\bullet} T \rightarrow \mathbb{G}_{\mathrm{m}}$ is $t \mapsto a\left(\left(g^{\bullet}\right)^{-1} t g^{\bullet}\right)$. For $a \in \Phi$, we have ${ }^{g^{\bullet}} G_{a}(E)_{y, t}=G_{\operatorname{Ad}\left(g^{\bullet}\right) . a}(E)_{g^{\bullet} \cdot y, t}$ for any $t \in \tilde{\mathbb{R}}$. We also have $a(y-g \cdot y)=\operatorname{Ad}\left(g^{\bullet}\right) \cdot a\left(g^{\bullet} \cdot y-y\right)=-\operatorname{Ad}\left(g^{\bullet}\right) \cdot a\left(y^{\bullet}-g^{\bullet} \cdot y^{\bullet}\right)$. Therefore, if $a \in \Phi_{i}$, then $\operatorname{Ad}\left(g^{\bullet}\right) . a \in \Phi_{4-i}^{\bullet}$. 
Let $f$ (resp. $\left.f^{\bullet}\right)$ be the concave function on $\Phi(G, T, E)\left(\operatorname{resp} . \Phi\left(G, T^{\bullet}, E\right)\right)$ such that $J(E)=G(E)_{y, f}\left(\right.$ resp. $\left.J(E)=G(E)_{y, f} \bullet\right)$. Then

$$
\begin{aligned}
g^{\bullet} G_{a}(E)_{y, f(a)} & =G_{\operatorname{Ad}\left(g^{\bullet}\right) . a}(E)_{g} \bullet \cdot y, f(a) \\
& =G_{\operatorname{Ad}\left(g^{\bullet}\right) . a}(E)_{y, f(a)+\operatorname{Ad}\left(g^{\bullet}\right) . a(y-g \bullet . y)}=G_{\operatorname{Ad}\left(g^{\bullet}\right) . a}(E)_{y, f(a)-a(y-g . y)} .
\end{aligned}
$$

Since $f(a)=f^{\bullet}\left(\operatorname{Ad}\left(g^{\bullet}\right) . a\right)$, we conclude that $g^{\bullet} J_{1}(E) \subset J_{3}^{\bullet}(E), g^{\bullet} J_{2}(E)=J_{2}^{\bullet}(E)$, and $g^{\bullet} J_{3}(E) \supset J_{1}^{\bullet}(E)$.

Of course, this implies that ${ }^{g} J_{3}^{\bullet} \supset J_{1},{ }^{g} J_{2}^{\bullet}=J_{2}$, and ${ }^{g} J_{1}^{\bullet} \subset J_{3}$.

Now we have $J g J=J_{3} J_{2} J_{1} g J=J_{3} g\left(g^{-1} J_{2} J_{1} g\right) J=J_{3} g J=J_{3} g J_{1}^{\bullet} J_{2}^{\bullet} J_{3}^{\bullet}=$ $J_{3}\left(g J_{1}^{\bullet} g^{-1}\right) g J_{2}^{\bullet} J_{3}^{\bullet}=J_{3} g J_{2}^{\bullet} J_{3}^{\bullet}$.

So for any $x \in J g J$, we can write $x=j g k$, where $j \in J_{3}, k \in J_{2}^{\bullet} J_{3}^{\bullet}$. We claim that $j$ and $k$ are unique. Indeed, if $j g k=j^{*} g k^{*}$, then $\left(j^{*}\right)^{-1} j=g k^{*}\left(k^{-1}\right) g^{-1} \in$ $J_{3} \cap g\left(J_{2}^{\bullet} J_{3}^{\bullet}\right) g^{-1} \subset G_{\Phi_{3}}(E) \cap G_{\Phi_{1} \cup \Phi_{2} \cup\{0\}}(E)=\{1\}$. Therefore, $j=j^{*}, k=k^{*}$. Here we are writing $G_{\Psi}(E)$ for the subgroup generated by $G_{a}(E)$ for all $a \in \Psi \subset \Phi \cup\{0\}$.

Similarly, we have a unique decomposition $J g J=J_{3} J_{2} g J_{3}^{\bullet}$. But we shall not need this.

Choose $z \in X_{*}(T, E) \otimes \mathbb{R}$ such that $z$ lies in a Weyl chamber whose closure contains $y-g . y$. Then $\Phi^{+}=\{a \in \Phi: a(z)>0\}$ is the system of positive roots determined by $z$. Then $\Phi_{3} \subset \Phi_{+}, \Phi_{1} \subset \Phi^{-}=-\Phi^{+}$. Let $\Phi^{\prime}=\Phi\left(G^{\prime}, T, E\right)$.

Lemma 16.3. Let $\left(g_{a}\right)_{a \in \Phi^{0}}$ be a family of elements such that $g_{a} \in G_{a}(E)$ for each $a \in \Phi^{0}$. Suppose that

$$
x=\left(\prod_{a \in \Phi^{+}} g_{a}\right) g_{0}\left(\prod_{a \in \Phi^{-}} g_{a}\right)
$$

is an element of $G^{\prime}(E)$, where the product is taken in any fixed order. Then $g_{a}=1$ for $a \notin \Phi^{\prime} \cup\{0\}$.

Proof. We first rephrase (using $[\mathrm{Hu}, 28.1])$ : let $U_{ \pm}=\prod_{a \in \Phi^{ \pm}} G_{a}$; if $x \in G^{\prime}(E)$ has a "big cell" decomposition $x=u_{+} g_{0} u_{-}$, then $u_{ \pm} \in U_{ \pm}^{\prime}(E)=U_{ \pm}(E) \cap G^{\prime}(E)$ (and therefore $x$ is in the big cell of $G^{\prime}(E)$ ).

Write $u_{+}=u_{+}^{\prime \prime} u_{+}^{\prime}$, where $u_{+}^{\prime \prime} \in U_{+}^{\prime \prime}(E)=\prod_{a \in \Phi^{+} \backslash \Phi^{\prime}} G_{a}(E), u_{+}^{\prime} \in U_{+}^{\prime}(E)$. Similarly write $u_{-}=u_{-}^{\prime} u_{-}^{\prime \prime}$. Then $x=\left(u_{+}^{\prime \prime}\right)\left(u_{+}^{\prime} g_{0} u_{-}^{\prime}\right)\left(u_{-}^{\prime \prime}\right)$. But the multiplication $\operatorname{map} U_{+}^{\prime \prime}(E) \times G^{\prime}(E) \times U_{-}^{\prime \prime}(E) \rightarrow G(E)$ is injective. Therefore, $u_{ \pm}^{\prime \prime}=1$ and $u_{ \pm} \in$ $U_{ \pm}^{\prime}(E)$.

Now we return to the proof of Lemma 16.1. If $x=j_{3} g j_{2}^{\bullet} j_{3}^{\bullet} \in J_{3} g J_{2}^{\bullet} J_{3}^{\bullet}=J g J$ lies in $G^{\prime}(F)$, then $x g^{-1}=j_{3}\left(g j_{2}^{\bullet} g^{-1}\right)\left(g j_{3}^{\bullet} g^{-1}\right) \in J_{3} J_{2} G_{\Phi_{1}}(E)$. By [BT1, 6.4.48] and Lemma 16.3 this implies that $j_{3} \in J_{3} \cap G^{\prime}(F) \subset J^{\prime}$, and $j_{2}^{\bullet} \in J_{2}^{\bullet} \cap G^{\prime}(F) \subset J^{\prime}$, $j_{3}^{\bullet} \in J_{3}^{\bullet} \cap G^{\prime}(F) \subset J^{\prime}$. Therefore, $x \in J^{\prime} g J^{\prime}$. The lemma is proved.

\section{A Hecke Algebra isomorphism}

We now return to the setting of 914 Let $\left(G^{\prime}, G\right)$ be a tamely ramified twisted Levi sequence, and let $y \in \mathcal{B}\left(G^{\prime}, F\right) \subset \mathcal{B}(G, F)$. Let $\phi$ be a $G$-generic character of $G^{\prime}(F)$ relative to $y$ of depth $r$.

Recall that $J=\left(G^{\prime}, G\right)_{y,(r, r / 2)}, J_{+}=\left(G^{\prime}, G\right)_{y,(r,(r / 2)+)}$. We also put $J^{\prime}=$ $G^{\prime}(F)_{y, r}=J \cap G^{\prime}(F)$.

Our notation of Hecke algebra is that of [BK1, 4.1]. We find it convenient to write $\check{\mathcal{H}}(G, \rho)$ for $\mathcal{H}(G, \check{\rho})$, where $\check{\rho}$ is the contragradient of $\rho$. 
Theorem 17.1. If $G^{\prime}$ is a Levi factor of a parabolic subgroup $P$ of $G$, then there exists a support-preserving algebra isomorphism

$$
\check{\mathcal{H}}(G(F), \tilde{\phi}) \simeq \check{\mathscr{H}}\left(G^{\prime}(F),\left.\phi\right|_{J^{\prime}}\right) .
$$

Proof. Following a trick of Howe and Moy, we define a group $J_{\vdash}$ between $J$ and $J_{+}$as follows: Choose a maximal torus $T$ of $G^{\prime}$ such that $T$ is split over a tamely ramified extension $E$, and $y \in A(G, T, E)$. Let $U^{+}$be the unipotent radical of $P$, and let $U^{-}$be the unipotent subgroup "opposite to" $U^{+}$. Define

$$
f(a)= \begin{cases}r & \text { if } a \in \Phi\left(G^{\prime}, T, E\right) \cup\{0\}, \\ (r / 2)+ & \text { if } a \in \Phi\left(U^{+}, T, E\right), \\ (r / 2) & \text { if } a \in \Phi\left(U^{-}, T, E\right),\end{cases}
$$

and $J_{\vdash}=G(F)_{y, f}$. By BT1 6.4.44], $\left[J_{\vdash}, J_{\vdash}\right] \subset\left(G^{\prime}, G\right)(F)_{y,(r+, s+)} \subset \operatorname{ker} \hat{\phi}$. There is a unique character of $J_{\vdash} /\left(G^{\prime}, G\right)(F)_{y,(r+, s+)}$ which is realized by the $G$-generic element $X^{*}$ realizing $\left.\phi\right|_{G(F)_{y, r}: r+}$. We denote this character by $\phi_{\vdash}$. By a well-known fact about Heisenberg representations,

$$
\operatorname{ind}_{J_{\vdash}}^{J} \phi_{\vdash} \simeq \tilde{\phi}
$$

and therefore, $\check{\mathcal{H}}(G(F), \tilde{\phi}) \simeq \check{\mathcal{H}}\left(G(F), \phi_{\vdash}\right)$ by BK1, 4.1.3]. It suffices to show that there is a support-preserving isomorphism $\check{\mathcal{H}}\left(G(F), \phi_{\vdash}\right) \simeq \check{\mathcal{H}}\left(G^{\prime}(F),\left.\phi\right|_{J^{\prime}}\right)$.

Lemma 17.2. The support of $\check{\mathcal{H}}\left(G(F), \phi_{\vdash}\right)$ is exactly $J_{\vdash} G^{\prime}(F) J_{\vdash}$.

Proof. Notice that by Theorem 9.4 the support is contained in $J G^{\prime}(F) J$, and by Proposition [12.3 and [BK1, 4.1.5], the subspace of $\check{\mathcal{H}}\left(G(F), \phi_{\vdash}\right)$ consisting of functions with support on $J g J$ is exactly 1-dimensional, for all $g \in G^{\prime}(F)$. To prove the lemma, it suffices to show that the unique (up to a scalar multiple) non-zero function in this space is supported on $J_{\vdash} g J_{\vdash}$. In other words, it suffices to show that every $g \in G^{\prime}(F)$ intertwines $\phi_{\vdash}$.

The last statement can be proved by the method of Lemma 9.3 with obvious modifications.

Now we are ready to apply $\mathrm{BK2}$, Theorem 7.2 (ii)]. Indeed, the conditions in BK2, 6.1] are obviously satisfied. By the lemma we just proved, $\check{\mathcal{H}}\left(J_{\vdash} G^{\prime}(F) J_{\vdash}, \phi_{\vdash}\right)$ is the whole of $\check{\mathcal{H}}\left(G(F), \phi_{\vdash}\right)$, hence is certainly a subalgebra of $\check{\mathcal{H}}\left(G(F), \phi_{\vdash}\right)$. Thus all hypotheses of [BK2, Theorem 7.2 (ii)] are satisfied and the theorem is proved completely.

Corollary 17.3. Suppose that $G^{\prime}$ is a Levi factor of a proper parabolic subgroup $P$ of $G$. If $\pi$ is an irreducible admissible representation of $G(F)$ such that $\pi$ contains the minimal $K$-type $\left(G(F)_{y, r}, \hat{\phi}\right)$, then $\pi$ is not supercuspidal.

Proof. By $[\overline{\mathrm{Ad}}, 2.3 .4], \pi$ contains $\left(J_{+}, \hat{\phi}\right)$, and hence also $(J, \tilde{\phi})$.

Since $Z\left(G^{\prime}\right)(F) / Z(G)(F)$ is not compact, $\pi$ is not supercuspidal by a well-known argument.

Remark 17.4. The isomorphism constructed in the proof of Theorem 17.1 depends on the parabolic subgroup $P$ in general.

Remark 17.5. The preceding theorem and its corollary are true in any residual characteristic (i.e. residual characteristic 2 is not a problem). This is because the 
whole proof concerns only the Heisenberg representations (but not the Weil representations), and Heisenberg representations can be easily done in a more general setting.

Remark 17.6. This Hecke algebra isomorphism contains a large part of Howe-Moy's Hecke algebra isomorphism for "separated minimal $K$-types of $\mathrm{GL}_{n}$ ".

Conjecture 17.7. Using the setting in the beginning of this section, there exists a support-preserving algebra isomorphism

$$
\check{\mathcal{H}}(G(F), \tilde{\phi}) \simeq \check{\mathcal{H}}\left(G^{\prime}(F),\left.\phi\right|_{J^{\prime}}\right) .
$$

Remark 17.8. This Hecke algebra isomorphism will imply that there is a bijection from

\{irreducible admissible representations of $G^{\prime}(F)$ containing $\left.\left.\phi\right|_{J^{\prime}}\right\}$

to

\{irreducible admissible representations of $G(F)$ containing $\tilde{\phi}$ \}

by [BK1, 4.2.3]. With the hypothesis of Theorem[17.1, this bijection is essentially a parabolic induction/Jaquet functor $([\mathrm{BK} 2, \S 12])$. This bijection should send $\pi_{i-1}$ to $\pi_{i}$ when applied to the pair $\left(G_{i-1}, G_{i}\right)$ in the set-up of Theorem 15.1

As evidence for this conjecture, we have: (i) it is true when $G^{\prime}$ is a Levi factor of a parabolic subgroup over $F$ (by the above theorem); (ii) it is true when $G^{\prime} / Z(G)$ is anisotropic (this is essentially in [Ad], but we will give a proof below for completeness); (iii) it is true in many cases when $G=\mathrm{GL}_{n}$ with $(n, p)=1$ (by $[\mathrm{HM}]$ ); (iv) we have the following partial result:

Theorem 17.9. There exists a support-preserving vector space isomorphism

$$
\check{\mathcal{H}}(G(F), \tilde{\phi}) \simeq \check{\mathcal{H}}\left(G^{\prime}(F),\left.\phi\right|_{J^{\prime}}\right) .
$$

Proof. If $\rho$ is a representation of $K$ and $X \subset G$ is such that $K X K=X$, we use $\mathscr{\mathcal { H }}(X, \rho)$ to denote the subspace of $\check{\mathcal{H}}(G, \rho)$ consisting of those functions whose supports lie in $X$.

By Theorem 9.4 and Proposition $12.3 \tilde{\mathcal{H}}(G(F), \tilde{\phi})$ is the direct sum of 1-dimensional subspaces:

$$
\check{\mathcal{H}}(G(F), \tilde{\phi})=\bigoplus_{X \in J \backslash J G^{\prime}(F) J / J} \check{\mathcal{H}}(X, \tilde{\phi}) .
$$

By Lemma 9.3. $\check{\mathcal{H}}\left(G(F),\left.\phi\right|_{J^{\prime}}\right)$ is also a direct sum of 1-dimensional subspaces:

$$
\check{\mathcal{H}}\left(G^{\prime}(F),\left.\phi\right|_{J^{\prime}}\right)=\bigoplus_{X^{\prime} \in J^{\prime} \backslash G^{\prime}(F) / J^{\prime}} \check{\mathcal{H}}\left(X^{\prime},\left.\phi\right|_{J^{\prime}}\right) .
$$

By choosing a linear isomorphism

$$
\check{\mathcal{H}}\left(J^{\prime} g J^{\prime},\left.\phi\right|_{J^{\prime}}\right) \rightarrow \check{\mathcal{H}}(J g J, \tilde{\phi})
$$

for each $g \in J^{\prime} \backslash G^{\prime}(F) / J^{\prime}$ and applying Corollary 16.2, we obtain a vector space isomorphism $\check{\mathcal{H}}\left(G^{\prime}(F),\left.\phi\right|_{J^{\prime}}\right) \simeq \check{\mathcal{H}}(G(F), \tilde{\phi})$ and the theorem is proved.

The following lemma shows that a natural isomorphism exists between certain subalgebras of the two Hecke algebras. 
Lemma 17.10. Let $K=G^{\prime}(F) \cap G(F)_{[y]}$. There is a natural support-preserving algebra isomorphism from $\check{\mathcal{H}}(K J, \tilde{\phi})$ to $\check{\mathcal{H}}\left(K J^{\prime},\left.\phi\right|_{J^{\prime}}\right)$.

Proof. There is a Weil representation $\phi^{\prime}: K J \rightarrow \mathrm{GL}(W)$ extending the Heisenberg representation $\tilde{\phi}$ of $J$. For any $g \in K$, let $f_{g}$ be the function from $K J$ to $\operatorname{End}(W)$ such that $f_{g}$ is supported on $J g J=g J$ and $\left.f_{g}\right|_{g J}=\left.\phi^{\prime}\right|_{g J}$. Similarly let $f_{g}^{\prime}$ be the function from $K=K J^{\prime}$ to $\mathbb{C}$ such that $f_{g}^{\prime}$ is supported on $J^{\prime} g J^{\prime}=g J^{\prime}$ and $\left.f_{g}^{\prime}\right|_{g J^{\prime}}=\left.\phi\right|_{g J^{\prime}}$.

It is clear that $\left\{f_{g}^{\prime}\right\}_{g \in K}$ spans $\check{\mathcal{H}}\left(K J^{\prime},\left.\phi\right|_{J^{\prime}}\right)$ and $\left\{f_{g}\right\}_{g \in K}$ spans $\check{\mathcal{H}}(K J, \tilde{\phi})$. There is a unique vector space isomorphism $\eta: \check{\mathcal{H}}\left(K J^{\prime},\left.\phi\right|_{J^{\prime}}\right) \rightarrow \check{\mathcal{H}}(K J, \tilde{\phi})$ such that $\eta\left(f_{g}^{\prime}\right)=f_{g}$.

It is immediately verified that for any $g, h \in K$, we have (i) $f_{g}^{\prime} * f_{h}^{\prime}=f_{g h}^{\prime}$ and (ii) $f_{g} * f_{h}=f_{g h}$. Therefore, $\eta$ is an algebra isomorphism.

Corollary 17.11. If $G^{\prime} / Z(G)$ is anisotropic, then $\check{\mathcal{H}}(G(F), \tilde{\phi}) \simeq \check{\mathcal{H}}\left(G^{\prime}(F),\left.\phi\right|_{J^{\prime}}\right)$.

\section{REFERENCES}

[Ad] J.D. Adler: Refined anisotropic K-types and supercuspidal representations, Pacific J. Math. 185, no. 1, 1-32 (1998) MR 2000f:22019

[AR] J.D. Adler and A. Roche: An intertwining result for p-adic groups, Canad. J. Math. 52, no. 3, 449-467 (2000) CMP 2000:12

[BK1] C.J. Bushnell and P.C. Kutzko: The admissible dual of GL(N) via compact open subgroups, Annals Math. Studies, Princeton Univ. Press (1993) MR 94h:22007

[BK2] C.J. Bushnell and P.C. Kutzko: Smooth representations of reductive p-adic groups: structure theory via types, Proc. London Math. Soc. 77, no. 3, 582-634 (1998) MR 2000c:22014

[BT1] F. Bruhat et J. Tits: Groupes réductifs sur un corps local, Chapitre I, Publ. Math. I.H.E.S. 41, 5-251 (1972) MR 48:6265

[BT2] F. Bruhat et J. Tits: Groupes réductifs sur un corps local, Chapitre II, Publ. Math. I.H.E.S. 60, 197-376 (1984) MR 86c:20042

[Co] L. Corwin, A construction of the supercuspidal representations of $\mathrm{GL}_{n}(F), F$-adic, Trans. Amer. Math. Soc. 337, 1-58 (1993) MR 93g:22017

[De] S. DeBacker, On supercuspidal characters of $\mathrm{GL}_{\ell}, \ell$ a prime, $\mathrm{Ph}$. D. thesis, The University of Chicago (1997)

[Ge] P. Gérardin: Weil representations associated to finite fields, J. Algebra 46, 54-101 (1977) MR 57:470

[Ho] R. Howe: Tamely ramified supercuspidal representations for $\mathrm{GL}_{n}$, Pacific. J. Math. 73, 437-460 (1977) MR 58:11241

$[\mathrm{HM}] \quad$ R. Howe and A. Moy: Hecke algebra isomorphisms for $\mathrm{GL}_{n}$ over a p-adic field, J. Algebra 131, 388-424 (1990) MR 91f:22031

[Hu] J.E. Humphreys: Linear algebraic groups, Springer-Verlag (1975) MR 53:633

[Ki] J. Kim, Hecke algebras of classical groups over p-adic fields and supercuspidal representations, Amer. J. Math. 121, 967-1029 (1999) CMP 2000:01

[KZ] H. Koch and E.-W. Zink: Zur Korrespondenz von Darstellungen der Galoisgruppen und der zentralen Divisionsalgebren über lokalen Körpern (Der Zahme Fall), Math. Nachr. 98, 83-119 (1980) MR 83h:12025

[L] George Lusztig: Classification of unipotent representations of simple p-adic groups, Internat. Math. Res. Notices 11, 517-589 (1995) MR 98b:22034

[Mo1] L.E. Morris, Level zero G-types, Comp. Math. 118, 135-157 (1999) MR 2000g:22029

[Mo2] L.E. Morris, Some tamely ramified supercuspidal representations of symplectic groups, Proc. London Math. Soc. 63, 519-551 (1990) MR 92i:22017

[Mo3] L.E. Morris, Tamely ramified supercuspidal representations of classical groups I, II, Ann. Sci. Éc. Norm. Sup. 24, 705-738 (1991), 25, 233-274 (1992) MR 93c:22032 MR 93h:22032 
[Mo4] L.E. Morris, Tamely ramified intertwining algebras, Invent. Math. 114, 1-54 (1993) MR 94g:22035

[My] A. Moy: Local constants and the tame Langlands correspondence, Amer. J. Math. 108, 863-930 (1986) MR 88b:11081

[MP1] A. Moy and G. Prasad: Unrefined minimal K-types for p-adic groups, Inv. Math. 116, 393-408 (1994) MR 95f:22023

[MP2] A. Moy and G. Prasad: Jaquet functors and unrefined minimal K-types, Comment. Math. Helvetici 71, 96-121 (1996) MR 97c:22021

[P] G. Prasad, Galois-fixed points in the Bruhat-Tits building of a reductive group, Bulletin Soc. Math. France, to appear.

[Se] J.-P. Serre: Linear representations of finite groups, Springer-Verlag (1977) MR 56:8675

[Sp] T.A. Springer: Reductive groups, Automorphic forms, representations, and $L$-functions (A. Borel and W. Casselman, Eds.), v.1., 3-28 (1977) MR 80h:20062

[St] R. Steinberg: Torsion in reductive groups, Adv. in Math. 15, 63-92 (1975) MR 50:7369

[Ti] J. Tits: Reductive groups over local fields, Automorphic forms, representations, and $L$ functions (A. Borel and W. Casselman, Eds.), v.1., 29-69 (1977) MR 80h:20064

Department of Mathematics, Princeton University, Princeton, New Jersey 08540

E-mail address: yu@math.princeton.edu

Current address: Department of Mathematics, University of Maryland, College Park, Maryland 20742

E-mail address: yu@math.umd.edu 